\title{
Pharmacokinetics and Metabolites of Twelve Bioactive \\ Polymethoxyflavones in Rat Plasma
}

3,5,7,4'-Tetramethoxyflavone (1)

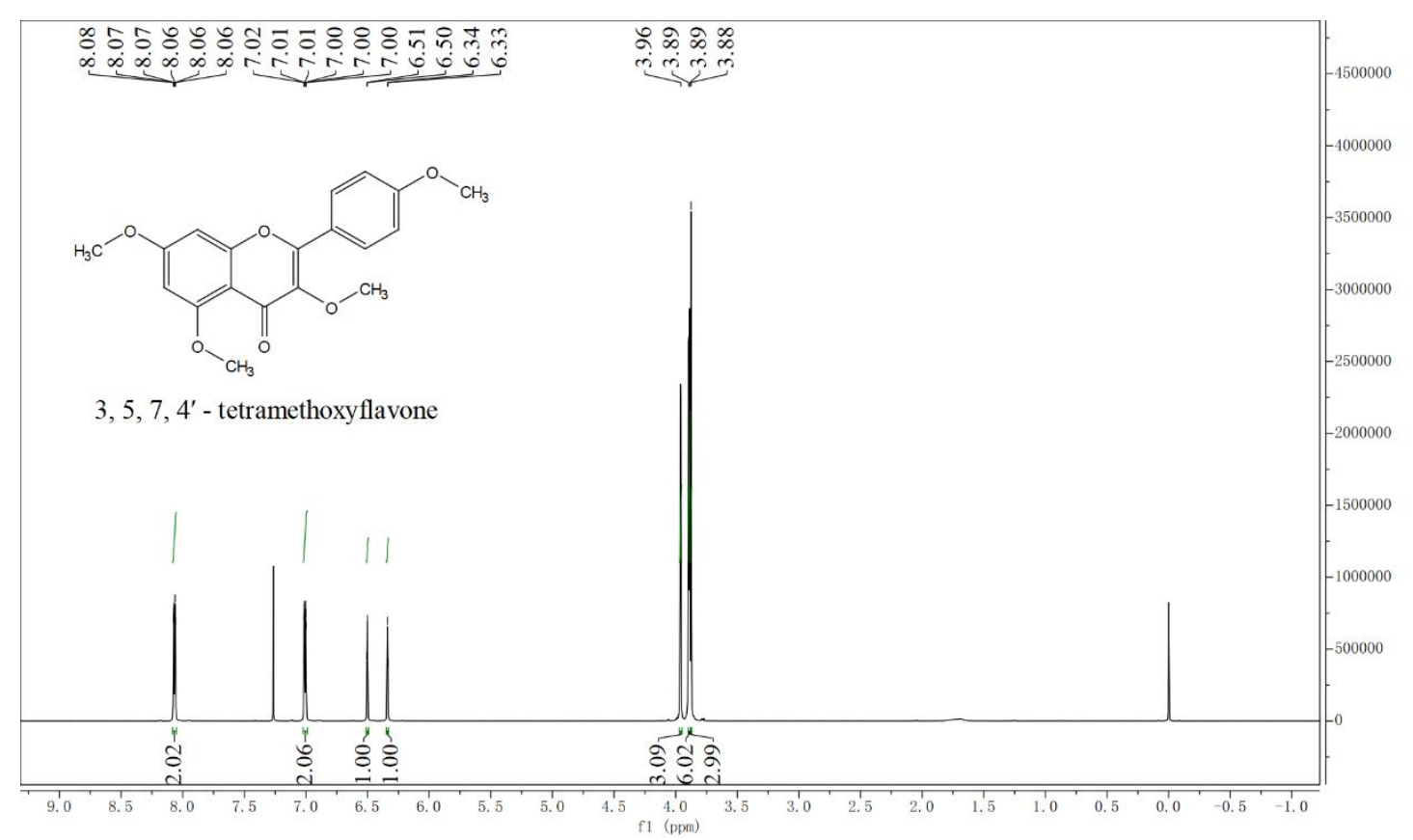

5,6,7,4'-Tetramethylflavone (2) 


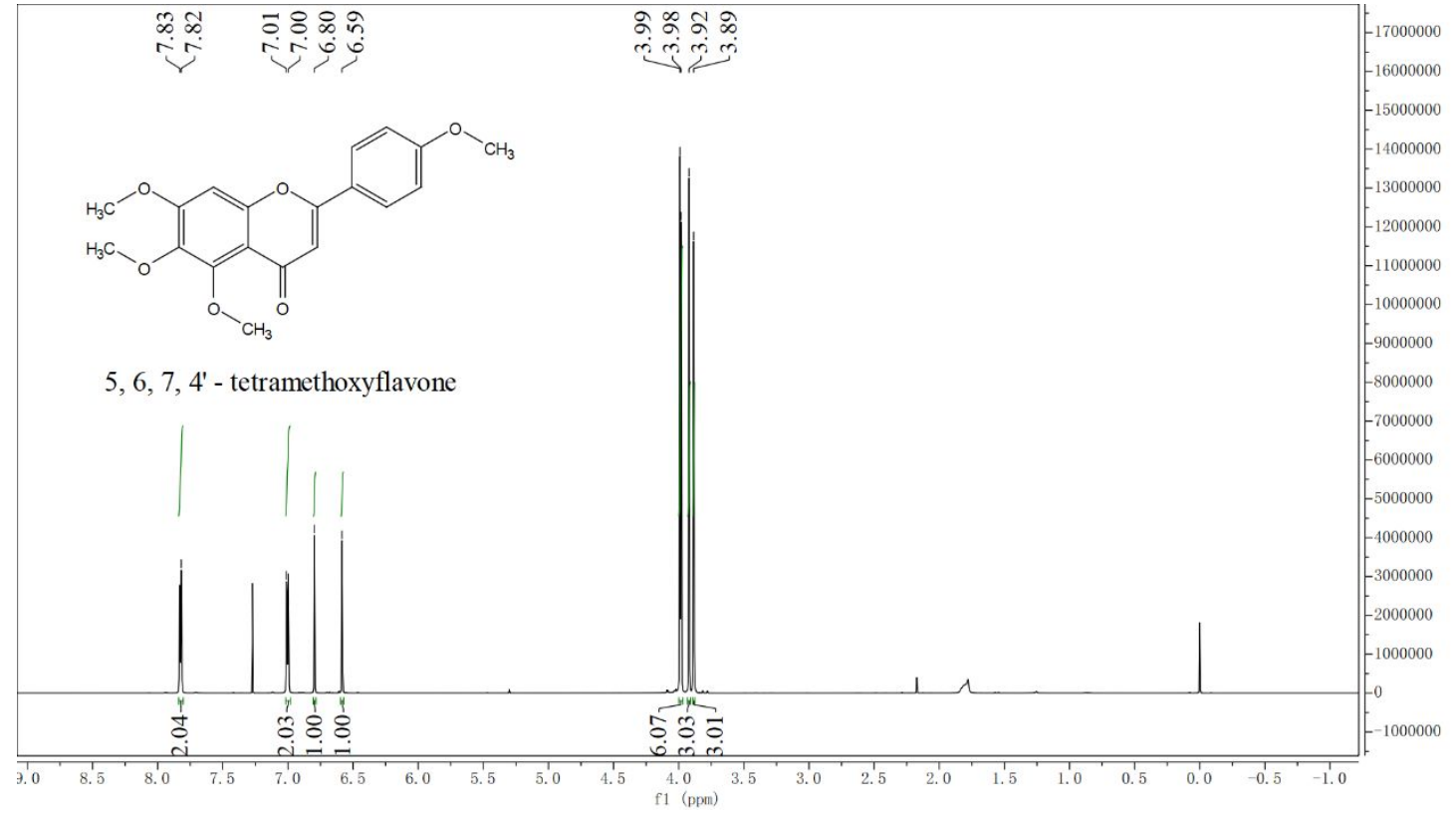

3,7,3',4'-Tetramethoxyflavone (3)

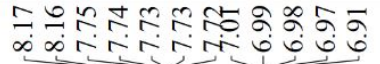

कूळळం

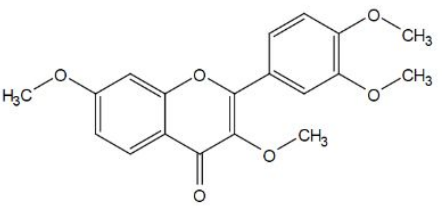

3, 7, 3', 4' - tetramethoxyflavone

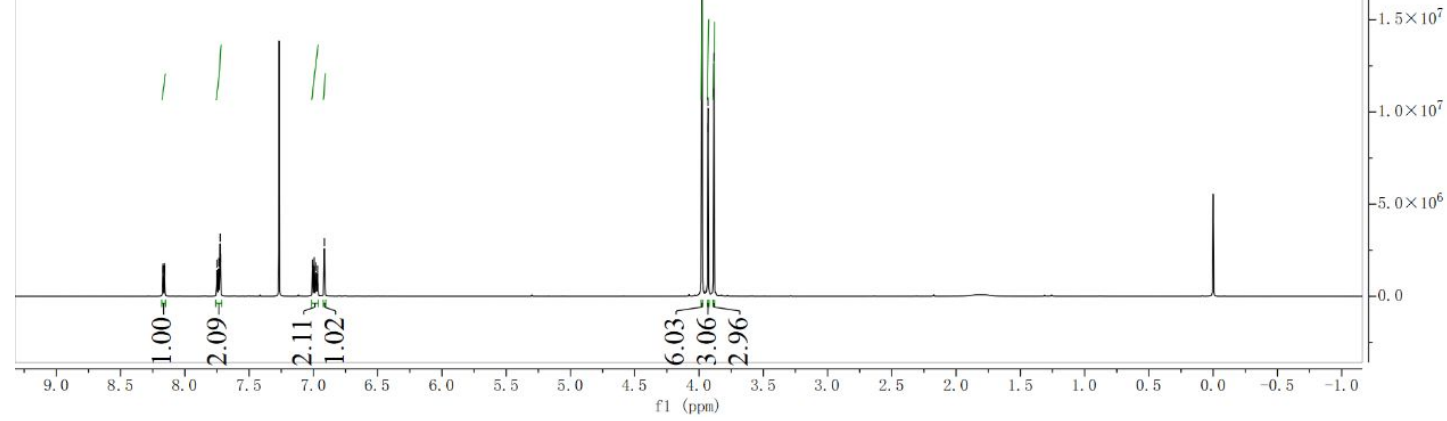

$5,7,3^{\prime}, 4^{\prime}$-Tetramethoxyflavone (4) 


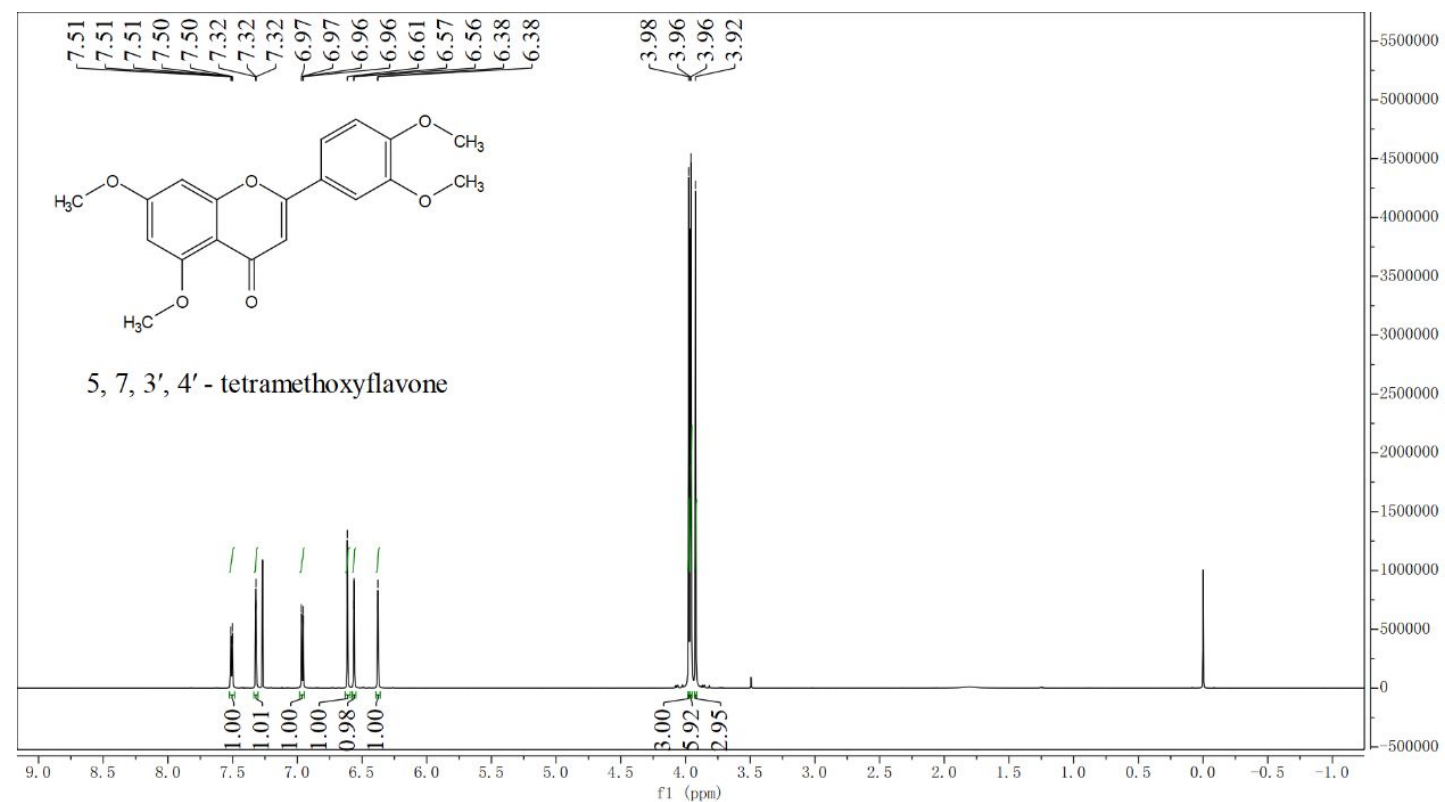

5-Hydroxy-3,7,2',4'-Tetramethoxyflavone (5)

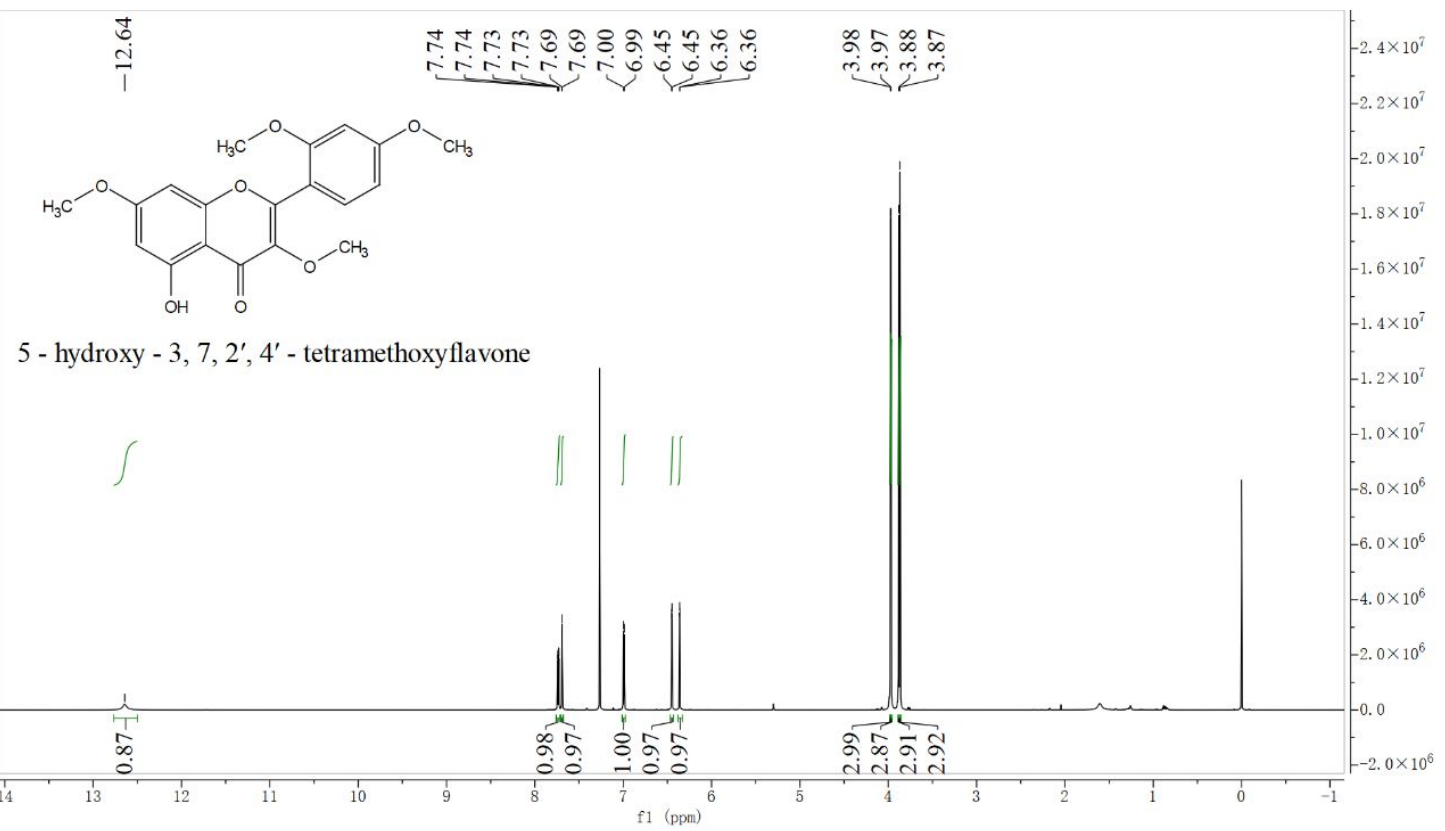

3,5,7,2',4'-Pentamethoxyflavone (6) 


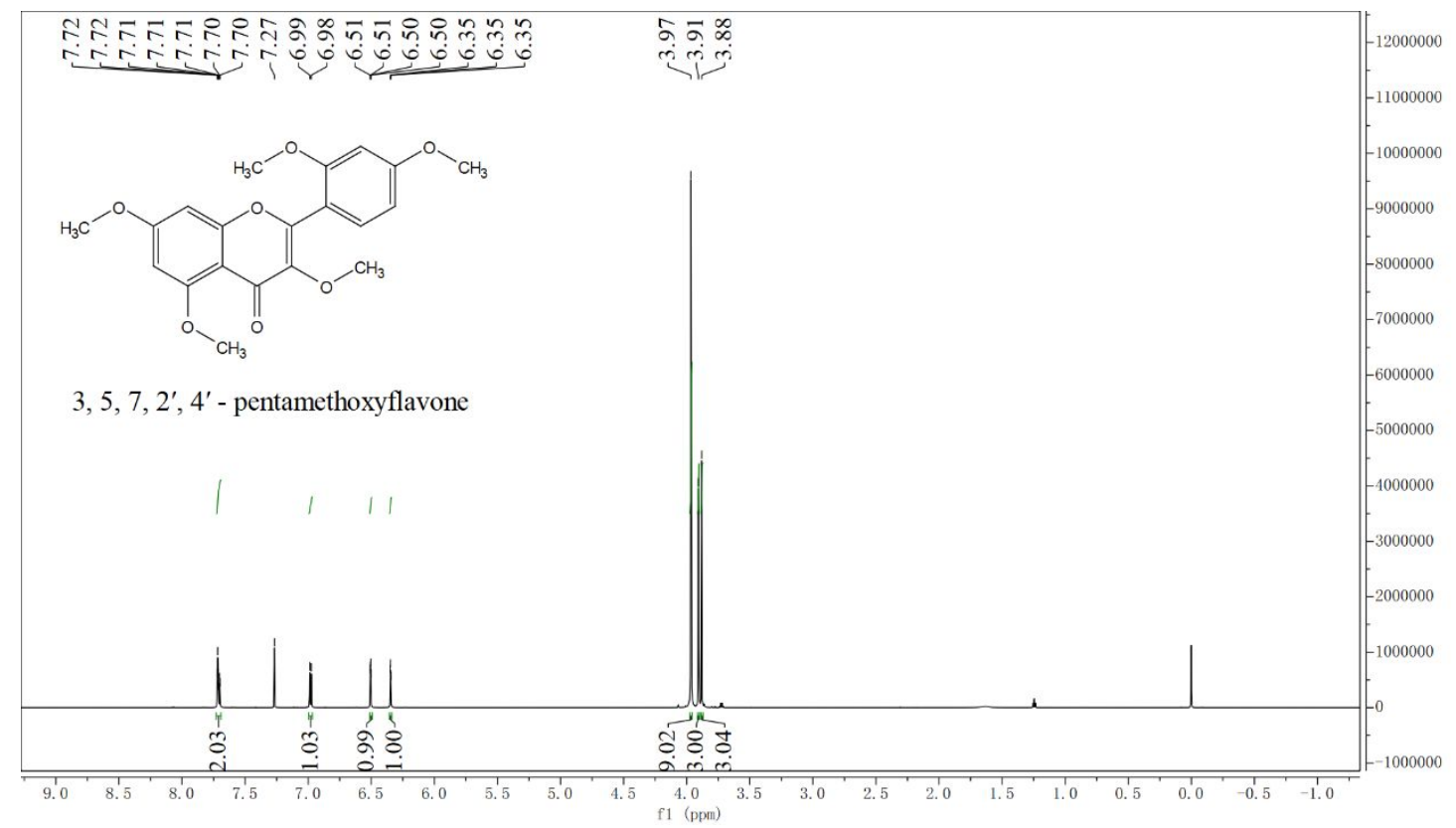

5- Hydroxy-3, 7,3',4'-Tetramethoxyflavone (7)

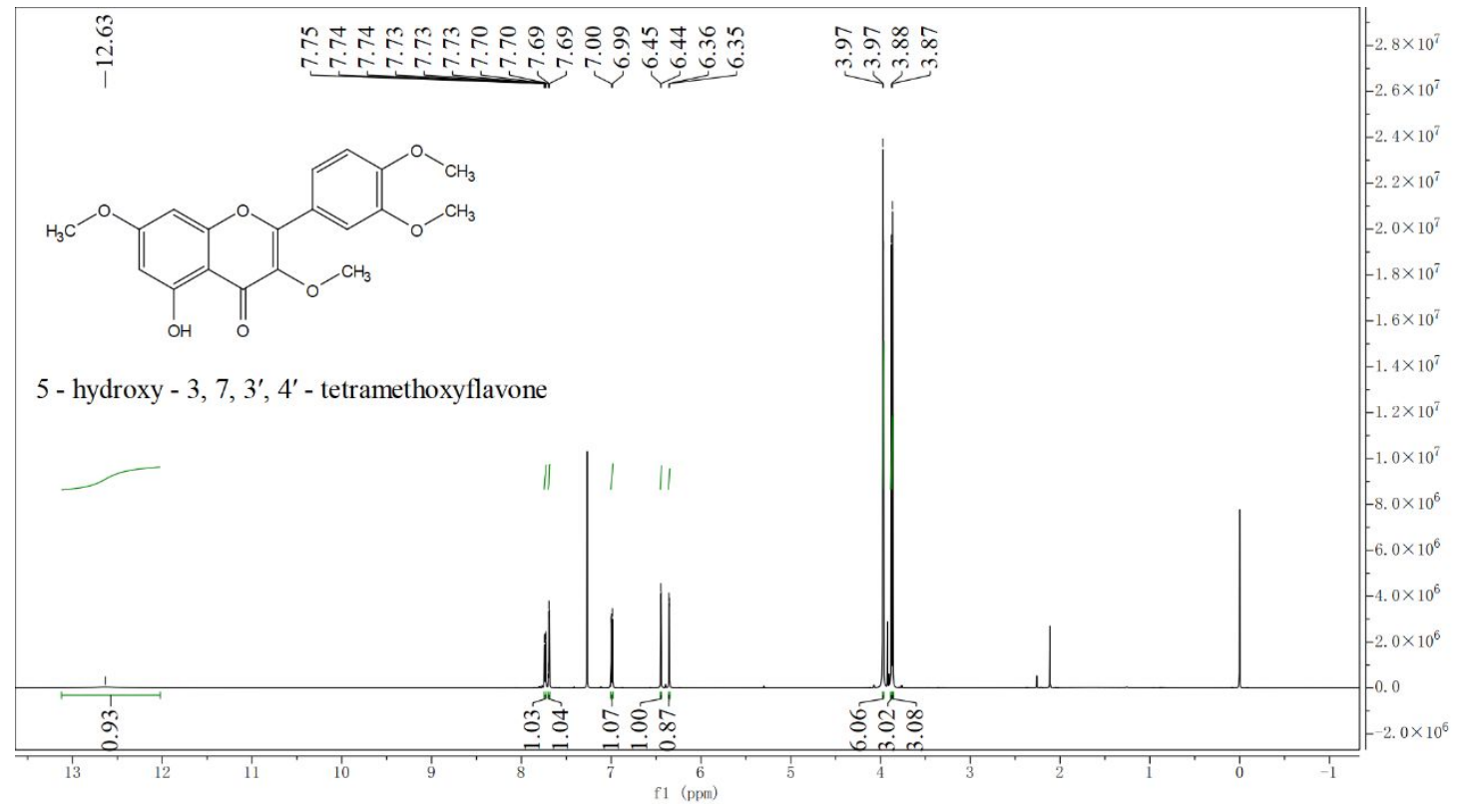

3-Hydroxy-5, 7,3',4'-Tetramethylflavone (8) 


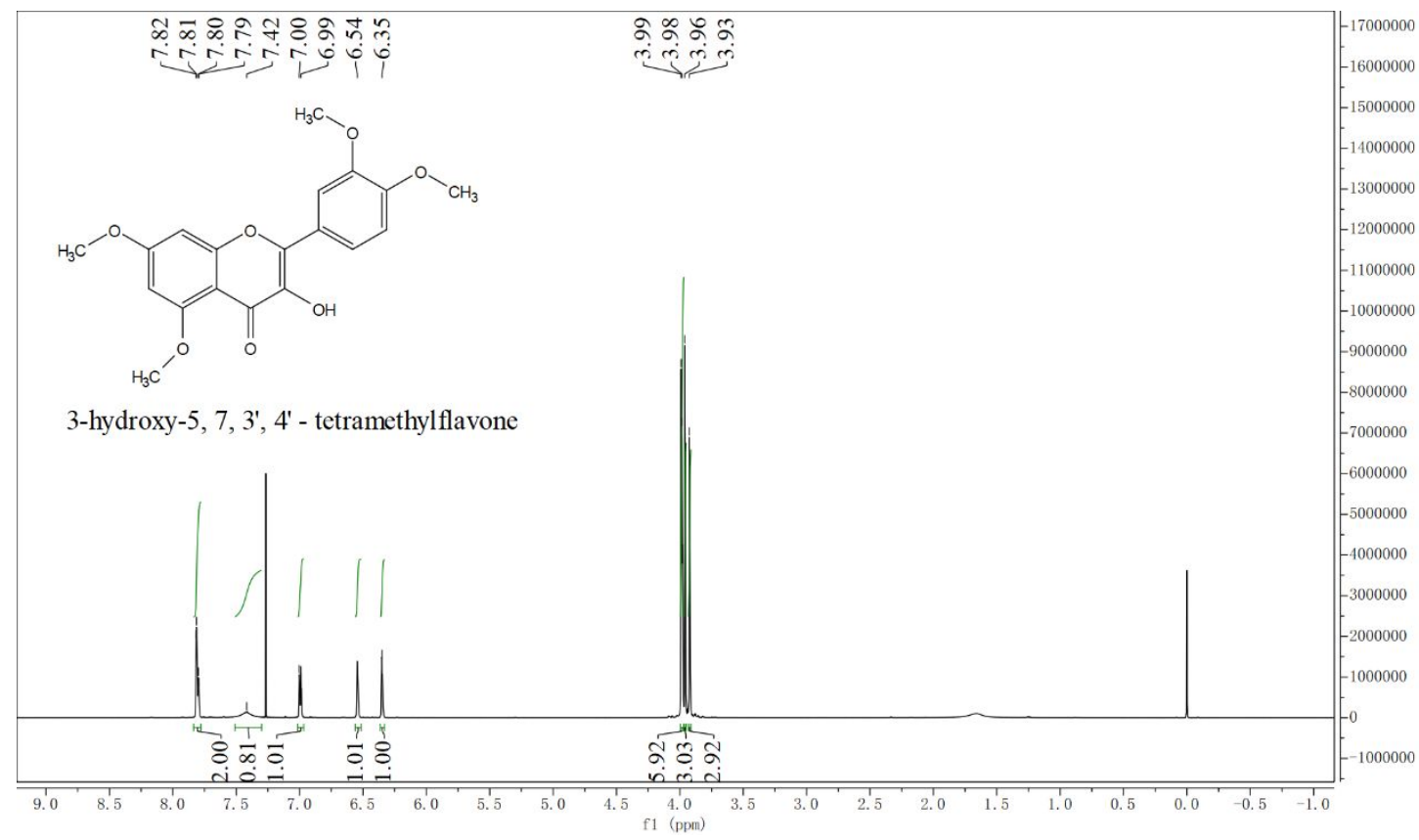

3,5,7,3',4'-Pentamethoxyflavone (9)

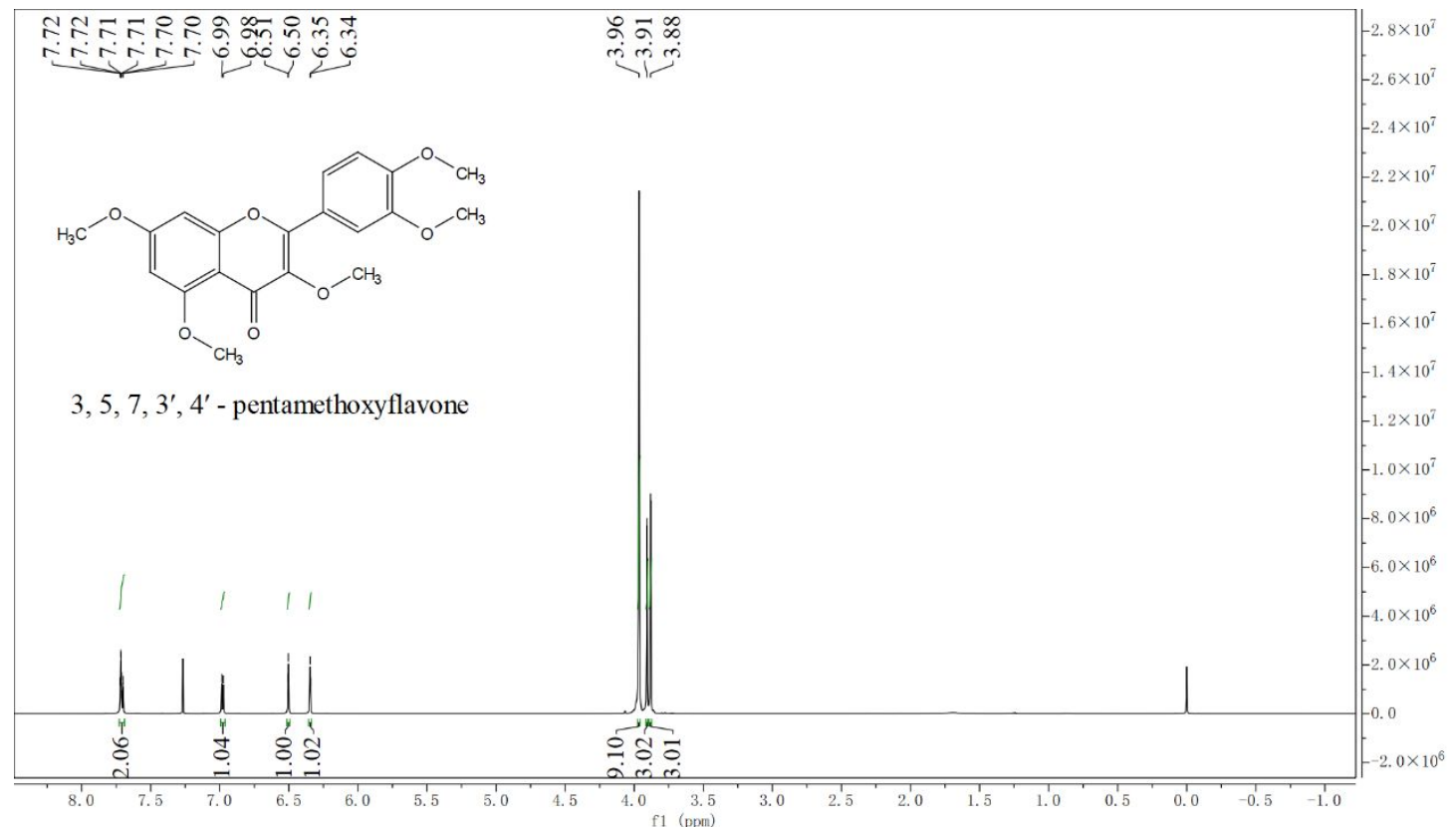

5-Hydroxy-3,7,3',4',5'-Pentamethoxyflavone (10) 


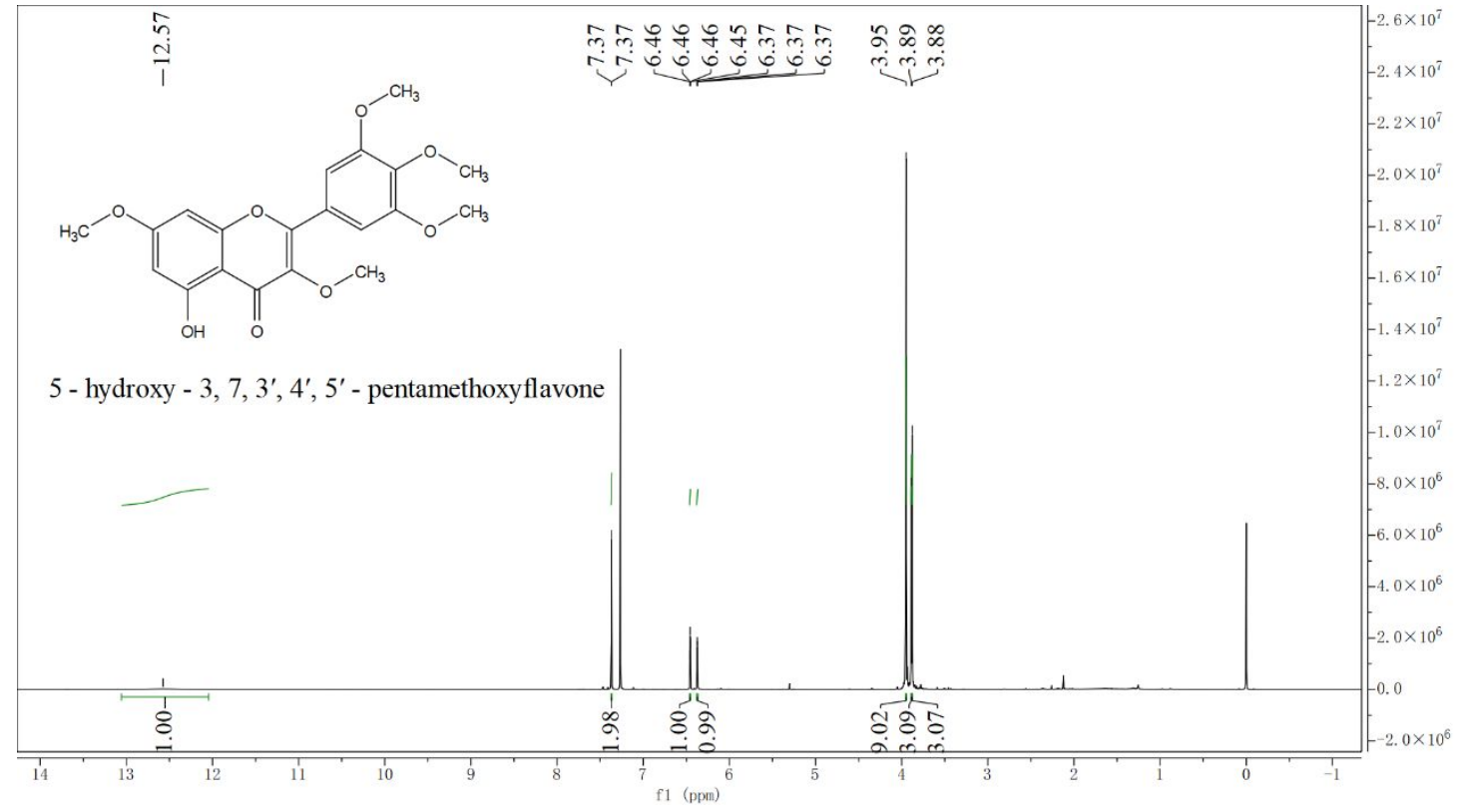

3- Hydroxy-5, 7,3',4',5'-pentamethoxyflavone (11)

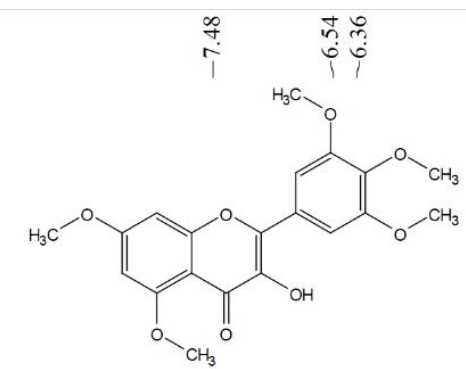

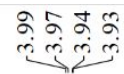

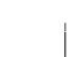

3 - hydroxy - 5, 7, 3', 4', 5' - pentamethoxyflavone

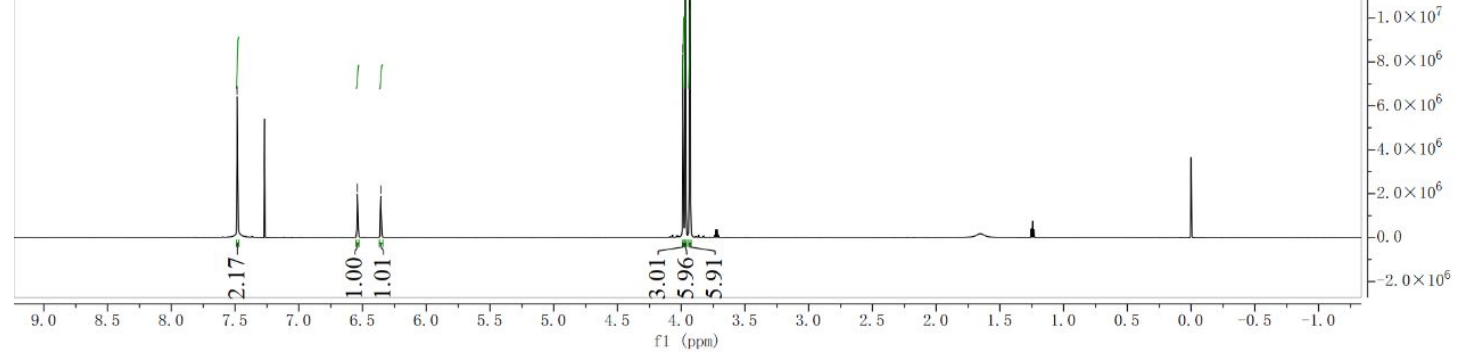

3,5,7,3', $4^{\prime}, 5^{\prime}$-Hexamethoxylflavone (12) 


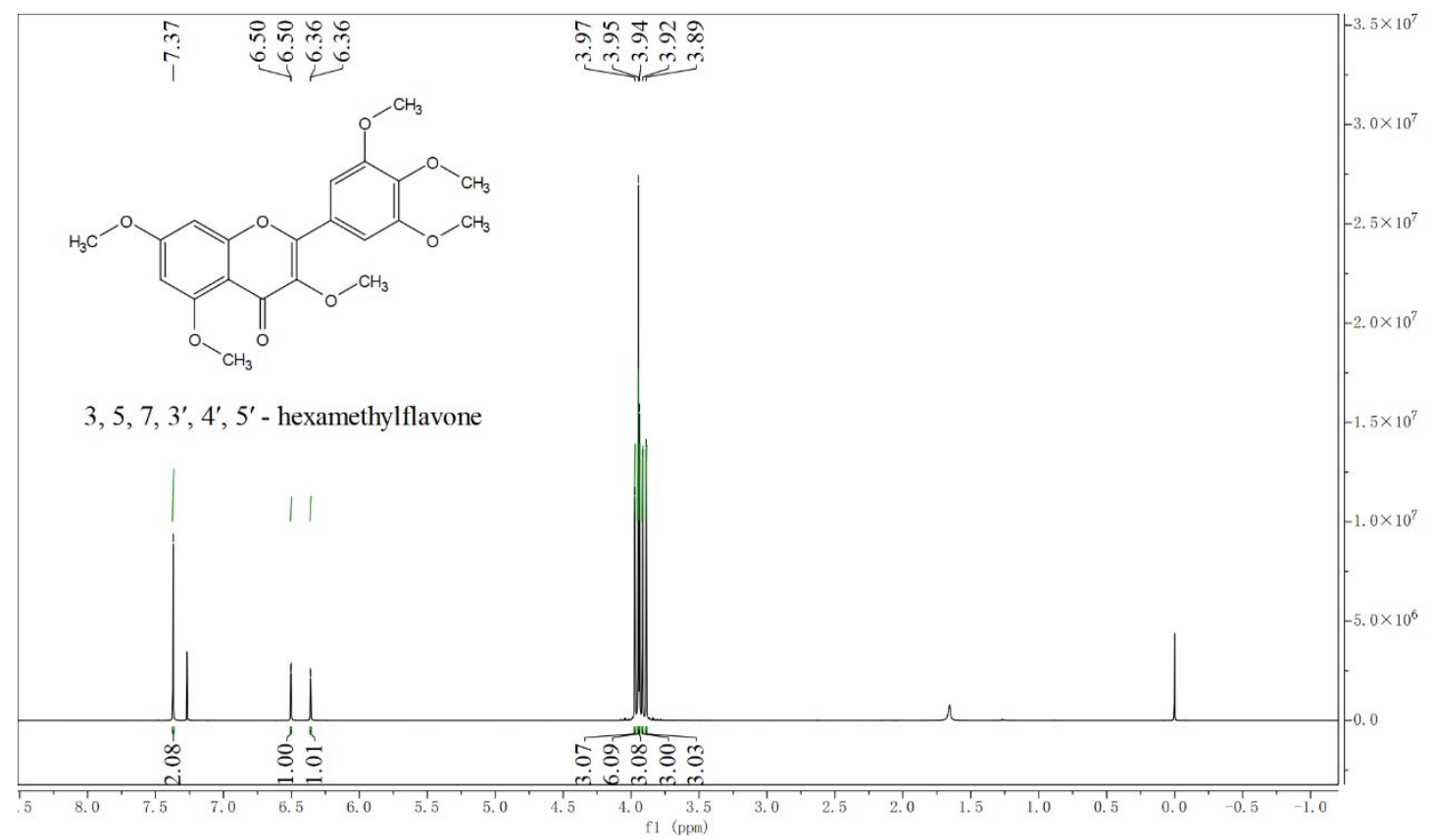

Figure S1. ${ }^{1} \mathrm{H}-\mathrm{NMR}$ of PMFs 1-12

3,5,7,4'-Tetramethoxyflavone (1) $\lambda=326 \mathrm{~nm}$
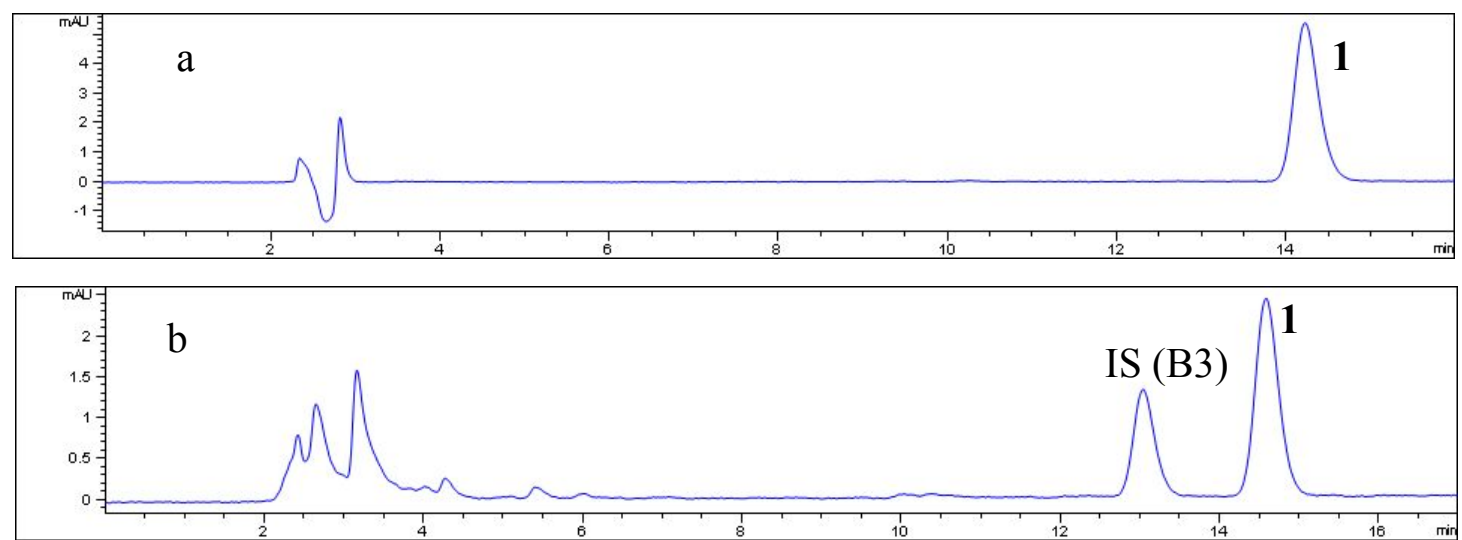

$5,6,7,4^{\prime}$-Tetramethylflavone (2) $\quad \lambda=326 \mathrm{~nm}$ 

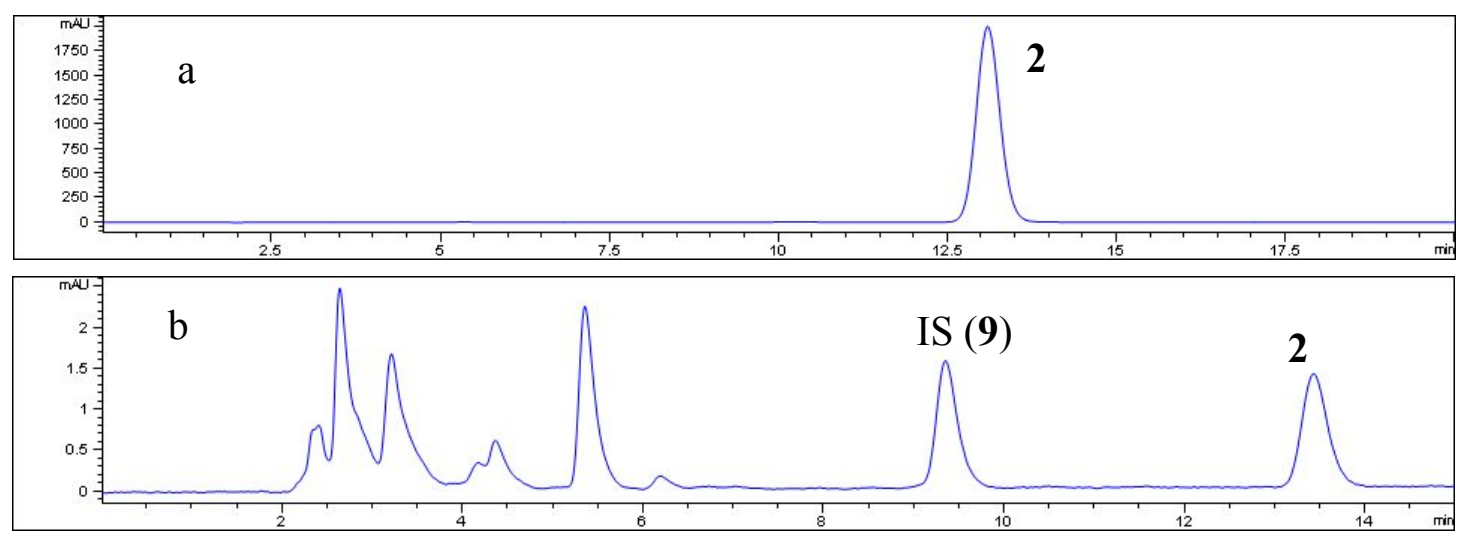

$3,7,3^{\prime}, 4^{\prime}$-Tetramethoxyflavone (3) $\quad \lambda=326 \mathrm{~nm}$
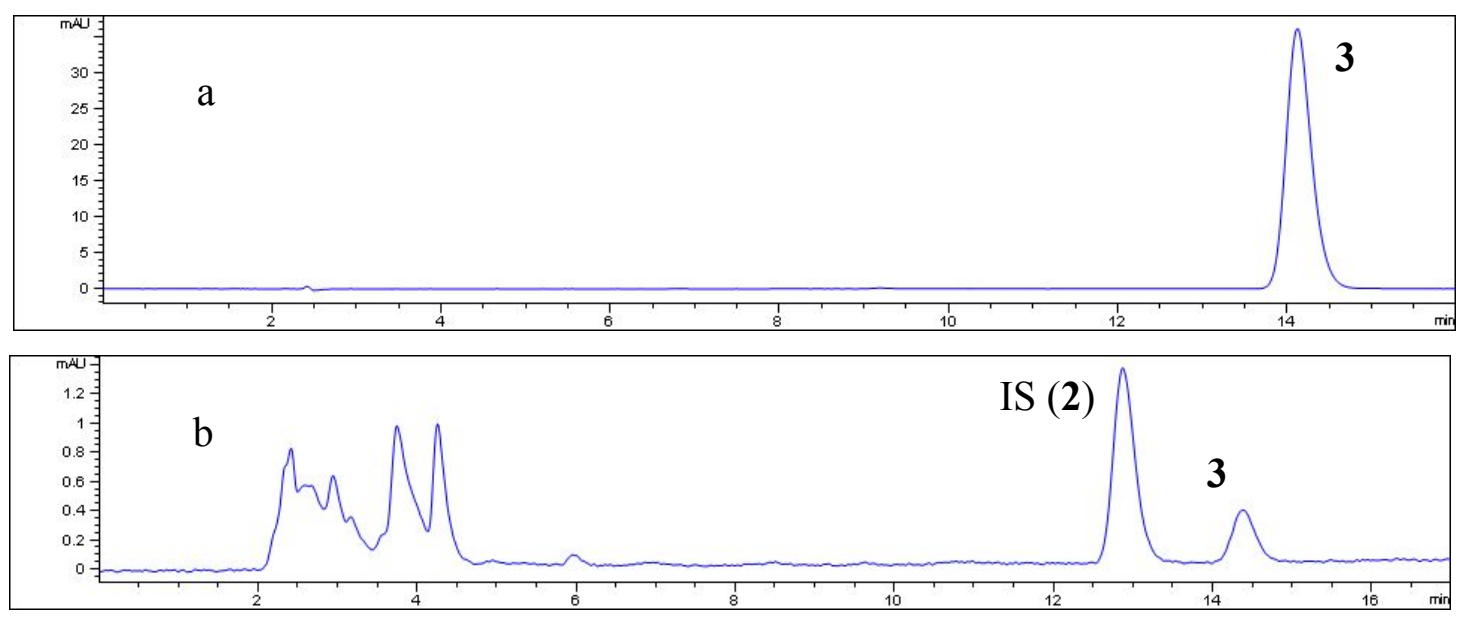

$5,7,3^{\prime}, 4^{\prime}$-Tetramethoxyflavone (4) $\quad \lambda=326 \mathrm{~nm}$
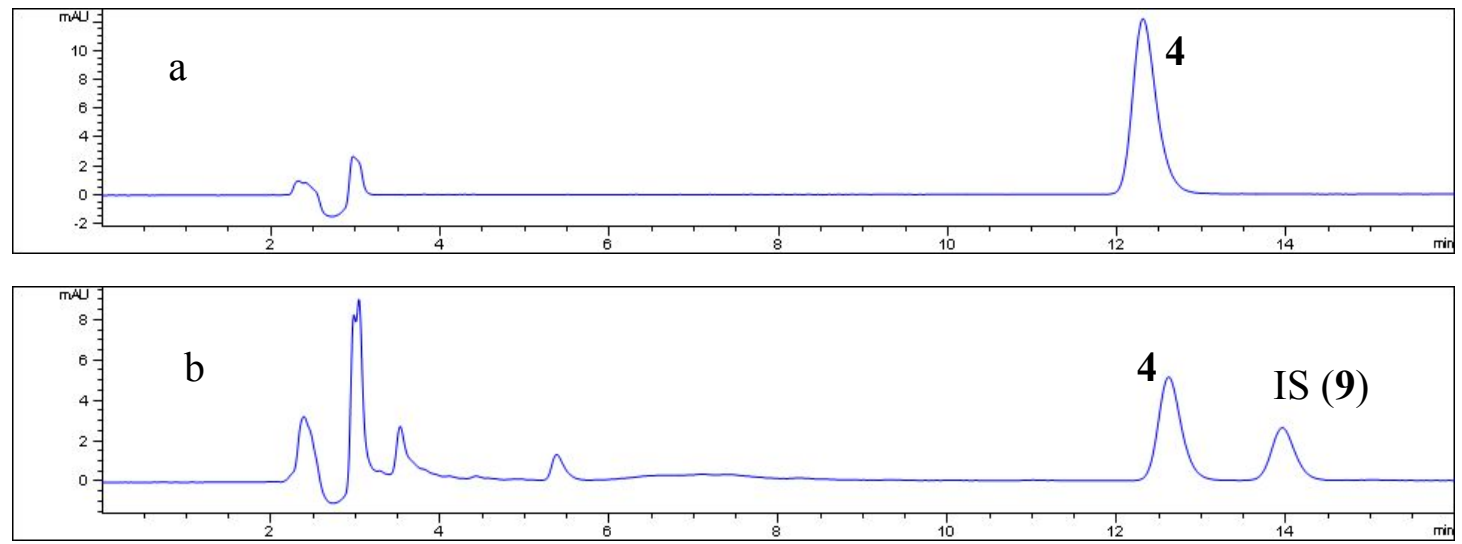

5-Hydroxy-3,7,2',4'-Tetramethoxyflavone (5) $\quad \lambda=326 \mathrm{~nm}$ 

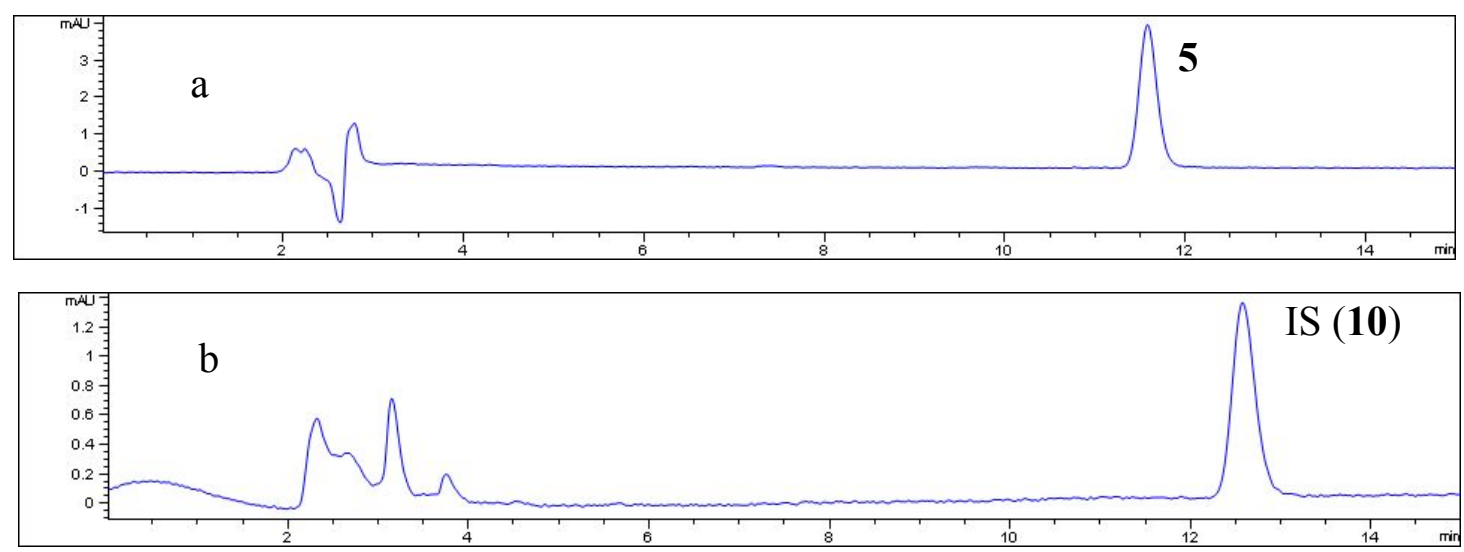

$3,5,7,2^{\prime}, 4^{\prime}$-Pentamethoxyflavone (6) $\lambda=326 \mathrm{~nm}$
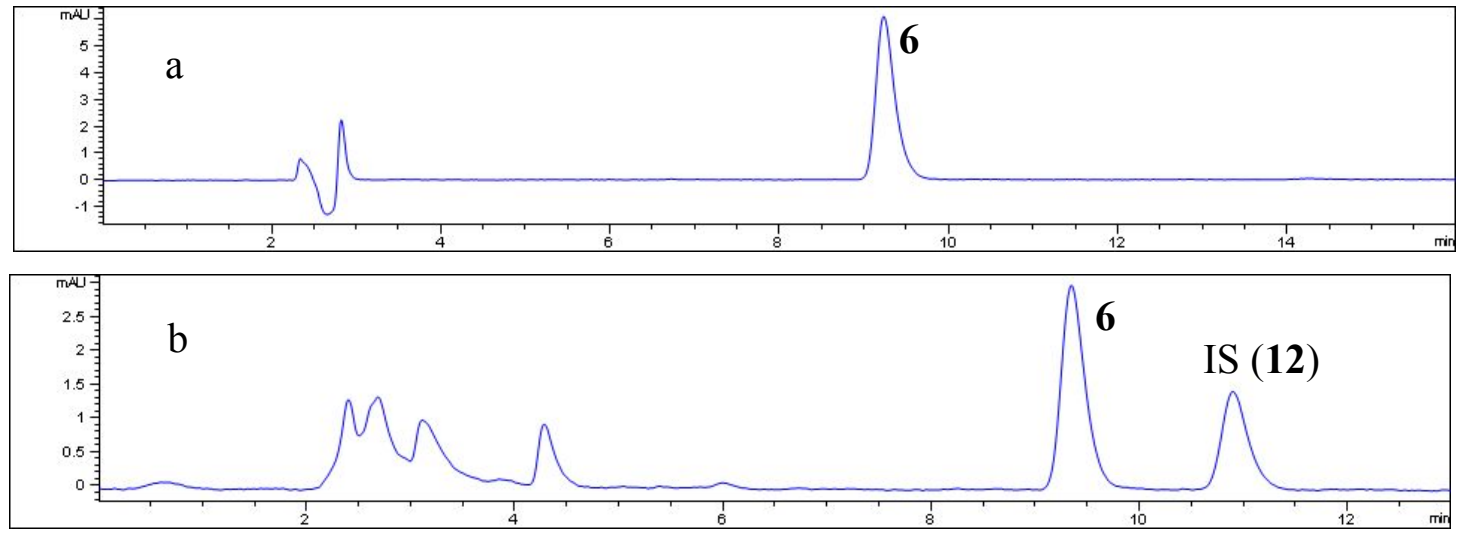

5- Hydroxy-3,7,3',4'-Tetramethoxyflavone (7) $\quad \lambda=326 \mathrm{~nm}$
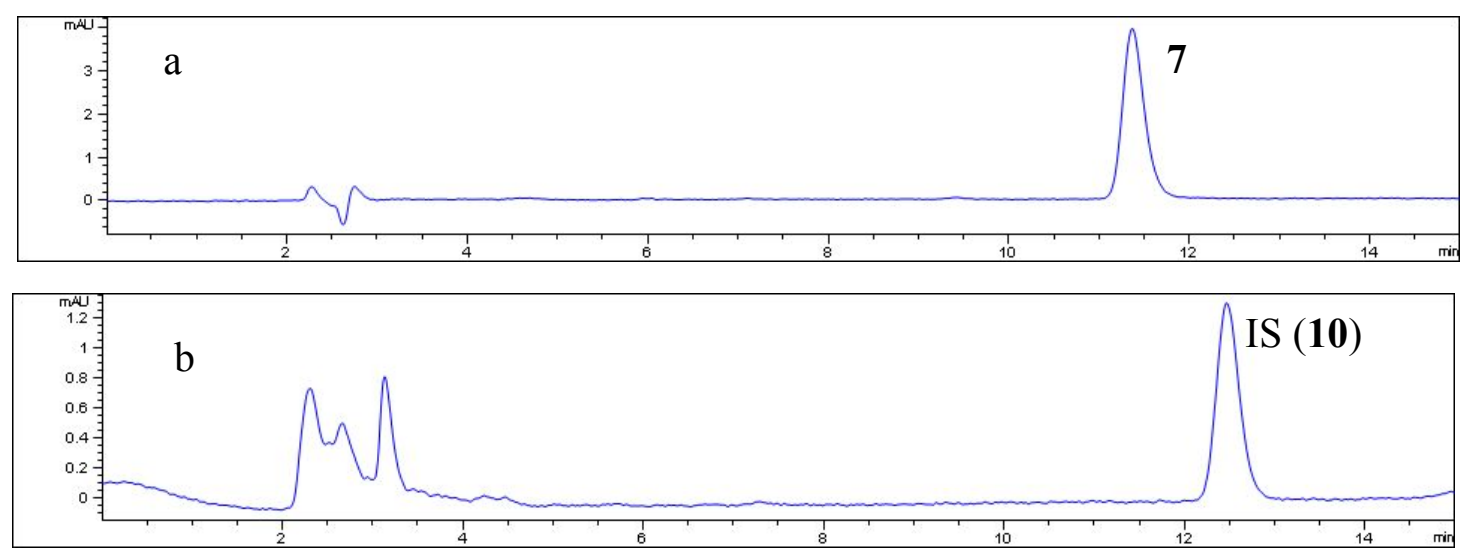

3-Hydroxy-5,7,3',4'-Tetramethylflavone (8) $\lambda=365 \mathrm{~nm}$ 

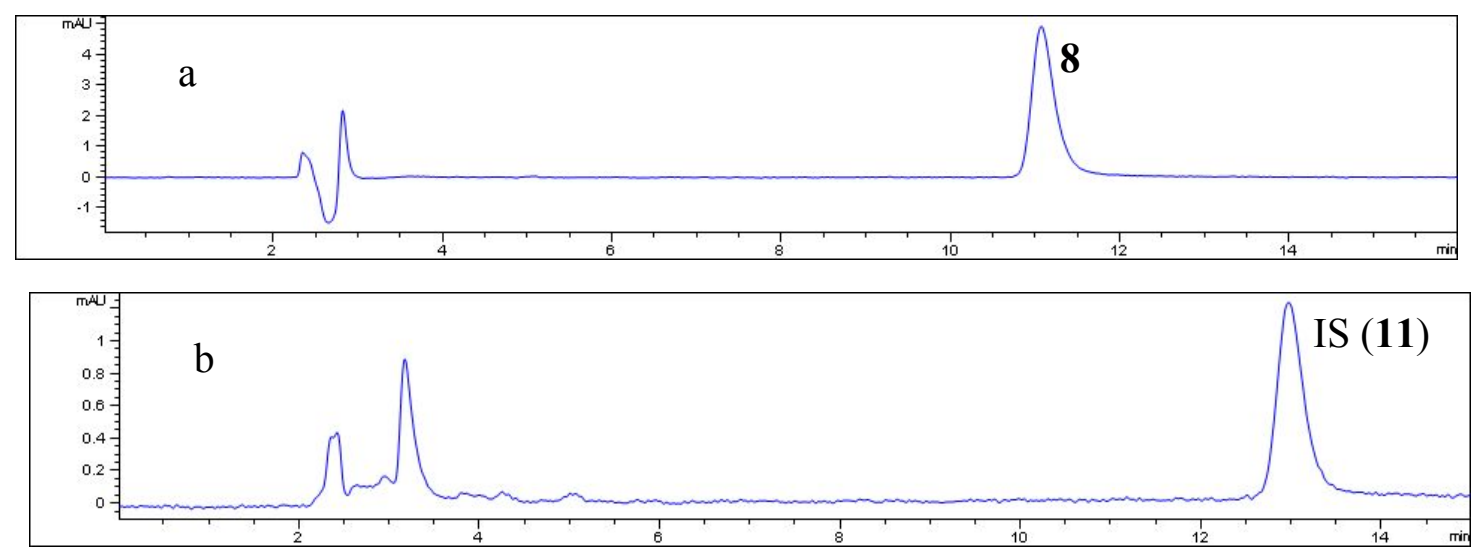

$3,5,7,3^{\prime}, 4^{\prime}$-Pentamethoxyflavone (9) $\quad \lambda=326 \mathrm{~nm}$
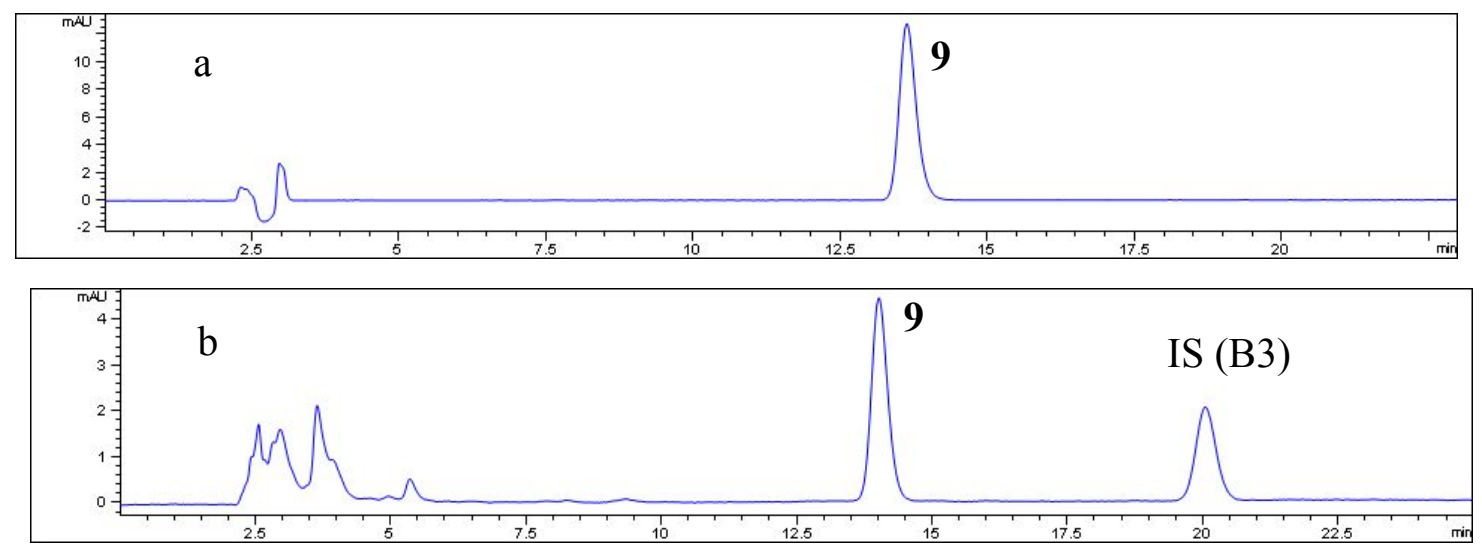

5-Hydroxy-3,7,3',4',5'-Pentamethoxyflavone (10) $\lambda=365 \mathrm{~nm}$
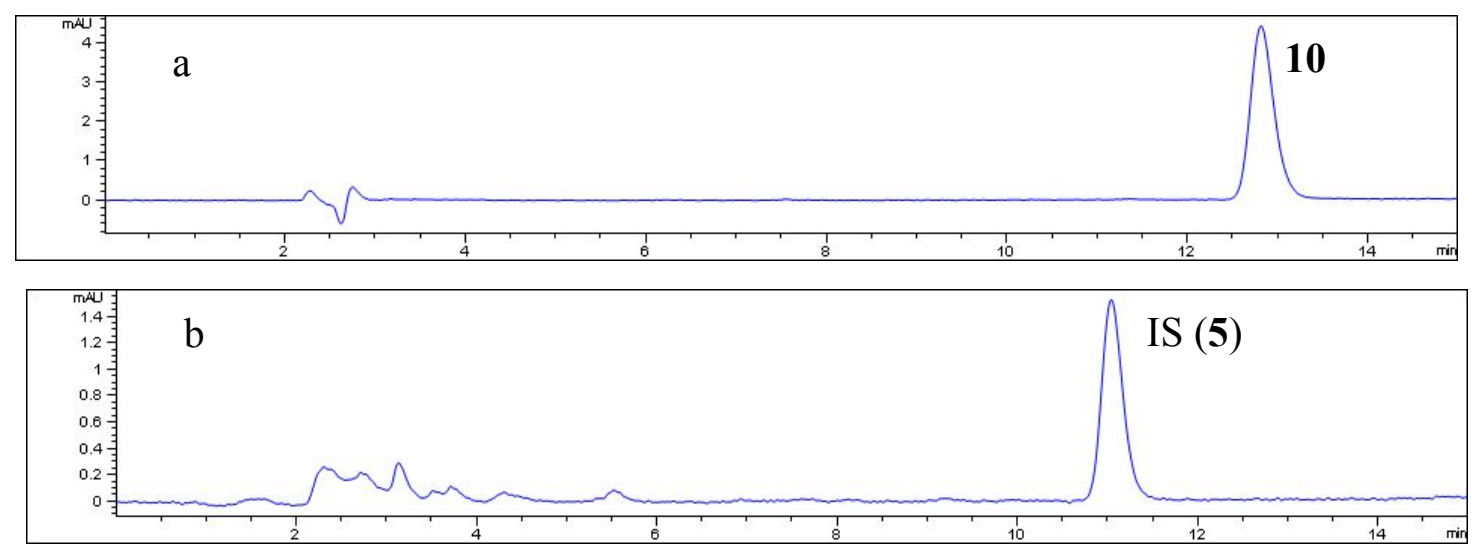

3- Hydroxy-5,7,3',4',5'-pentamethoxyflavone (11) $\lambda=365 \mathrm{~nm}$ 

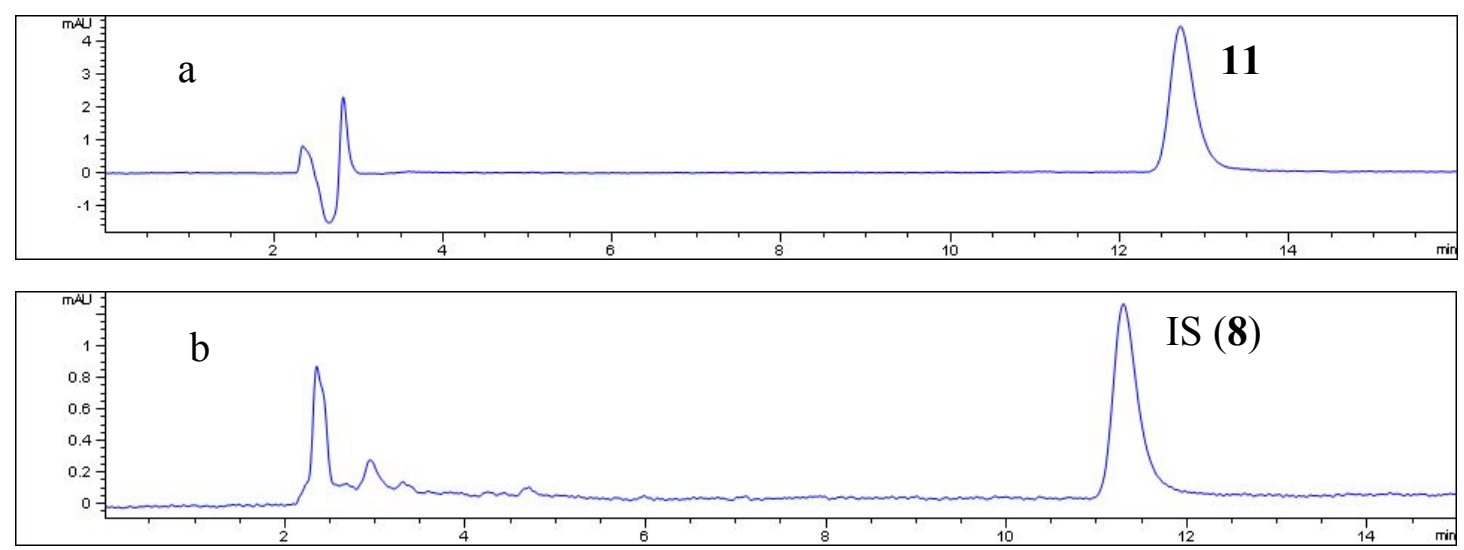

$3,5,7,3^{\prime}, 4^{\prime}, 5^{\prime}-$ Hexamethoxylflavone (12) $\quad \lambda=326 \mathrm{~nm}$
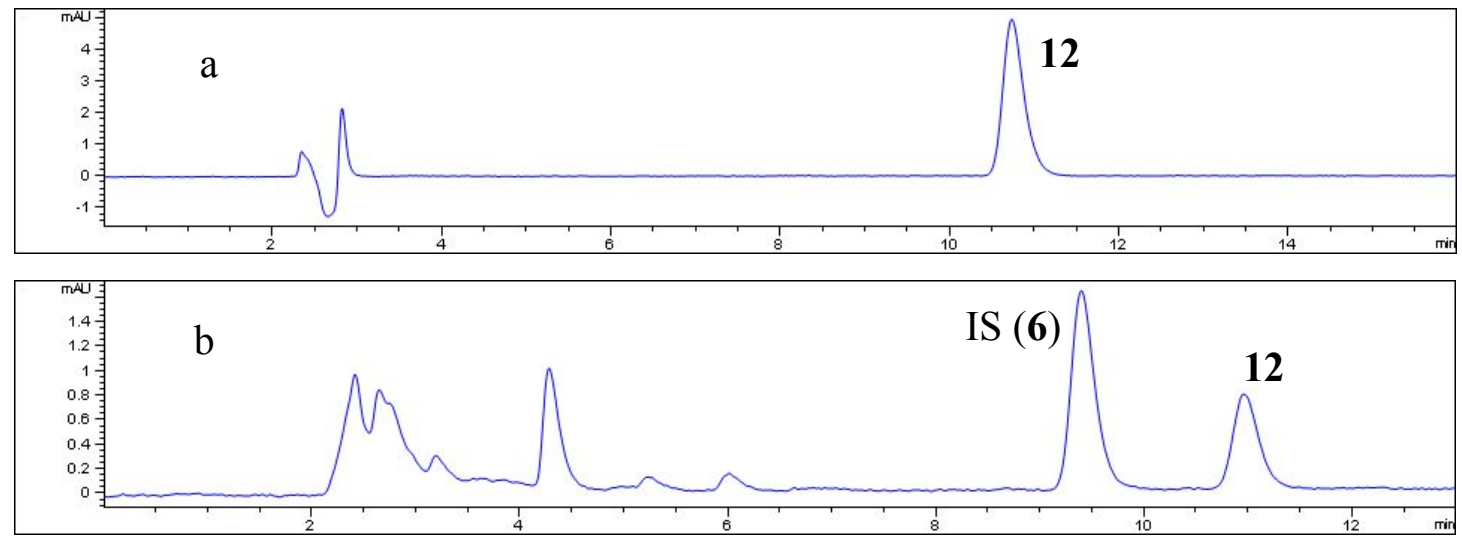

Figure S2. Representative chromatograms of PMFs 1-12 in pharmacokinetics experiment. Note: a indicates standard substance; $\mathrm{b}$ indicates blood sample 3,5,7,4'-Tetramethoxyflavone (1)

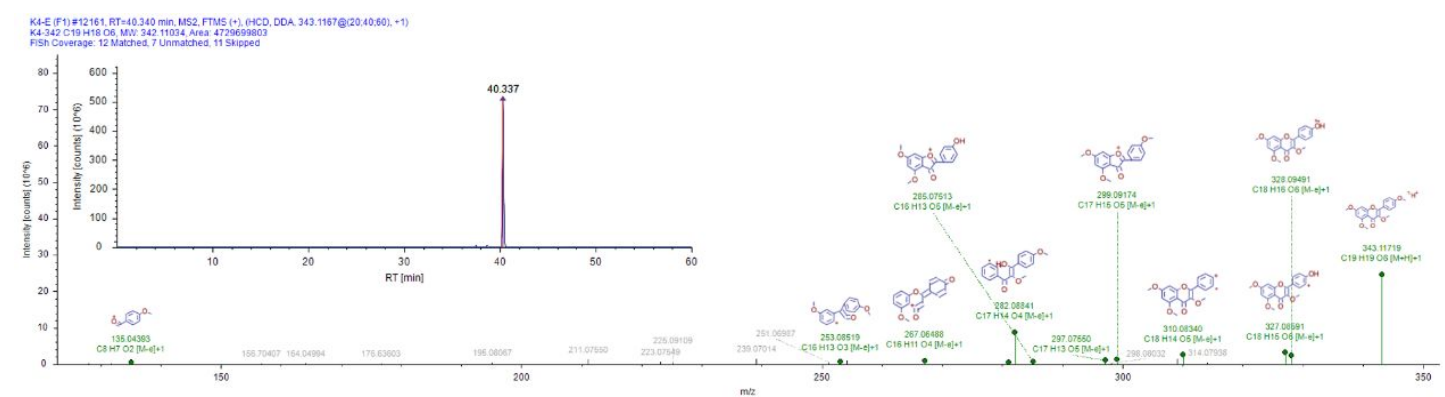

5,6,7,4'-Tetramethylflavone (2) 


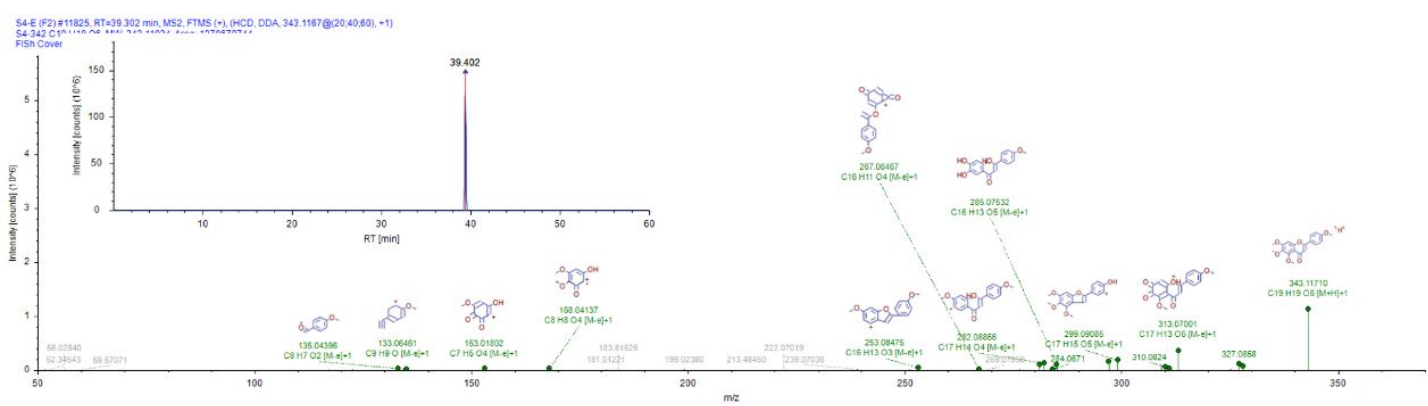

3,7,3',4'-Tetramethoxyflavone (3)

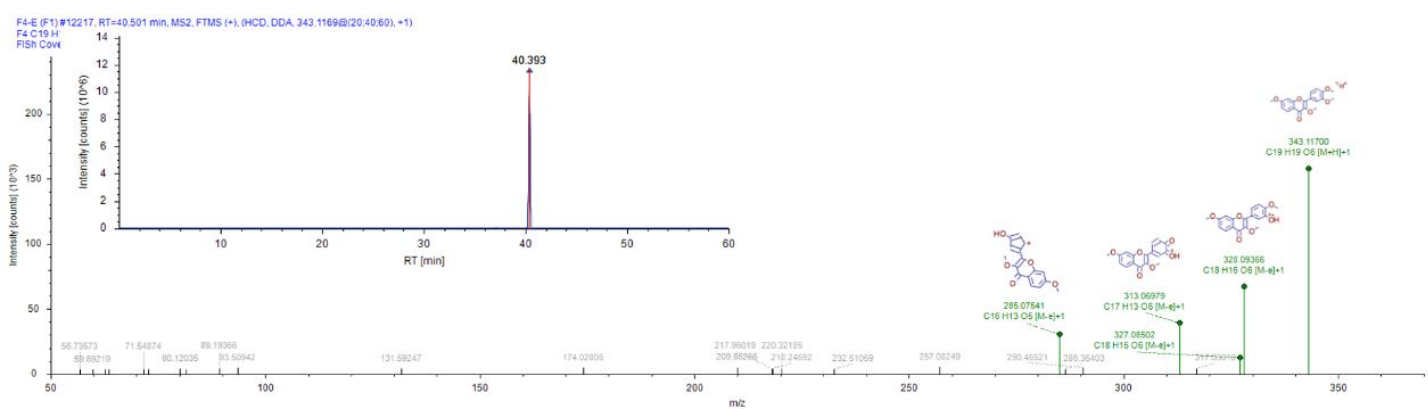

5,7,3', $4^{\prime}$-Tetramethoxyflavone (4)

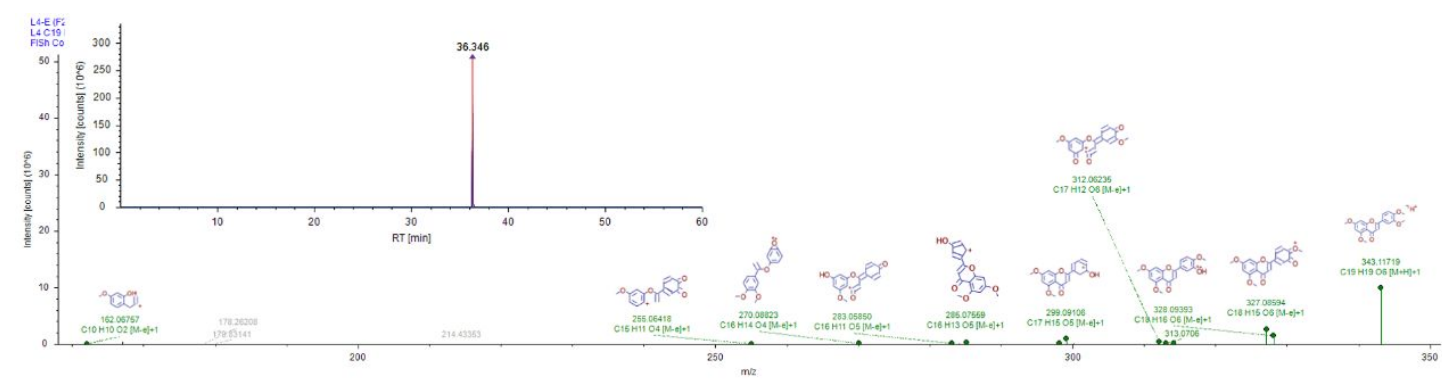

5-Hydroxy-3,7,2',4'-Tetramethoxyflavone (5) 


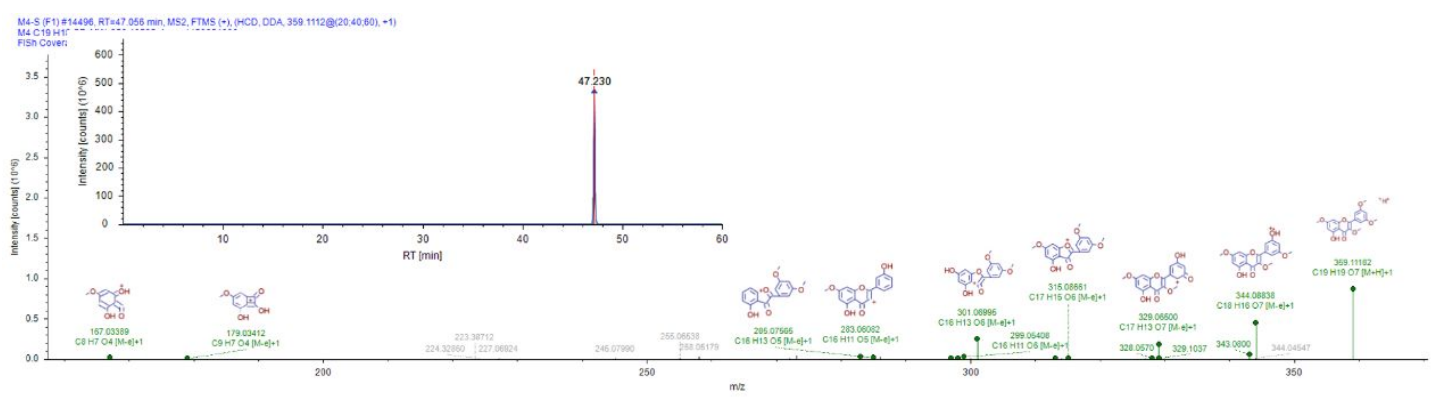

3,5,7,2',4'-Pentamethoxyflavone (6)

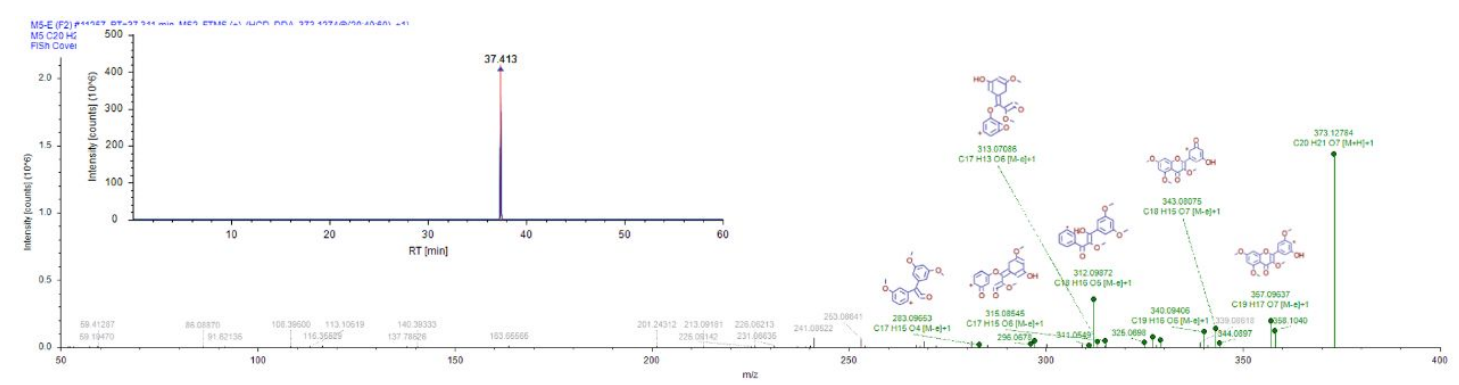

5- Hydroxy-3, 7,3',4'-Tetramethoxyflavone (7)

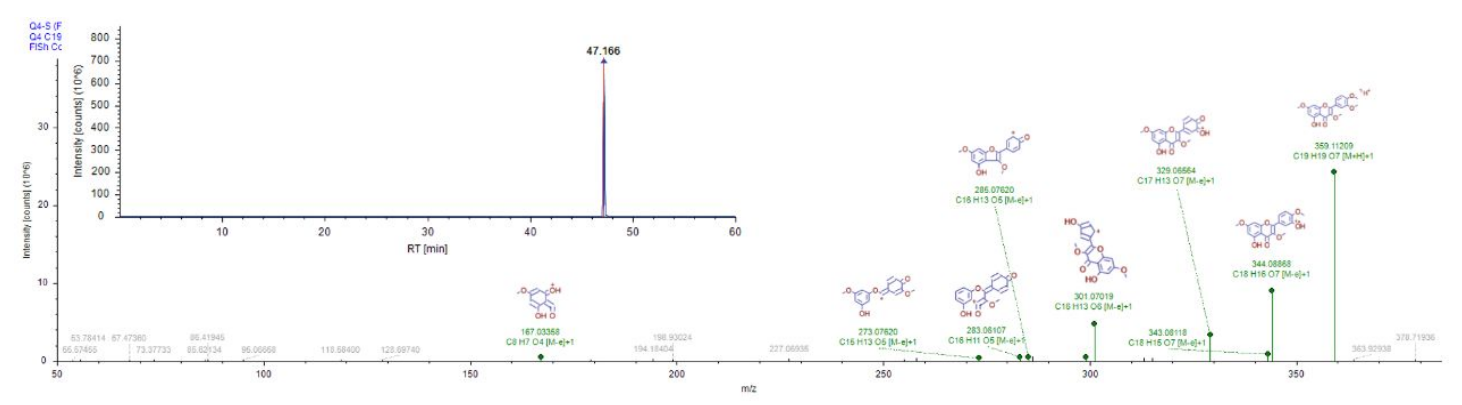

3-Hydroxy-5, 7,3',4'-Tetramethylflavone (8)

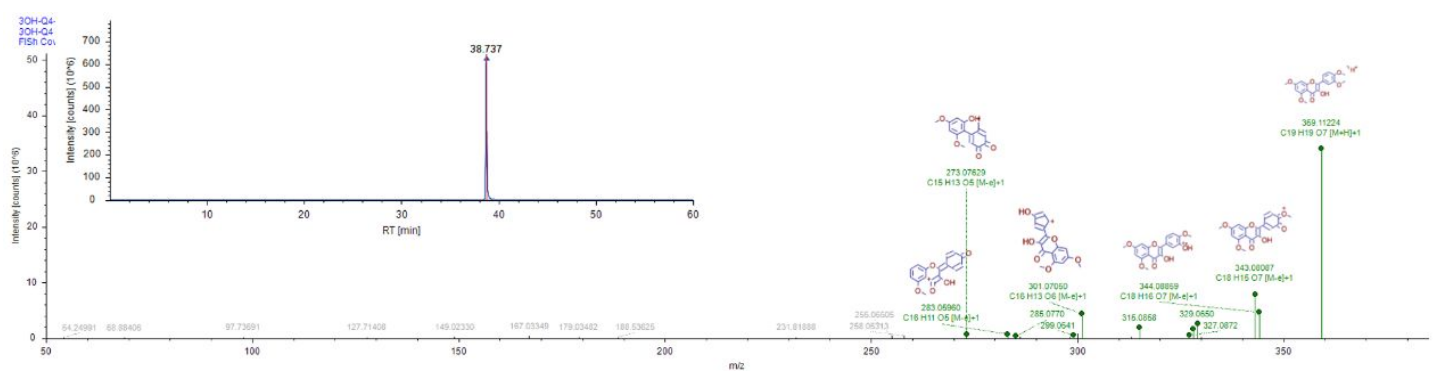

3,5,7,3',4'-Pentamethoxyflavone (9) 


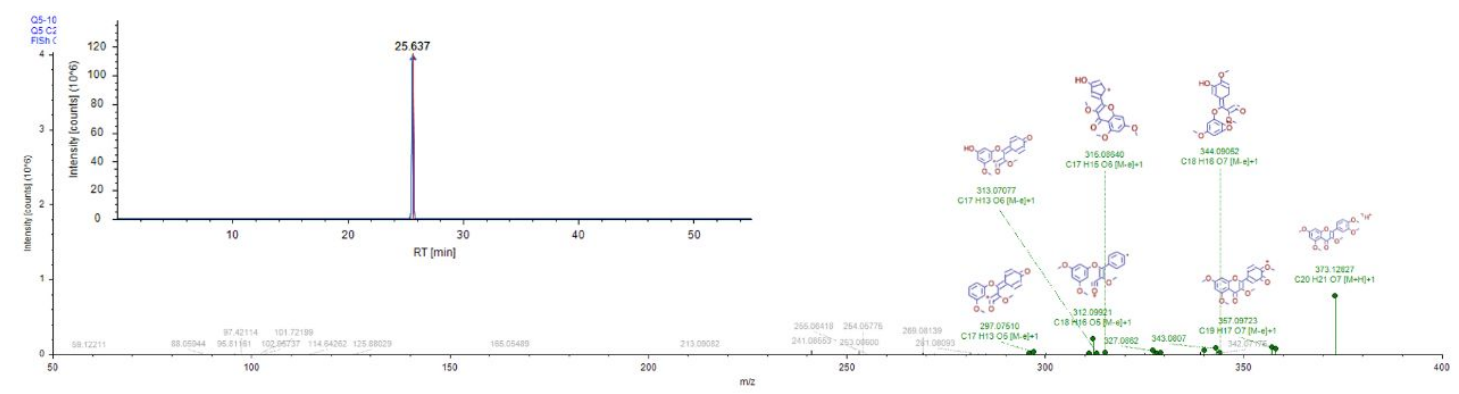

5-Hydroxy-3, 7,3',4',5'-Pentamethoxyflavone (10)

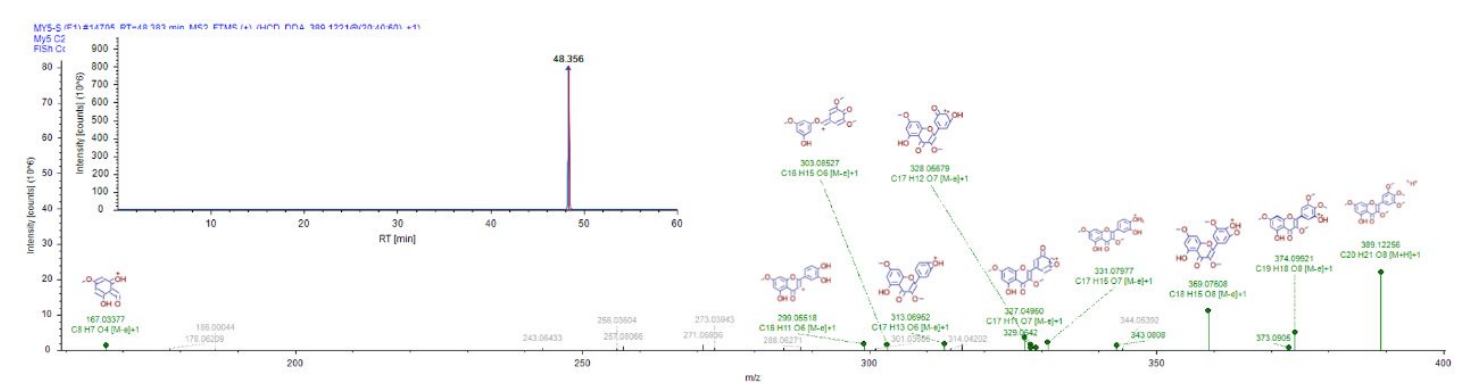

3- Hydroxy-5,7,3',4',5'-pentamethoxyflavone (11)

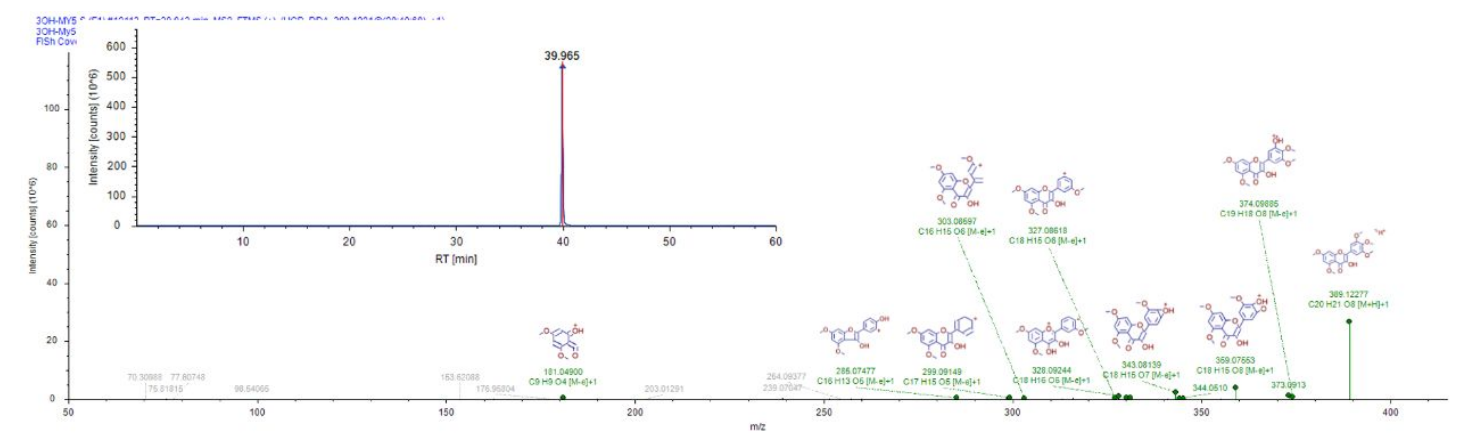

3,5,7,3', $4^{\prime}, 5^{\prime}$-Hexamethoxylflavone (12)

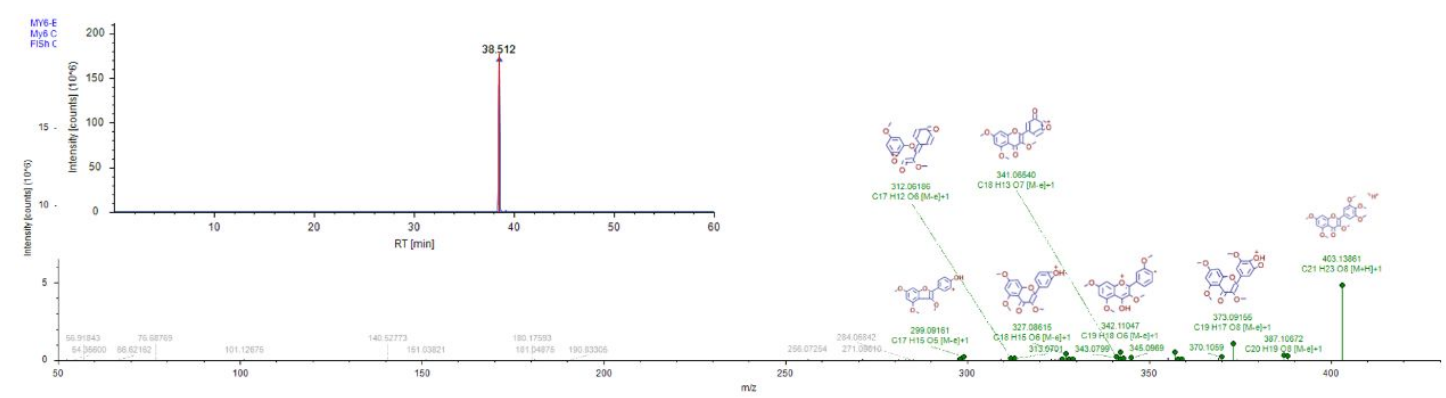

Figure S3. UPLC-Q Exactive Orbitrap HRMS of PMFs 1-12 
Table S1. Review of the biological activities of PMFs 1-12 


\begin{tabular}{|c|c|}
\hline PMF & Biological activity \\
\hline 1 & Inhibits epoxide hydrolase ${ }^{1}$, anticancer ${ }^{2}$, Anti-inflammation ${ }^{3,4}$, Anti-mutagenic $^{5}$ \\
\hline 2 & Anticancer ${ }^{6,7}$, Anti-inflammation ${ }^{8,9}$ \\
\hline 3 & Anticancer ${ }^{10}$, Anti-inflammation ${ }^{11}, \alpha$-glucosidase inhibitory activity ${ }^{12}$ \\
\hline 4 & $\begin{array}{l}\text { Anti-inflammation }{ }^{13,14}, \alpha \text {-glucosidase inhibitory activity }{ }^{15} \text {, chondroprotective activity }{ }^{16} \text {, } \\
\text { anti-angiogenesis }{ }^{17}\end{array}$ \\
\hline 5 & Anticancer ${ }^{18}$ \\
\hline 6 & Anticancer ${ }^{19,20}$, inhibit $\mathrm{A} 3$ receptor $^{21}$ \\
\hline 7 & Anti-inflammation ${ }^{22}$, Anticancer ${ }^{23-25}$ \\
\hline 8 & Antiviral $^{26}$, Anticancer ${ }^{27}$ \\
\hline 9 & $\begin{array}{l}\text { activation of Sestrin } 2^{28} \text {, Anti-inflammation }{ }^{29} \text {, anti-obesity }{ }^{30} \text {, Inhibits } \beta \text {-secretase }{ }^{31} \text {, } \\
\text { antioxidative damage }{ }^{32} \text { ameliorate metabolic disorders }{ }^{33}\end{array}$ \\
\hline 10 & Anticancer $^{34}$ \\
\hline 11 & Anticancer ${ }^{35}$, Anti-inflammation ${ }^{36}$ \\
\hline 12 & Inhibits P-glycoprotein ${ }^{37}$, Antidiabetogenic ${ }^{38}$, Antioxidant and anti-radical ${ }^{39}$ \\
\hline
\end{tabular}


Table S2. Systematic review on natural PMFs and their sources

\begin{tabular}{|c|c|c|c|}
\hline Compound & $\begin{array}{l}\text { Source } \\
\text { (genus)/References }\end{array}$ & Compound & $\begin{array}{l}\text { Source } \\
\text { (genus)/References }\end{array}$ \\
\hline $5,6,7,8$-tetramethoxyflavone & Scutellaria ${ }^{40}$ & $\begin{array}{l}\text { 3, 5, 7, 4'-tetramethoxyflavone } \\
\text { (PMF 1) }\end{array}$ & $\begin{array}{l}\text { Citrus }^{41}, \\
\text { Kaempferia }^{43-45}, \\
\text { Micromelum }^{46}, \text { cissus }^{47} \text {, } \\
\text { Calophyllum }^{48}, \\
\text { Manglietia }^{49}, \\
\text { Hedychium }^{50}\end{array}$ \\
\hline $3,6,7,4$ '-tetramethoxyflavone & Citrus $^{51,52}$ & $\begin{array}{l}\text { 3, 7, 3', 4'-tetramethoxyflavone } \\
\text { (PMF 3) }\end{array}$ & $\begin{array}{l}\text { Commelina }^{53} \text {, } \\
\text { Pongamia }^{54,55} \text { Derris }^{56,57} \text {, } \\
\text { Ligularia }^{58}\end{array}$ \\
\hline $5,6,2^{\prime}, 3^{\prime}$-tetramethoxyflavone & Casimiroa $^{59}$ & $\begin{array}{l}5,6,2^{\prime}, 6^{\prime} \text {-tetramethoxyflavone } \\
\text { (zapotin) }\end{array}$ & $\begin{array}{l}\text { Casimiroa }^{60-62} \\
\text { Struthiola }^{63}\end{array}$ \\
\hline $5,6,3^{\prime}, 4^{\prime}$-tetramethoxyflavone & Casimiroa $^{64}$, Ardisia $^{65}$ & $\begin{array}{l}5,6,3^{\prime}, 5^{\prime} \text {-tetramethoxyflavone } \\
\text { (cerrosillin) }\end{array}$ & Casimiroa $^{64}$ \\
\hline $5,6,7, \quad 4^{\prime}$-tetramethoxyflavone & Citrus $^{66,67}$, Orthosiphon ${ }^{68}$, & $5,7,8,4^{\prime}$-tetramethoxyflavone & Citrus $^{70-72}$ \\
\hline
\end{tabular}




(PMF 2) Chromolaena ${ }^{69}$

$5,7,8,2^{\prime}$-tetramethoxyflavone Andrographis ${ }^{73}$

$5,7,2^{\prime}, 3^{\prime}$-tetramethoxyflavone $\quad$ Andrographis $^{74}$

\begin{tabular}{|c|c|c|c|}
\hline \multirow{3}{*}{$5,7,2^{\prime}, 4^{\prime}$-tetramethoxyflavone } & \multirow{3}{*}{ Terminalia $^{75}$} & & Citrus $^{41,76}$, Bauhinia ${ }^{77,78}$, \\
\hline & & $\begin{array}{l}5,7,3^{\prime}, 4^{\prime} \text {-tetramethoxyflavone } \\
\text { (PMF 4) }\end{array}$ & 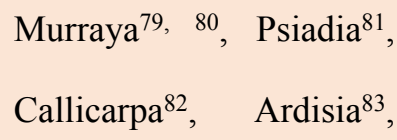 \\
\hline & & & Kaempferia $^{84}$, Ocimum $^{85}$ \\
\hline $5,7,3^{\prime}, 5^{\prime}$-tetramethoxyflavone & Casimiroa $^{62}$ & $5,2^{\prime}, 3^{\prime}, 4^{\prime}$-tetramethoxyflavone & Casimiroa $^{86}$ \\
\hline $6,7,8,4$ '-tetramethoxyflavone & Citrus $^{87}$ & $7,8,2^{\prime}, 5^{\prime}$-tetramethoxyflavone & Andrographis ${ }^{73}$ \\
\hline $7,2^{\prime}, 4^{\prime}, 5^{\prime}$-tetramethoxyflavone & Calliandra $^{88}$ & $\begin{array}{l}\text { 3-hydroxy-5, 6, 7, 4'- } \\
\text { tetramethoxyflavone }\end{array}$ & Citrus $^{51}$ \\
\hline
\end{tabular}

3-hydroxy-5, 7, 3', 4'-

tetramethoxyflavone (PMF 8)

Citrus $^{89}$, Laggera $^{90}$

5-hydroxy-3, 7, 3', 4'-

tetramethoxyflavone (PMF 7)

Citrus $^{91}$, Kaempferia ${ }^{92}$

5-hydroxy-3, 7, 8, 4'-

5-hydroxy-3, 7, 2', 4'-

tetramethoxyflavone (PMF 5)

$\begin{array}{lll}\text { Mortonia }^{93} & \text { tetramethoxyflavone } & \text { Micromelum }\end{array}$

(flindulatin)

5-hydroxy-7, 8, 3', 4'- $\quad$ Citrus $^{89,}{ }^{85}$, Peperomia $^{96}$

5-hydroxy-6, 7, 3', 4'-

tetramethoxyflavone

Citrus $^{97,}$ 98, Murraya $^{79}$,

tetramethoxyflavone

Citrus $^{89,95}$, Peperomia ${ }^{96}$

(flindulatin)

Micromelum

\begin{tabular}{|c|c|c|c|}
\hline $\begin{array}{l}\text { 5-hydroxy-3, 6, 7, 4'- } \\
\text { tetramethoxyflavone }\end{array}$ & $\begin{array}{l}\text { Gardenia }^{103}, \text { Teucrium }^{104} \text {, } \\
\text { Callicarpa }^{105} \text {, } \\
\text { Micromelum }^{106}\end{array}$ & $\begin{array}{l}\text { 5-hydroxy-3, 6, 7, 8- } \\
\text { tetramethoxyflavone }\end{array}$ & Citrus $^{98}$ \\
\hline $\begin{array}{l}\text { 5-hydroxy-7, 2', 4', 5'- } \\
\text { tetramethoxyflavone }\end{array}$ & Calliandra $^{88}$ & $\begin{array}{l}\text { 5-hydroxy-7, 3', 4', 5'- } \\
\text { tetramethoxyflavone }\end{array}$ & Murraya $^{79}$ \\
\hline $\begin{array}{l}\text { 5-hydroxy-7, 8, 2', 3'- } \\
\text { tetramethoxyflavone }\end{array}$ & Andrographis ${ }^{73,74}$ & $\begin{array}{l}\text { 5-hydroxy-7, 8, 2', 4'- } \\
\text { tetramethoxyflavone }\end{array}$ & Limnophila ${ }^{107}$ \\
\hline $\begin{array}{l}\text { 5-hydroxy-7, 8, 2', 5'- } \\
\text { tetramethoxyflavone }\end{array}$ & Andrographis ${ }^{108}$ & $\begin{array}{l}\text { 5-hydroxy-7, 8, 2', 6'- } \\
\text { tetramethoxyflavone }\end{array}$ & Andrographis ${ }^{73}$ \\
\hline 5-hydroxy- $6,7,8,4^{\prime}-$ & Citrus $^{109}$, Ocimum $^{85}$, & 6-hydroxy-5, 7, 8, 4'- & Citrus $^{89,98}$ \\
\hline
\end{tabular}




\begin{tabular}{|c|c|c|c|}
\hline tetramethoxyflavone (gardenin B) & Gardenia $^{110,111}$ & tetramethoxyflavone & \\
\hline $\begin{array}{l}\text { 6-hydroxy-3, 5, 7, 4'- } \\
\text { tetramethoxyflavone }\end{array}$ & Blumea $^{112}$ & $\begin{array}{l}\text { 7-hydroxy-3, 6, 3', 4'- } \\
\text { tetramethoxyflavone }\end{array}$ & Ajuga ${ }^{113}$ \\
\hline $\begin{array}{l}\text { 7-hydroxy-5, 6, 8, 4'- } \\
\text { tetramethoxyflavone }\end{array}$ & Citrus $^{89}$ & $\begin{array}{l}\text { 7-hydroxy-3, 5, 3', 4'- } \\
\text { tetramethoxyflavone }\end{array}$ & Distemonanthus ${ }^{114}$ \\
\hline $\begin{array}{l}\text { 7-hydroxy-5, 6, 3', 4'- } \\
\text { tetramethoxyflavone }\end{array}$ & Citrus $^{115}$ & $\begin{array}{l}\text { 7-hydroxy-6, 8, 3', 4'- } \\
\text { tetramethoxyflavone }\end{array}$ & Euryops $^{116}$ \\
\hline $\begin{array}{l}\text { 8-hydroxy-7, 3', 4', 5'- } \\
\text { tetramethoxyflavone }\end{array}$ & Muntingia ${ }^{117}$ & $\begin{array}{l}\text { 3'-hydroxy-5, 6, 7, 4'- } \\
\text { tetramethoxyflavone }\end{array}$ & orthosiphon $^{118}$ \\
\hline $\begin{array}{l}\text { 3'-hydroxy-3, 7, 8, 4'- } \\
\text { tetramethoxyflavone }\end{array}$ & Mimosa $^{119}$ & $\begin{array}{l}\text { 3'-hydroxy-5, 7, 4', 5'- } \\
\text { tetramethoxyflavone }\end{array}$ & Murraya $^{80}$ \\
\hline $\begin{array}{l}\text { 4'-hydroxy-3, 5, 6, 7- } \\
\text { tetramethoxyflavone }\end{array}$ & Varthemia $^{120}$ & $\begin{array}{l}\text { 4'-hydroxy-5, 6, 7, 3'- } \\
\text { tetramethoxyflavone (agecorynin E) }\end{array}$ & Citrus $^{89}$, Ageratum $^{121}$ \\
\hline $\begin{array}{l}\text { 4'-hydroxy-5, 6, 7, 8- } \\
\text { tetramethoxyflavone }\end{array}$ & Citrus $^{122}$ & $\begin{array}{l}\text { 4'-hydroxy-7, 8, 3', 5'- } \\
\text { tetramethoxyflavone }\end{array}$ & Muntingia ${ }^{123}$ \\
\hline $\begin{array}{l}3,5 \text {-dihydroxy- } 6,7,3^{\prime}, 4^{\prime}- \\
\text { tetramethoxyflavone }\end{array}$ & $\begin{array}{l}\text { Pluchea }^{124}, \text { Artemisia }^{125} \text {, } \\
\text { Rumex }^{126} \text { Celastrus }^{127} \text {, } \\
\text { Laggera }^{128}\end{array}$ & $\begin{array}{l}5,6 \text {-dihydroxy-7, 8, 3', 4'- } \\
\text { tetramethoxyflavone }\end{array}$ & Mentha $^{129}$ \\
\hline $\begin{array}{l}\text { 5, 7-dihydroxy-3, 8, 3' } \text { 4' }^{\prime}- \\
\text { tetramethoxyflavone }\end{array}$ & Parastrephia $^{130}$ & $\begin{array}{l}\text { 5, 7-dihydroxy- } 6,8,3^{\prime}, 4^{\prime}- \\
\text { tetramethoxyflavone }\end{array}$ & $\begin{array}{l}\text { Citrus }^{131}, \\
\text { Mentha }^{132}, \text { Helianthus }^{133} \\
\end{array}$ \\
\hline $\begin{array}{l}\text { 5, 7-dihydroxy- } 6,2^{\prime}, 4^{\prime}, 5^{\prime} \text { - } \\
\text { tetramethoxyflavone }\end{array}$ & Chukrasia $^{135}$ & $\begin{array}{l}\text { 5, 7-dihydroxy-3, 6, 8, 4'- } \\
\text { tetramethoxyflavone }\end{array}$ & Micromelum $^{94}$ \\
\hline $\begin{array}{l}\text { 5, 7-dihydroxy-3, 6, 3', 4'- } \\
\text { tetramethoxyflavone }\end{array}$ & Citrus $^{136}$, Gardenia $^{137}$ & $\begin{array}{l}\text { 5, 7-dihydroxy-3, 3', 4', 5'- } \\
\text { tetramethoxyflavone }\end{array}$ & Bridelia $^{138}$ \\
\hline $\begin{array}{l}\text { 5, 7-dihydroxy-2', 3', 4', 5'- } \\
\text { tetramethoxyflavone }\end{array}$ & Gardenia $^{139}$, Psiadia $^{140}$ & $\begin{array}{l}\text { 5, 7-dihydroxy- } 6,3^{\prime}, 4^{\prime}, 5^{\prime} \text { - } \\
\text { tetramethoxyflavone }\end{array}$ & Seriphidium ${ }^{141}$ \\
\hline $\begin{array}{l}\text { 5, 7-dihydroxy-8, 3', 4', 5'- } \\
\text { tetramethoxyflavone }\end{array}$ & Bracteantha $^{142}$ & $\begin{array}{l}\text { 5, 7-dihydroxy-8, 2', 3', 6'- } \\
\text { tetramethoxyflavone }\end{array}$ & Scutellaria $^{143}$ \\
\hline
\end{tabular}


5, 8-dihydroxy-6, 7, 3', 4'-

tetramethoxyflavone

5, 2'-dihydroxy-3, 7, 4', 5'-

tetramethoxyflavone (chrysosplenol

E)

5, 3'-dihydroxy-3, 6, 7, 4'-

tetramethoxyflavone (casticin or

vitexicarpin)

5, 3'-dihydroxy-6, 7, 8, 4'-

tetramethoxyflavone (gardenin D)

5, 4'-dihydroxy-7, 8, 2', 3'-

tetramethoxyflavone

5, 4'-dihydroxy-3, 6, 8, 3'-

tetramethoxyflavone

5, 4'-dihydroxy-7, 2', 3', 5'-

tetramethoxyflavone

5, 4'-dihydroxy-3, 7, 8, 3'-

tetramethoxyflavone

(Ternatin)

5, 4'-dihydroxy-3, 7, 8, 2'-

tetramethoxyflavone

5, 5'-dihydroxy-3, 7, 3', 4'-

tetramethoxyflavone

Gardenia $^{103,167}$

5, 5'-dihydroxy-6, 7, 2', 3'-

tetramethoxyflavone

6, 3'-hydroxy-3, 5, 7, 4'-

tetramethoxyflavone

Euryops $^{116}$

Artemisia $^{149}$,

Gutierrezia $^{150}$

Gardenia $^{110}$

Psiadia $^{140}$

Notholaena ${ }^{165}$

Gardenia $^{170}$

Jasonia $^{172}$
Chrysosplenium ${ }^{145}$

Citrus $^{147}$, Vitex ${ }^{148}$,

Gymnosperma $a^{158}$

Citrus $^{95}$, Egletes ${ }^{162}$

5, 4'-dihydroxy-3, 6, 7, 2'-

tetramethoxyflavone (Chrysosplin)

5, 5'-dihydroxy-3, 7, 2', 4'-

tetramethoxyflavone

(Chrysosplenol G)

6, 2'-hydroxy-5, 7, 8, 6'-

tetramethoxyflavone

6, 5'-hydroxy-3, 5, 7, 2'-

tetramethoxyflavone

5, 2'-dihydroxy-6, 7, 8, 6'-

tetramethoxyflavone

(Skullcapflavone II)

5, 3'-dihydroxy-6, 7, 4', 5'-

\section{5, 3'-dihydroxy-7, 8, 4', 5'-}

tetramethoxyflavone

5, 4'-dihydroxy-3, 6, 7, 3'-

B)

tetramethoxyflavone

5, 4'-dihydroxy-3, 7, 3', 5'-

tetramethoxyflavone
5, 2'-dihydroxy-7, 3', 4', 5'-

Gardenia $^{144}$

Scutellaria ${ }^{146}$

Murraya $^{151}$, Gardenia ${ }^{144}$,

Psiadia $^{152}$

Murraya $^{79}$

Artemisia $^{153}, \quad$ Vitex $^{154}$,

tetramethoxyflavone (chrysosplenol Achillea ${ }^{155}, \quad$ Miliusa $^{156}$,

Inula $^{157}$

5, 4'-dihydroxy-3, 6, 7, 8- Dracocephalum ${ }^{159}$,

tetramethoxyflavone (Calycopterin) Gutierrezia ${ }^{150}$, Vitex ${ }^{154}$

5, 4'-dihydroxy-6, 7, 8, 3'- $\quad$ Citrus $^{160}$,

Ononis $^{99}$,

Helianthus ${ }^{161}$

Aeonium $^{163}$, Solanum ${ }^{164}$

Citrus $^{147}$,

Chrysosplenium ${ }^{166}$

Chrysosplenium ${ }^{168}$,

Chlorophora ${ }^{169}$

tetramethoxyflavone
Scutellaria ${ }^{171}$

Blumea $^{112}$ 
8, 3'-dihydroxy-6, 7, 4', 5'-

tetramethoxyflavone

$2^{\prime}, 5^{\prime}$ - dihydroxy-3, 7, 8, 4'-

tetramethoxyflavone

3, 5, 4'-trihydroxy-6, 7, 8, 3'-

tetramethoxyflavone

3, 5, 8-trihydroxy-6, 7, 3', 4'-

tetramethoxyflavone

5, 7, 3'-trihydroxy-3, 6, 8, 4'-

tetramethoxyflavone

5, 7, 3'-trihydroxy-3, 6, 4', 5'-

tetramethoxyflavone

tetramethoxyflavone

5, 7, 4'-trihydroxy-3, 6, 8, 3'-

tetramethoxyflavone

$5,7,5^{\prime}$-trihydroxy-3, 6, 3', 4'-

tetramethoxyflavone

Gardenia $^{103,167}$

5, 7, 5'-trihydroxy-3, 6, 2', 4'-

tetramethoxyflavone

Eupatorium $^{180}$

$5,2^{\prime}, 5^{\prime}$-trihydroxy-6, 7, 3', 4'-

tetramethoxyflavone

Gardenia $^{144}$

5, 3', 5'-trihydroxy-3, 6, 7, 4'-

tetramethoxyflavone

Gardenia $^{170}$

$5,3^{\prime}, 5^{\prime}$-trihydroxy-5, 2', 3', 4'-

tetramethoxyflavone

Casimiroa $^{86}$

$5,6,3^{\prime}, 5^{\prime}$-tetrahydroxy-3, 7, 8, 4'-

tetramethoxyflavone

Gutierrezia $^{150}$

$5,7,2^{\prime}, 4^{\prime}$-tetrahydroxy-3, 6, 8, 5'- $\quad$ Gymnosperma ${ }^{158}$, $8,3^{\prime}$-dihydroxy-5, 6, 7, 4'-

tetramethoxyflavone

Vernonia $^{173}$

3, 5, 3'-trihydroxy-6, 7, 8, 4'-

tetramethoxyflavone

Zieridium $^{174}$

3, 5, 4'-trihydroxy-6, 7, 2, 5'-

tetramethoxyflavone

Celastrus $^{127}$

3, 5, 3'-trihydroxy-6, 7, 4', 5'-

tetramethoxyflavone

Premna ${ }^{176}$

5, 7, 2'-trihydroxy-3, 6, 4', 5'-

tetramethoxyflavone

Gutierrezia $^{177}$

5, 7, 3'-trihydroxy-3, 8, 4', 5'-

tetramethoxyflavone

Microglossa $^{178}$

5, 7, 4'-trihydroxy-3, 8, 3', 5'-

tetramethoxyflavone

Microglossa $^{178}$

5, 2', 4'-trihydroxy-6, 7, 8, 5'-

tetramethoxyflavone

Agecorynin D

Ageratum $^{179}$, ononis ${ }^{99}$,

Gutierrezia $^{150}$

$5,3^{\prime}, 4^{\prime}$-trihydroxy-3, 6, 7, 8-

tetramethoxyflavone

Gutierrezia $^{150}$

$5,2^{\prime}, 5^{\prime}$-trihydroxy-3, 6, 7, 4'- $\quad$ Inula $^{157}$,

tetramethoxyflavone

Chrysosplenium ${ }^{181}$

$5,3^{\prime}, 5^{\prime}$-trihydroxy-6, 7, 8, 4'-

tetramethoxyflavone (gardenin E)

Gardenia $^{110,111}$

$5,4^{\prime}, 6^{\prime}$-trihydroxy-6, 7, 2', 3'-

tetramethoxyflavone

Notholaena ${ }^{182}$

$5,7,4^{\prime}, 5^{\prime}$-tetrahydroxy-3, 6, 8, 2'-

tetramethoxyflavone

Gymnosperma ${ }^{158}$

$5,7,3^{\prime}, 4^{\prime}$-tetrahydroxy-3, 6, 8, 5'- Gutierrezia ${ }^{150}$ 


$\begin{array}{llll}\text { tetramethoxyflavone } & \text { Gutierrezia }^{177} & \text { tetramethoxyflavone } & \\ 5,7,2^{\prime}, 5^{\prime} \text {-tetrahydroxy-3,6,8, } 4^{\prime}- & & 5,7,3^{\prime}, 5^{\prime} \text {-tetrahydroxy-3, 6, 8, 4'- } & \text { Gymnosperma } \\ \text { tetramethoxyflavone } & \text { Gutierrezia }^{177} & \text { tetramethoxyflavone } & \text { Gutierrezia }\end{array}$

\section{$3,5,7,3^{\prime}, 4^{\prime}$-pentamethoxyflavone} (PMF 9)

Citrus $^{91}$, Callicarpa ${ }^{62}$,

Coix $^{42}$, Kaempferia ${ }^{183}, \quad 3,5,6,7,4^{\prime}$-pentamethoxyflavone $\quad$ Citrus $^{185}$, Varthemia ${ }^{120}$

Calophyllum ${ }^{184}$

$3,6,7,3^{\prime}, 4^{\prime}$-pentamethoxyflavone $\quad$ Oxalis ${ }^{186} \quad 3,5,7,8,4^{\prime}$-pentamethoxyflavone

Micromelum $^{46}$,

Tupistra $^{187}$

$5,6,7,3^{\prime}, 4^{\prime}$-pentamethoxyflavone $\quad$ Citrus $^{188}$ Orthosiphon $^{68}$, (sinensetin)

Murraya $^{80}$, Siraitia $^{189}$

5, 6, 2', 3', 4'-pentamethoxyflavone Ardisia $^{65}$, Casimiroa ${ }^{190}$

$5,6,3^{\prime}, 4^{\prime}, 5^{\prime}$-pentamethoxyflavone (cerrosilin B)

Casimiroa $^{191}$, Sargentia ${ }^{192} \quad 5,6,2^{\prime}, 3^{\prime}, 6^{\prime}$-pentamethoxyflavone Casimiroa $^{64}$ $5,6,7,8,4$ '-pentamethoxyflavone (tangeretin)
Citrus $^{41}$, Coix $^{42}$
$5,6,8,2^{\prime}, 5^{\prime}$-pentamethoxyflavone
Citrus ${ }^{193}$

Citrus $^{95}$, Murraya ${ }^{79,80}$,
$5,7,3^{\prime}, 4^{\prime}, 5^{\prime}$-pentamethoxyflavone
Neoraputia ${ }^{194}$,
5, 7, 2', 4', 6'-pentamethoxyflavone
Pleioblastus $^{195}$
Pleioblastus ${ }^{195}$
5, 7, 2', 3', 4'-pentamethoxyflavone
Andrographis ${ }^{196,197}$
$5,7,8,2^{\prime}, 4^{\prime}$-pentamethoxyflavone
Limnophila ${ }^{107}$
$5,7,8,3^{\prime}, 4^{\prime}$-pentamethoxyflavone (isosinensetin)
Citrus $^{41,89}$
$6,7,8,3^{\prime}, 4^{\prime}$-pentamethoxyflavone
Citrus $^{51}$

3-hydroxy-5, 7, 3', 4', 5'pentamethoxyflavone (PMF 11)

Murraya ${ }^{198}$

3-hydroxy-5, 6, 7, 8, 4'-

5-hydroxy-6, 7, 8, 2', 4'-

pentamethoxyflavone

Artemisia $^{200,201}$

pentamethoxyflavone

Citrus $^{199}$

3-hydroxy-6, 7, 8, 3', 4'-

5-hydroxy-6, 7, 8, 3', 4'-

pentamethoxyflavone

Citrus $^{203}$

5-hydroxy-3, 6, 7, 8, 4'-

Micromelum ${ }^{94}$,

pentamethoxyflavone

Euryops ${ }^{116}$

pentamethoxyflavone

Blumea $^{202}$

5-hydroxy-3, 7, 8, 3', 4'-

Citrus $^{76}$, Micromelum ${ }^{94}$

5-hydroxy-3, 7, 8, 2', 4'-

pentamethoxyflavone

Notholaena ${ }^{165}$

5-hydroxy-6, 7, 8, 2', 6'-

pentamethoxyflavone

Scutellaria ${ }^{204}$

5-hydroxy-3, 6, 7, 2', 5'-

Inula ${ }^{205}$ 
pentamethoxyflavone

5-hydroxy-3, 7, 3', 4', 5'-

pentamethoxyflavone (PMF 10)
Rhodomyrtus ${ }^{206}$,

Aeonium ${ }^{163}$,

Cassipourea $^{34}$,

Bosistoa $^{207}$

\section{Citrus $^{41,97}$, Murraya ${ }^{79}, 151, \quad 5$-hydroxy-3, 6, 8, 3', 4'-}

Capparis $^{210}$

Gardenia $^{139}$, Psiadia $^{140}$

pentamethoxyflavone

5-hydroxy-7, 8, 2', 3', 4'-

pentamethoxyflavone

6-hydroxy-3, 5, 7, 2', 5'-

pentamethoxyflavone

7-hydroxy-3, 5, 6, 3', 4'-

pentamethoxyflavone

7-hydroxy-5, 6, 3', 4', 5'-

pentamethoxyflavone

8-hydroxy-3, 5, 6, 7, 4'-

pentamethoxyflavone

4'-hydroxy-5, 6, 7, 3', 5'-

pentamethoxyflavone

4'-hydroxy-3, 5, 6, 7, 3'-

pentamethoxyflavone

3, 5-dihydroxy-6, 7, 8, 3', 4'-

pentamethoxyflavone

5, 7-dihydroxy-2', 3', 4', 5', 6'-

pentamethoxyflavone

5, 7-dihydroxy-3, 6, 8, 3', 4'-

pentamethoxyflavone

pentamethoxyflavone

5-hydroxy-6, 7, 3', 4', 5'-

pentamethoxyflavone

5-hydroxy-6, 7, 2', 4', 5'-

Ageratum ${ }^{73}$

Blumea $^{112}$

5-hydroxy-7, 8, 3', 4', 5'-

pentamethoxyflavone

6-hydroxy-5, 7, 8, 3', 4'-

Citrus $^{213,214}$

pentamethoxyflavone

7-hydroxy-3, 5, 6, 8, 4'-

Smilax $^{216}$

pentamethoxyflavone

7-hydroxy-5, 6, 8, 3', 4'-

Citrus $^{218}$

pentamethoxyflavone

3'-hydroxy-5, 6, 7, 8, 4'-

Ageratum $^{219,} 220$

pentamethoxyflavone

4'-hydroxy-5, 6, 7, 8, 3'-

Citrus $^{89}$

pentamethoxyflavone

5'-hydroxy-3, 5, 7, 2', 4'-

Zieridium $^{174}$

pentamethoxyflavone

Gardenia $^{139}$

pentamethoxyflavone

pentamethoxyflavone pentamethoxyflavone

5-hydroxy-3, 6, 7, 3' 4' $^{\prime}-\quad$ Citrus $^{97}, \quad$ Artemisia $^{153}$,

pentamethoxyflavone (artemetin) Vitex ${ }^{208}$, Ficus ${ }^{209}$

Gymnosperma ${ }^{158}$

Murraya $^{79,151}$, Lantana ${ }^{211}$,

Cardiospermum $^{212}$

Ageratum $^{121}$

pentamethylflavone (Agehoustin G)

Euryops $^{116}$

Citrus $^{215}$

Citrus $^{217}$

5, 6-dihydroxy-3, 7, 8, 3', 4'-

5, 7-dihydroxy-3, 6, 3', 4', 5'-

Melicope $^{223}$
Ostodes $^{222}$

\section{Citrus $^{98}$}

Citrus $^{89,}$ 98, 199

Citrus $^{221}$

Distemonanthus ${ }^{114}$

Ostodes22

Gardenia $^{103,167}$ 
5, 2'-dihydroxy-6, 7, 8, 4', 5'-

pentamethoxyflavone Ageratum ${ }^{224}$

Agehoustin F

5, 3'-dihydroxy-6, 7, 8, 4', 5'-

pentamethoxyflavone

5, 5'-dihydroxy-3, 6, 7, 3', 4'-

pentamethoxyflavone

6, 3'-dihydroxy-3, 5, 7, 4', 5'-

pentamethoxyflavone

$3,5,3^{\prime}$-trihydroxy- $6,7,8,4^{\prime}, 5^{\prime}$ -

pentamethoxyflavone

$5,7,3^{\prime}$-trihydroxy-3, 6, 8, 4', 5'-

pentamethoxyflavone

$5,7,5^{\prime}$-trihydroxy-3, $6,8,2^{\prime}, 4^{\prime}-$
pentamethoxyflavone

$5,4^{\prime}, 5^{\prime}$-trihydroxy-3, 6, 7, 8, 2'-

pentamethoxyflavone

Gymnosperma ${ }^{158}$

$3,5,6,7,3^{\prime}, 4^{\prime}$ - hexamethylflavone $\quad$ Citrus $^{185}$

$3,5,7,8,2^{\prime}, 5^{\prime}$ - hexamethylflavone

$3,5,7,3^{\prime}, 4^{\prime}, 5^{\prime}$-hexamethylflavone

(PMF 12)

Citrus $^{232}$

Myrcia $^{233}$, Murraya ${ }^{234}$,

Ficus ${ }^{209}$

$3,5,7,8,3^{\prime}, 4^{\prime}$-hexamethylflavone

$5,7,4^{\prime}$-trihydroxy-3, 6, 8, 2', 5'-

pentamethoxyflavone

$5,3^{\prime}, 5^{\prime}$-trihydroxy-3, 6, 7, 8, 4'-

pentamethoxyflavone

$3,5,6,7,8,4^{\prime}$ - hexamethylflavone $\quad$ Citrus $^{230}$, varthemia ${ }^{231}$

$3,6,7,8,2^{\prime}, 5^{\prime}$ - hexamethylflavone Citrus $^{87}$

$5,6,7,2^{\prime}, 4^{\prime}, 5^{\prime}$-hexamethylflavone $\quad$ Ageratum $^{12}$

$5,6,7,3^{\prime}, 4^{\prime}, 5^{\prime}$-hexamethylflavone

Citrus $^{98}$, Murraya ${ }^{79,}{ }^{151}$,

Ageratum $^{220}$, Lantana ${ }^{211}$,

Eremophila ${ }^{236}$

$5,6,2^{\prime}, 3^{\prime}, 4^{\prime}, 6^{\prime}$-hexamethylflavone Casimiroa ${ }^{237}$

$5,2^{\prime}, 3^{\prime}, 4^{\prime}, 5^{\prime}, 6^{\prime}$-hexamethylflavone Casimiroa $^{86}$

$5,7,8,3^{\prime}, 4^{\prime}, 5^{\prime}$-hexamethylflavone

3-hydroxy 5, 6, 7, 8, 3', 4'- $5,6,2^{\prime}, 3^{\prime}, 5^{\prime}, 6^{\prime}$-hexamethylflavone

$5,7,2^{\prime}, 3^{\prime}, 4^{\prime}, 5^{\prime}$-hexamethylflavone

$5,7,8,2^{\prime}, 3^{\prime}, 4^{\prime}$-hexamethylflavone

$5,6,7,8,3^{\prime}, 4^{\prime}$-hexamethylflavone
Casimiroa $^{64,237}$

Capparis $^{210}$

Stenoloma ${ }^{238}$

Citrus $^{41}$, Ageratum ${ }^{220}$ 
hexamethylflavone (Natsudaidain)

5-hydroxy-6, 7, 8, 2', 4', 5'-

hexamethylflavone

Ageratum $^{224}$

Agehoustin E

5-hydroxy-3, 7, 8, 3', 4', 5'-

hexamethylflavone

5-hydroxy-7, 2', 3', 4', 5', 6'-

hexamethylflavone

5-hydroxy-7, 8, 2', 3', 4', 5'-

hexamethylflavone (isoagecorynin F)

7-hydroxy-3, 5, 6, 8, 3', 4'-

hexamethylflavone

8-hydroxy-3, 5, 7, 3', 4', 5'-

hexamethylflavone

3'-hydroxy-5, 6, 7, 2', 4', 5'-

hexamethylflavone (agecorynin G)

3, 5-dihydroxy-6, 7, 8, 3', 4', 5'-

hexamethylflavone

5, 2'-dihydroxy-3, 6, 7, 8, 4', 5'-

hexamethylflavone

5, 3'-dihydroxy-3, 6, 7, 8, 4', 5'-

hexamethylflavone

5, 7-dihydroxy-3, 6, 8, 2', 4', 5'-

hexamethylflavone

$3,5,7,8,3^{\prime}, 4^{\prime}, 5^{\prime}-$

heptamethoxyflavone

$5,6,7,8,3^{\prime}, 4^{\prime}, 5^{\prime}-$

heptamethoxyflavone
Gymnosperma ${ }^{158}$,

Gutierrezia $^{150}$

Gutierrezia $^{177}$

Gymnosperma ${ }^{158}$

Murraya $^{239}$

Gardenia $^{139}$

Ageratum $^{121}$

Citrus $^{214}$

Murraya $^{239}$

Ageratum $^{121}$

Athrixia $^{241}$

Citrus $^{243,244}$, Murraya ${ }^{239}$

Murraya $^{79}$, Hedyotis,

Ageratum $^{220}$, Blumea $^{245}$
(Nobiletin)

5-hydroxy-3, 6, 7, 8, 3', 4'-

hexamethylflavone

Citrus $^{97}$, Vitex $^{154}$

5-hydroxy-6, 7, 8, 3', 4', 5'-

Citrus $^{98}$, Gardenia ${ }^{110,}{ }^{111}$,

hexamethylflavone (gardenin A) Murraya ${ }^{79,151}$

5-hydroxy-3, 6, 7, 3', 4', 5'-

hexamethylflavone

Cleome $^{226}$

5-hydroxy-6, 7, 2', 3', 4', 5'-

hexamethylflavone (agecorynin F)

Ageratum $^{121}$

6-hydroxy-3, 5, 7, 8, 3', 4'-

hexamethylflavone

Citrus $^{240}$

8-hydroxy-3, 5, 6, 7, 3', 4'-

hexamethylflavone

Citrus $^{218}$

8-hydroxy-5, 6, 7, 3', 4', 5'-

hexamethylflavone

Citrus $^{98}$

3'-hydroxy-5, 6, 7, 8, 4', 5'-

hexamethylflavone

Ageratum $^{220}$

4'-hydroxy-3, 5, 6, 7, 3', 5'-

hexamethylflavone

Murraya $^{242}$

5, 3'-dihydroxy-6, 7, 8, 2', 4', 5'-

hexamethylflavone

Ageratum $^{121}$

(Agehoustin D)

5, 7-dihydroxy-3, 6, 8, 3', 4', 5'-

hexamethylflavone

Athrixia $^{227}$, Gutierrezia ${ }^{150}$

$3,5,6,7,8,3^{\prime}, 4^{\prime}-$

heptamethoxyflavone

Citrus $^{41}$, Coix $^{42}$

$3,5,6,7,3^{\prime}, 4^{\prime}, 5^{\prime}-$

heptamethoxyflavone 


\begin{tabular}{|c|c|c|c|}
\hline $\begin{array}{l}5,6,7,8,2^{\prime}, 4^{\prime}, 5^{\prime}- \\
\text { heptamethylflavone (agecorynin C) }\end{array}$ & Ageratum $^{121,179}$ & $\begin{array}{l}5,6,7,2^{\prime}, 3^{\prime}, 4^{\prime}, 5^{\prime}- \\
\text { heptamethylflavone (Agehoustin B) }\end{array}$ & Ageratum $^{121}$ \\
\hline $\begin{array}{l}\text { 5-hydroxy-3, 6, 7, 8, 3', 4', 5'- } \\
\text { heptamethylflavone }\end{array}$ & Gutierrezia $^{150}$ & $\begin{array}{l}5,7,8,2^{\prime}, 3^{\prime}, 4^{\prime}, 5^{\prime}- \\
\text { heptamethylflavone }\end{array}$ & Ageratum $^{121}$ \\
\hline $\begin{array}{l}3,5,6,7,8,3^{\prime}, 4^{\prime}, 5^{\prime}- \\
\text { octamethoxyflavone (exoticin) }\end{array}$ & Nicotiana $^{246}$, Murraya $^{234}$ & $\begin{array}{l}\text { 3'-hydroxy-5, 6, 7, 8, 2', 4', 5'- } \\
\text { heptamethylflavone (Agehoustin C) }\end{array}$ & Ageratum 121 \\
\hline $\begin{array}{l}5,6,7,8,2^{\prime}, 3^{\prime}, 4^{\prime}, 5^{\prime}- \\
\text { octamethoxyflavone (Agehoustin A) }\end{array}$ & Ageratum 121 & $\begin{array}{l}3,5,6,7,8,2^{\prime}, 4^{\prime}, 5^{\prime}- \\
\text { octamethoxyflavone }\end{array}$ & Pogostemon ${ }^{247}$ \\
\hline $\begin{array}{l}3^{\prime}, 4^{\prime}, 6,7 \text {-bismethylenedioxy-3, 5, 8- } \\
\text { dimethoxyflavone }\end{array}$ & Melicope $^{248}$ & $\begin{array}{l}\text { 3', 4', 6, 7-bismethylenedioxy-3, 5- } \\
\text { dimethoxyflavone }\end{array}$ & Melicope $^{248}$ \\
\hline $\begin{array}{l}\text { 3', 4'-methylenedioxy-5, 6, 7- } \\
\text { trimethoxyflavone }\end{array}$ & $\begin{array}{l}\text { Ageratum }^{121} \\
\text { Neoraputia }^{194}\end{array}$ & $\begin{array}{l}\text { 3', 4'-methylenedioxy-3, 5, 7- } \\
\text { trimethoxyflavone }\end{array}$ & $\begin{array}{l}\text { Melicope }^{248} \\
\text { Goniothalamus } \\
249\end{array}$ \\
\hline $\begin{array}{l}6,7,4^{\prime}, 5^{\prime} \text {-dimethylenedioxy-3, 5, 3'- } \\
\text { trimethoxyflavone }\end{array}$ & Nicotiana $^{246}$ & $\begin{array}{l}\text { 3', 4'-methylenedioxy-5, 7, 5'- } \\
\text { trimethoxyflavone }\end{array}$ & $\operatorname{Piper}^{250}$, Bauhinia ${ }^{251}$ \\
\hline $\begin{array}{l}3^{\prime}, 4^{\prime} \text {-methylenedioxy-5, 6, 7, 5'- } \\
\text { tetramethoxyflavone }\end{array}$ & $\begin{array}{l}\text { Bauhinia }^{251} \text {, Ageratum } \\
\text { Entada }^{252}, \text { Neoraputia }^{194}\end{array}$ & $\begin{array}{l}3^{\prime}, 4^{\prime} \text {-methylenedioxy-5, 6, 2', 5'- } \\
\text { tetramethoxyflavone }\end{array}$ & Struthiola $^{63}$ \\
\hline $\begin{array}{l}\text { 3', 4'-methylenedioxy-3, 5, 6, 7- } \\
\text { tetramethoxyflavone }\end{array}$ & Melicope 248,249 & $\begin{array}{l}3^{\prime}, 4^{\prime} \text {-methylenedioxy-5, 6, 7, 8- } \\
\text { tetramethoxyflavone }\end{array}$ & Ageratum $^{121,220}$ \\
\hline $\begin{array}{l}3^{\prime}, 4^{\prime} \text {-methylenedioxy-3, 5, 6, 7, 8, - } \\
\text { pentamethoxyflavone }\end{array}$ & Melicope $^{248}$ & $\begin{array}{l}\text { 7-hydroxy-3', 4'-methylenedioxy-5, } \\
6,8,5^{\prime} \text {-tetramethoxyflavone }\end{array}$ & Ageratum 220 \\
\hline $\begin{array}{l}3^{\prime}, 4^{\prime} \text {-methylenedioxy-5, 6, 2', 5', 6'- } \\
\text { pentamethoxyflavone }\end{array}$ & Struthiola $^{63}$ & $\begin{array}{l}\text { 3', 4'-methylenedioxy-5, 6, 7, 8, 5'- } \\
\text { pentamethoxyflavone (eupalestin) }\end{array}$ & $\begin{array}{l}\text { Eupatorium }^{253} \text {, } \\
\text { Ageratum }^{179} \\
\text { Colebrookea }^{254}\end{array}$ \\
\hline $\begin{array}{l}\text { 4', 5'-methylenedioxy- } 3,5,6,7,8, \\
\text { 3'-hexamethoxyflavone }\end{array}$ & Nicotiana $^{246}$ & $\begin{array}{l}\text { 6, 7-methylenedioxy-3, 5, 8, 3', 4', } \\
\text { 5'-hexamethoxyflavone }\end{array}$ & Nicotiana $^{246}$ \\
\hline
\end{tabular}

PMFs evaluated in our study were in bold.

\section{References}

(1) Nguyen, P. T.; Luyen, B. T. T.; Kim, J. H.; Jo, A. R.; Yang, S. Y.; Nguyen, T. D.; Minh, C. V.; 
Kim, Y. H., Soluble epoxide hydrolase inhibitory activity by rhizomes of Kaempferia parviflora Wall. ex Baker. Medicinal Chemistry Research 2016, 25, (4), 704-711.

(2) Jung, H.; Shin, S. Y.; Jung, Y.; Tran, T. A.; Lee, H. O.; Jung, K.; Koh, D.; Cho, S. K.; Lim, Y., Quantitative Relationships Between the Cytotoxicity of Flavonoids on the Human Breast Cancer StemLike Cells MCF7-SC and Their Structural Properties. Chem. Biol. Drug Des. 2015, 86, (4), 496-508.

(3) Sae-Wong, C.; Matsuda, H.; Tewtrakul, S.; Tansakul, P.; Nakamura, S.; Nomura, Y.; Yoshikawa, M., Suppressive effects of methoxyflavonoids isolated from Kaempferia parviflora on inducible nitric oxide synthase (iNOS) expression in RAW 264.7 cells. J. Ethnopharmacol. 2011, 136, (3), 488-495.

(4) Tewtrakul, S.; Subhadhirasakul, S.; Karalai, C.; Ponglimanont, C.; Cheenpracha, S., Antiinflammatory effects of compounds from Kaempferia parviflora and Boesenbergia pandurata. Food Chem. 2009, 115, (2), 534-538.

(5) Azuma, T.; Kayano, S.; Matsumura, Y.; Konishi, Y.; Tanaka, Y.; Kikuzaki, H., Antimutagenic and a -glucosidase inhibitory effects of constituents from Kaempferia parviflora. Food Chem. 2010, $125,(2), 471-475$.

(6) Du, Q.; Chen, H., The methoxyflavones in Citrus reticulata Blanco cv. ponkan and their antiproliferative activity against cancer cells. Food Chem. 2009, 119, (2), 567-572.

(7) Pereira, C. V.; Duarte, M.; Silva, P.; Da Silva, A. B.; Duarte, C. M. M.; Cifuentes, A.; GarciaCanas, V.; Bronze, M. R.; Albuquerque, C.; Serra, A. T., Polymethoxylated flavones target cancer stemness and improve the antiproliferative effect of 5-fluorouracil in a 3D cell model of colorectal cancer. Nutrients 2019, 11, (2), 326.

(8) Ju, H. M.; Yeon, J. J.; Jung, K. E.; Hyun, A. J.; Young, C. H.; Hyun, C.; Lee, N. H., Tetramethylo-scutellarin isolated from peels of immature shiranuhi fruit exhibits anti-inflammatory effects on lpsinduced raw264.7 cells. Trop. J. Pharm. Res. 2017, 16, (9), 2197-2205.

(9) Pandith, H.; Zhang, X.; Thongpraditchote, S.; Wongkrajang, Y.; Gritsanapan, W.; Baek, S. J., Effect of Siam weed extract and its bioactive component scutellarein tetramethyl ether on antiinflammatory activity through NF- к B pathway. J. Ethnopharmacol. 2013, 147, (2), 434-441.

(10) Li, X.; Zhang, C.; Guo, S.; Rajaram, P.; Lee, M.; Chen, G.; Fong, R.; Gonzalez, A.; Zhang, Q.; Zheng, S.; Wang, G.; Chen, Q., Structure-activity relationship and pharmacokinetic studies of 3-Osubstituted flavonols as anti-prostate cancer agents. Eur. J. Med. Chem. 2018, 157, 978-993.

(11) Wen, R.; Lv, H.; Jiang, Y.; Tu, P., Anti-inflammatory flavone and chalcone derivatives from the 
roots of Pongamia pinnata (L.) Pierre. Phytochemistry (Elsevier) 2018, 149, 56-63.

(12) Ranga Rao, R.; Tiwari, A. K.; Prabhakar Reddy, P.; Suresh Babu, K.; Ali, A. Z.; Madhusudana, K.; Madhusudana Rao, J., New furanoflavanoids, intestinal a -glucosidase inhibitory and free-radical (DPPH) scavenging, activity from antihyperglycemic root extract of Derris indica (Lam.). Bioorgan. Med. Chem. 2009, 17, (14), 5170-5175.

(13) Weng, Z.; Patel, A. B.; Panagiotidou, S.; Theoharides, T. C., The novel flavone tetramethoxyluteolin is a potent inhibitor of human mast cells. J. Allergy Clin. Immun. 2015, 135, (4), 1044-1052.e5.

(14) Taracanova, A.; Tsilioni, I.; Conti, P.; Norwitz, E. R.; Leeman, S. E.; Theoharides, T. C., Substance P and IL-33 administered together stimulate a marked secretion of IL-1 $\beta$ from human mast cells, inhibited by methoxyluteolin. P. Natl. Acad. Sci. Usa. 2018, 115, (40), E9381-E9390.

(15) Azuma, T.; Kayano, S.; Matsumura, Y.; Konishi, Y.; Tanaka, Y.; Kikuzaki, H., Antimutagenic and a -glucosidase inhibitory effects of constituents from Kaempferia parviflora. Food Chem. 2010, $125,(2), 471-475$.

(16) Yuan, X.; Li, L.; Shi, W.; Liu, H.; Huang, X.; Liu, Z.; Wu, L., TMF protects chondrocytes from ER stress-induced apoptosis by down-regulating GSK-3 $\beta$. Biomed. Pharmacother. 2017, 89, 1262-1268.

(17) Ravishankar, D.; Watson, K. A.; Boateng, S. Y.; Green, R. J.; Greco, F.; Osborn, H. M. I., Exploring quercetin and luteolin derivatives as antiangiogenic agents. Eur. J. Med. Chem. 2015, 97, 259274.

(18) Juvale, K.; Stefan, K.; Wiese, M., Synthesis and biological evaluation of flavones and benzoflavones as inhibitors of BCRP/ABCG2. Eur. J. Med. Chem. 2013, 67, 115-126.

(19) Gallus, J.; Juvale, K.; Wiese, M., Characterization of 3-methoxy flavones for their interaction with ABCG2 as suggested by ATPase activity. Biochimica et Biophysica Acta, Biomembranes 2014, 1838, (11), 2929-2938.

(20) Ikegawa, T.; Ohtani, H.; Koyabu, N.; Juichi, M.; Iwase, Y.; Ito, C.; Furukawa, H.; Naito, M.; Tsuruo, T.; Sawada, Y., Inhibition of P-glycoprotein by flavonoid derivatives in adriamycin-resistant human myelogenous leukemia (K562/ADM) cells. Cancer Letters (Shannon, Ireland) 2002, 177, (1), 89-93.

(21) Ji, X.; Melman, N.; Jacobson, K. A., Interactions of Flavonoids and Other Phytochemicals with Adenosine Receptors. J. Med. Chem. 1996, 39, (3), 781-8. 
(22) Tewtrakul, S.; Subhadhirasakul, S.; Karalai, C.; Ponglimanont, C.; Cheenpracha, S., Antiinflammatory effects of compounds from Kaempferia parviflora and Boesenbergia pandurata. Food Chem. 2009, 115, (2), 534-538.

(23) Martins, I. L.; Charneira, C.; Gandin, V.; Ferreira Da Silva, J. L.; Justino, G. C.; Telo, J. P.; Vieira, A. J. S. C.; Marzano, C.; Antunes, A. M. M., Selenium-Containing Chrysin and Quercetin Derivatives: Attractive Scaffolds for Cancer Therapy. J. Med. Chem. 2015, 58, (10), 4250-4265.

(24) Yuan, J.; Wong, I. L. K.; Jiang, T.; Wang, S. W.; Liu, T.; Bin, J. W.; Chow, L. M. C.; Biao, W. S., Synthesis of methylated quercetin derivatives and their reversal activities on P-gp- and BCRPmediated multidrug resistance tumor cells. Eur. J. Med. Chem. 2012, 54, 413-422.

(25) Rajaram, P.; Jiang, Z.; Chen, G.; Rivera, A.; Phasakda, A.; Zhang, Q.; Zheng, S.; Wang, G.; Chen, Q., Nitrogen-containing derivatives of O-tetramethylquercetin: Synthesis and biological profiles in prostate cancer cell models. Bioorg. Chem. 2019, 87, 227-239.

(26) Han, Y.; Ding, Y.; Xie, D.; Hu, D.; Li, P.; Li, X.; Xue, W.; Jin, L.; Song, B., Design, synthesis, and antiviral activity of novel rutin derivatives containing 1,4-pentadien-3-one moiety. Eur. J. Med. Chem. 2015, 92, 732-737.

(27) Yuan, J.; Wong, I. L. K.; Jiang, T.; Wang, S. W.; Liu, T.; Bin, J. W.; Chow, L. M. C.; Biao, W. S., Synthesis of methylated quercetin derivatives and their reversal activities on P-gp- and BCRPmediated multidrug resistance tumor cells. Eur. J. Med. Chem. 2012, 54, 413-422.

(28) Du, J.; Wu, J.; Li, Z.; Zhang, C.; Shi, M.; Zhao, J.; Jin, M.; Liu, H., Pentamethylquercetin protects against cardiac remodeling via activation of Sestrin2. Biochem. Bioph. Res. Co. 2019, 512, (2), 412-420.

(29) Lesjak, M.; Beara, I.; Simin, N.; Pintac, D.; Majkic, T.; Bekvalac, K.; Orcic, D.; Mimica-Dukic, N., Antioxidant and anti-inflammatory activities of quercetin and its derivatives. J. Funct. Foods 2018, $40,68-75$.

(30) Han, Y.; Wu, J.; Shen, J.; Chen, L.; He, T.; Jin, M.; Liu, H., Pentamethylquercetin induces adipose browning and exerts beneficial effects in 3T3-L1 adipocytes and high-fat diet-fed mice. Sci. Rep.-UK 2017, 7, (1), 1-13.

(31) Youn, K.; Lee, J.; Ho, C.; Jun, M., Discovery of polymethoxyflavones from black ginger (Kaempferia parviflora) as potential $\beta$-secretase (BACE1) inhibitors. J. Funct. Foods 2016, 20, 567574.

(32) Jakhar, R.; Paul, S.; Park, Y. R.; Han, J.; Kang, S. C., 3,5,7,3',4'-Pentamethoxyflavone, a quercetin 
derivative protects DNA from oxidative challenges: Potential mechanism of action. Journal of Photochemistry and Photobiology, B: Biology 2014, 131, 96-103.

(33) Shen, J. Z.; Ma, L. N.; Han, Y.; Liu, J. X.; Yang, W. Q.; Chen, L.; Liu, Y.; Hu, Y.; Jin, M. W., Pentamethylquercetin generates beneficial effects in monosodium glutamate-induced obese mice and C2C12 myotubes by activating AMP-activated protein kinase. Diabetologia 2012, 55, (6), 1836-1846.

(34) Chaturvedula, V. S. P.; Norris, A.; Miller, J. S.; Ratovoson, F.; Andriantsiferana, R.; Rasamison, V. E.; Kingston, D. G. I., Cytotoxic Diterpenes from Cassipourea madagascariensis from the Madagascar Rainforest. J. Nat. Prod. 2006, 69, (2), 287-289.

(35) Zarmouh, N. O.; Messeha, S. S.; Mateeva, N.; Gangapuram, M.; Flowers, K.; Eyunni, S. V. K.; Zhang, W.; Redda, K. K.; Soliman, K. F. A., The antiproliferative effects of flavonoid MAO inhibitors on prostate cancer cells. Molecules 2020, 25, (9), 2257.

(36) Mateeva, N.; Gangapuram, M.; Mazzio, E.; Eyunni, S.; Soliman, K. F. A.; Redda, K. K., Biological evaluation of synthetic chalcone and flavone derivatives as anti-inflammatory agents. Medicinal Chemistry Research 2015, 24, (4), 1672-1680.

(37) Ohtani, H.; Ikegawa, T.; Honda, Y.; Kohyama, N.; Morimoto, S.; Shoyama, Y.; Juichi, M.; Naito, M.; Tsuruo, T.; Sawada, Y., Effects of various methoxyflavones on vincristine uptake and multidrug resistance to vincristine in P-gp-overexpressing K562/ADM cells. Pharm Res 2007, 24, (10), 1936-43.

(38) Matsuda, H.; Morikawa, T.; Yoshikawa, M., Antidiabetogenic constituents from several natural medicines. Pure Appl. Chem. 2002, 74, (7), 1301-1308.

(39) Burda, S.; Oleszek, W., Antioxidant and antiradical activities of flavonoids. J. Agr. Food Chem. 2001, 49, (6), 2774-2779.

(40) Liang, X.; Zhao, C.; Su, W., Multi-constituent determination and fingerprint analysis of Scutellaria indica L. using ultra high performance liquid chromatography coupled with quadrupole timeof-flight mass spectrometry. J. Sep. Sci. 2015, 38, (21), 3704-3711.

(41) Ke, Z.; Yang, Y.; Tan, S.; Zhou, Z., Characterization of Polymethoxylated Flavonoids in the Peels of Chinese Wild Mandarin (Citrus reticulataBlanco) by UPLC-Q-TOF-MS/MS. Food Anal. Method. 2017, 10, (5), 1328-1338.

(42) Chen, H. J.; Chung, C. P.; Chiang, W.; Lin, Y. L., Anti-inflammatory effects and chemical study of a flavonoid-enriched fraction from adlay bran. Food Chem. 2011, 126, (4), 1741-8.

(43) Miyata, Y.; Tatsuzaki, J.; Yang, J.; Kosano, H., Potential Therapeutic Agents, Polymethoxylated 
Flavones Isolated from Kaempferia parviflora for Cataract Prevention through Inhibition of Matrix Metalloproteinase-9 in Lens Epithelial Cells. Biol. Pharm. Bull. 2019, 42, (10), 1658-1664.

(44) Suradej, B.; Sookkhee, S.; Panyakaew, J.; Mungkornasawakul, P.; Wikan, N.; Smith, D. R.; Potikanond, S.; Nimlamool, W., Kaempferia parviflora extract inhibits STAT3 activation and interleukin-6 production in HeLa cervical cancer cells. Int. J. Mol. Sci. 2019, 20, (17), 4226/1-4226/21.

(45) Horigome, S.; Maeda, M.; Ho, H.; Shirakawa, H.; Komai, M., Effect of Kaempferia parviflora extract and its polymethoxyflavonoid components on testosterone production in mouse testis-derived tumour cells. J. Funct. Foods 2016, 26, 529-538.

(46) Vidar, W. S.; Macabeo, A. P. G.; Knorn, M.; Kohls, P.; Aguinaldo, A. M., Polymethoxylated flavones from Micromelum compressum. Biochemical Systematics \& Ecology 2013, 50, (Complete), 4850.

(47) Chan, Y.; Wang, C.; Hwang, T.; Juang, S.; Hung, H.; Kuo, P.; Chen, P.; Wu, T., The constituents of the stems of cissus assamica and their bioactivities. Molecules 2018, 23, (11), 2799/1-2799/10.

(48) Ponguschariyagul, S.; Sichaem, J.; Khumkratok, S.; Siripong, P.; Lugsanangarm, K.; Tip-pyang, S., Caloinophyllin A, a new chromanone derivative from Calophyllum inophyllum roots. Nat. Prod. Res. 2018, 32, (21), 2535-2541.

(49) He, K.; Zhang, S.; Li, X.; Fan, Y.; Jin, X., Chemical composition and free radicals restraining activity of extracts from three Manglietia species leaves. Journal of Forestry Research (Harbin, China) 2007, 18, (3), 193-198.

(50) Jasril; Mooi, L. Y.; Lajis, N. H.; Ali, A. M.; Sukari, M. A.; Rahman, A. A.; Othman, A. G.; Kikuzaki, H.; Nakatani, N., Antioxidant and antitumor promoting activities of the flavonoids from Hedychium thyrsiforme. Pharmaceutical Biology (Lisse, Netherlands) 2003, 41, (7), 506-511.

(51) Han, S.; Kim, H. M.; Lee, S., Simultaneous determination of polymethoxyflavones in Citrus species, Kiyomi tangor and Satsuma mandarin, by high performance liquid chromatography. Food Chem. 2012, 134, (2), 1220-4.

(52) Han, S.; Kim, H. M.; Lee, J. M.; Mok, S.; Lee, S., Isolation and Identification of Polymethoxyflavones from the Hybrid Citrus, Hallabong. J. Agr. Food Chem. 2010, 58, (17), 9488-9491. (53) Zhang, X.; Liang, C.; Li, C.; Bu, M.; Bu, L.; Xiao, Y.; Sun, H.; Zhang, L., Simultaneous Qualitative and Quantitative Study of Main Compounds in Commelina communis Linn. by UHPLC-QTOF-MS-MS and HPLC-ESI-MS-MS. J. Chromatogr. Sci. 2018, 56, (7), 582-594. 
(54) Wen, R.; Lv, H. N.; Jiang, Y.; Tu, P. F., Anti-inflammatory flavone and chalcone derivatives from the roots of Pongamia pinnata (L.) Pierre. Phytochemistry 2018, 149, 56-63.

(55) Yin, H.; Wu, J.; Nan, H.; Zhang, S., New prenylated flavones from Pongamia pinnata. Pharmazie 2006, 61, (1), 76-78.

(56) Anusiri, P.; Choodej, S.; Chumriang, P.; Adisakwattana, S.; Pudhom, K., Inhibitory effects of flavonoids from stem bark of Derris indica on the formation of advanced glycation end products. $J$. Ethnopharmacol. 2014, 158, (Part_A), 437-441.

(57) Saraphon, C.; Boonloh, K.; Kukongviriyapan, V.; Yenjai, C., Cytotoxic flavonoids from the fruits of Derris indica. J. Asian Nat. Prod. Res. 2017, 19, (12), 1198-1203.

(58) Tan, A.; Yang, H.; Li, Y.; Bi, Z.; Zhang, M.; Wang, Z., Study on the chemical constituents of Ligularia dictyoneura. Zhongguo Yaoxue Zazhi (Beijing, China) 2004, 39, (7), 498-499.

(59) Yoshioka, T.; Inokuchi, T.; Fujioka, S.; Kimura, Y., Phenolic compounds and flavonoids as plant growth regulators from fruit and leaf of Vitex rotundifolia. Z Naturforsch C J Biosci 2004, 59, (7-8), 509-14.

(60) Barakat, D. A., Insecticidal and antifeedant activities and chemical composition of Casimiroa edulis La Llave \& Lex (Rutaceae) leaf extract and its fractions against Spodoptera littoralis larvae. Australian Journal of Basic and Applied Sciences 2011, 5, (9), 693-703.

(61) Ubaldo-Suarez, D.; Estrada Reyes, R.; De la Rosa-Sierra, R.; Martinez-Vazquez, M., Antidepressant-like effect of Casimiroa pubescens root extracts. Nat. Prod. Res. 2019, 33, (17), 25262530.

(62) Xu, Y.; Ramirez-Ahumada, M. D. C.; Valeriote, F. A.; Gunatilaka, A. A. L., Solid tumor inhibitory and other constituents of Casimiroa tetrameria. Zhongguo Tianran Yaowu 2011, 9, (5), 334337.

(63) Ayers, S.; Zink, D. L.; Mohn, K.; Powell, J. S.; Brown, C. M.; Murphy, T.; Brand, R.; Pretorius, S.; Stevenson, D.; Thompson, D.; Singh, S. B., Flavones from Struthiola argentea with anthelmintic activity in vitro. Phytochemistry 2008, 69, (2), 541-545.

(64) Heneka, B.; Rimpler, H.; Ankli, A.; Sticher, O.; Gibbons, S.; Heinrich, M., A furanocoumarin and polymethoxylated flavonoids from the Yucatec Mayan plant Casimiroa tetrameria. Phytochemistry 2005, 66, (6), 649-52.

(65) Parveen, M.; Khan, N. U. D., Two new flavones from Ardisia floribunda Wall in Roxb. Indian 
Journal of Chemistry, Section B: Organic Chemistry Including Medicinal Chemistry 1987, 26B, (9), 8945.

(66) Arigò, A.; Rigano, F.; Micalizzi, G.; Dugo, P.; Mondello, L., Oxygen heterocyclic compound screening in Citrus essential oils by linear retention index approach applied to liquid chromatography coupled to photodiode array detector. Flavour Frag. J. 2019, 34, (5), 349-364.

(67) Yu, L.; Chen, M.; Liu, J.; Huang, X.; He, W.; Qing, Z.; Zeng, J., Systematic detection and identification of bioactive ingredients from Citrus aurantium L. var. amara using HPLC-Q-TOF-MS combined with a screening method. Molecules 2020, 25, (2), 357.

(68) Deipenbrock, M.; Hensel, A., Polymethoxylated flavones from Orthosiphon stamineus leaves as antiadhesive compounds against uropathogenic E. coli. Fitoterapia 2019, 139, 104387.

(69) Emani, L.; Ravada, S.; Meka, B.; Garaga, M.; Golakoti, T., A New Flavanone from the Leaves of Chromolaena odorata. Nat. Prod. Commun. 2015, 10, (9), $1934578 X 1501000918$.

(70) Zheng, G. D.; Zhou, P.; Yang, H.; Li, Y. S.; Li, P.; Liu, E. H., Rapid resolution liquid chromatography-electrospray ionisation tandem mass spectrometry method for identification of chemical constituents in Citri Reticulatae Pericarpium. Food Chem. 2013, 136, (2), 604-11.

(71) Tatum, J. H.; Berry, R. E., Six new flavonoids from Citrus. Phytochemistry 1972, 11, (7), 22832288.

(72) Zheng, G.; Yang, X.; Chen, B.; Chao, Y.; Hu, P.; Cai, Y.; Wu, B.; Wei, M., Identification and determination of chemical constituents of Citrus reticulata semen through ultra high performance liquid chromatography combined with Q Exactive Orbitrap tandem mass spectrometry. J. Sep. Sci. 2020, 43, (2), 438-451.

(73) Xu, C.; Wang, Z., Chemical constituents from roots of Andrographis paniculata. Yaoxue Xuebao 2011, 46, (3), 317-321.

(74) Wang, Y.; Jiao, J.; Yang, Y.; Yang, M.; Zheng, Q., Screening and Identification for Immunological Active Components from Andrographis Herba Using Macrophage Biospecific Extraction Coupled with UPLC/Q-TOF-MS. Molecules 2018, 23, (5).

(75) Nagar, A.; Gujral, V. K.; Gupta, S. R., A new flavone from Terminalia arjuna fruits. Phytochemistry 1979, 18, (7), 1245-0.

(76) Luo, Y.; Zeng, W.; Huang, K.; Li, D.; Chen, W.; Yu, X.; Ke, X., Discrimination of Citrus reticulata Blanco and Citrus reticulata 'Chachi' as well as the Citrus reticulata 'Chachi' within different 
storage years using ultra high performance liquid chromatography quadrupole/time-of-flight mass spectrometry based metabolomics approach. J. Pharmaceut. Biomed. 2019, 171, 218-231.

(77) Yang, C.; Fan, J.; Yang, J.; Gao, X., Flavonoids from Bauhinia championii Benth. Xibei Shifan Daxue Xuebao, Ziran Kexueban 2016, 52, (1), 75-78.

(78) Chen, C. C.; Chen, Y. P.; Hsu, H. Y.; Chen, Y. L., New flavones from Bauhinia championii Benth. Chem. Pharm. Bull. 1984, 32, (1), 166-9.

(79) Zhang, J. Y.; Li, N.; Che, Y. Y.; Zhang, Y.; Liang, S. X.; Zhao, M. B.; Jiang, Y.; Tu, P. F., Characterization of seventy polymethoxylated flavonoids (PMFs) in the leaves of Murraya paniculata by on-line high-performance liquid chromatography coupled to photodiode array detection and electrospray tandem mass spectrometry. J Pharm Biomed Anal 2011, 56, (5), 950-61.

(80) Li, P.; Zhang, Y.; Wang, Z.; Chen, H.; Li, X.; Jin, Y., A novel flavone from the leaves of Murraya paniculata. Chem. Nat. Compd. + 2018, 54, (6), 1061-1063.

(81) Dal Piaz, F.; Bader, A.; Malafronte, N.; D'Ambola, M.; Petrone, A. M.; Porta, A.; Ben Hadda, T.; De Tommasi, N.; Bisio, A.; Severino, L., Phytochemistry of compounds isolated from the leaf-surface extract of Psiadia punctulata (DC.) Vatke growing in Saudi Arabia. Phytochemistry (Elsevier) 2018, 155, 191-202.

(82) Wang, X.; Wei, R.; Lu, W.; Chen, J., Chemical composition of Callicarpa integerrima. Zhongcaoyao 1986, 17, (3), 108.

(83) Liu, J.; Ling, Y.; Wang, R.; Liu, G., Studies on flavonoids of Ardisia mamillata. Zhongcaoyao 2015, 46, (6), 808-811.

(84) Wei, G.; Hwang, L. S.; Tsai, C., Absolute bioavailability, pharmacokinetics and excretion of 5,7,3',4'-tetramethoxyflavone in rats. J. Funct. Foods 2014, 7, 136-141.

(85) Zengin, G.; Ferrante, C.; Gnapi, D. E.; Sinan, K. I.; Orlando, G.; Recinella, L.; Diuzheva, A.; Jeko, J.; Cziaky, Z.; Chiavaroli, A.; Leone, S.; Brunetti, L.; Picot-Allain, C.; Mahomoodally, M. F.; Angelini, P.; Covino, S.; Venanzoni, R.; Tirillini, B.; Menghini, L., Comprehensive approaches on the chemical constituents and pharmacological properties of flowers and leaves of American basil (Ocimum americanum L). Food Res. Int. 2019, 125, 108610.

(86) Ortega, A.; Maldonado, E.; Márquez, I.; Chiang, F., Coumarins and Flavones from Casimiroa pringlei. Heterocycles 2008, 75, (12), 3057-3064.

(87) Shin, H. J.; Nam, J.; Yoon, U. J.; Han, A.; Seo, E., Identification of three new flavonoids from 
the peels of Citrus unshiu. Helv. Chim. Acta 2012, 95, (2), 240-245.

(88) Encarnacion, R.; Ochoa, N.; Anthoni, U.; Christophersen, C.; Nielsen, P. H., Two new flavones from Calliandra californica. J. Nat. Prod. 1994, 57, (9), 1307-9.

(89) Zhao, X. J.; Xing, T. T.; Li, Y. F.; Jiao, B. N.; Jiang, D., Efficient analysis of phytochemical constituents in the peel of Chinese wild citrus Mangshanju (Citrus reticulata Blanco) by ultra high performance liquid chromatography-quadrupole time-of-flight-mass spectrometry. J. Sep. Sci. 2018, 41, (9), 1947-1959.

(90) Cao, C.; Liu, B.; Shen, W.; Wang, H.; Li, Y., [Research on antiproliferative effect of flavones isolated from Laggera pterodonta]. Zhongguo Zhong Yao Za Zhi 2010, 35, (16), 2171-4.

(91) Uckoo, R. M.; Jayaprakasha, G. K.; Vikram, A.; Patil, B. S., Polymethoxyflavones Isolated from the Peel of Miaray Mandarin (Citrus miaray) Have Biofilm Inhibitory Activity in Vibrio harveyi. J. Agr. Food Chem. 2015, 63, (32), 7180-7189.

(92) Ninomiya, K.; Matsumoto, T.; Chaipech, S.; Miyake, S.; Katsuyama, Y.; Tsuboyama, A.; Pongpiriyadacha, Y.; Hayakawa, T.; Muraoka, O.; Morikawa, T., Simultaneous quantitative analysis of 12 methoxyflavones with melanogenesis inhibitory activity from the rhizomes of Kaempferia parviflora. J Nat Med 2016, 70, (2), 179-89.

(93) Dominguez, X. A.; Meneses, A. Y., Friedelan-3,28-diol and a tetra-o-methylmorin from Mortonia palmeri. Phytochemistry 1974, 13, (7), 1292-1293.

(94) Sohrab, M. H.; Chowdhury, R.; Hasan, C. M.; Rashid, M. A., Chemotaxonomic significance of polyoxygenated flavonoids from the leaves of Micromelum minutum. Biochemical Systematics \& Ecology 2004, 32, (9), 829-831.

(95) Wang, Y.; Qian, J.; Cao, J.; Wang, D.; Liu, C.; Yang, R.; Li, X.; Sun, C., Antioxidant capacity, anticancer ability and flavonoids composition of 35 citrus (Citrus reticulata blanco) varieties. Molecules 2017, 22, (7), 1114/1-1114/20.

(96) Wang, Q. W.; Yu, D. H.; Lin, M. G.; Zhao, M.; Zhu, W. J.; Lu, Q.; Li, G. X.; Wang, C.; Yang, Y. F.; Qin, X. M.; Fang, C.; Chen, H. Z.; Yang, G. H., Antiangiogenic polyketides from Peperomia dindygulensis Miq. Molecules 2012, 17, (4), 4474-83.

(97) Tung, Y.; Chang, W.; Li, S.; Wu, J.; Badmeav, V.; Ho, C.; Pan, M., Citrus peel extracts attenuated obesity and modulated gut microbiota in mice with high-fat diet-induced obesity. Food Funct. 2018, 9, (6), 3363-3373. 
(98) Xing, T. T.; Zhao, X. J.; Zhang, Y. D.; Li, Y. F., Fast Separation and Sensitive Quantitation of Polymethoxylated Flavonoids in the Peels of Citrus Using UPLC-Q-TOF-MS. J. Agr. Food Chem. 2017, $65,(12), 2615-2627$.

(99) Wollenweber, E.; Dorr, M.; Rivera, D.; Roitman, J. N., Externally accumulated flavonoids in three Mediterranean Ononis species. Z Naturforsch C J Biosci 2003, 58, (11-12), 771-775.

(100) Medjroubi, K.; Benayache, F.; Benayache, S.; Akkal, S.; Kaabeche, M.; Tillequin, F.; Seguin, E., Eudesmanolide from Centaurea granata. Phytochemistry 1998, 49, (8), 2425-2427.

(101) Ayatollahi, A. M.; Ghanadian, M.; Att-ur-Rahman, R.; Mesaik, M. A.; Khalid, A. S.; Adeli, F., Methoxylated flavones from Salvia Mirzayanii Rech. f. and Esfand with immunosuppressive properties. Iran. J. Pharm. Res. 2015, 14, (3), 955-960.

(102) Belkacem, S.; Belbache, H.; Boubekri, C.; Mosset, P.; Rached-Mosbah, O.; Marchioni, E.; Benayache, S.; Benayache, F., Chemical constituents from Centaurea parviflora Desf. Research Journal of Pharmaceutical, Biological and Chemical Sciences 2014, 5, (3), 1275-1279, 5.

(103) Santi, M. D.; Bouzidi, C.; Gorod, N. S.; Puiatti, M.; Michel, S.; Grougnet, R.; Ortega, M. G., In vitro biological evaluation and molecular docking studies of natural and semisynthetic flavones from Gardenia oudiepe (Rubiaceae) as tyrosinase inhibitors. Bioorg. Chem. 2019, 82, 241-245.

(104) Alwahsh, M. A. A.; Khairuddean, M.; Chong, W. K., Chemical constituents and antioxidant activity of Teucrium barbeyanum Aschers. Rec. Nat. Prod. 2015, 9, (1), 159-163/1-159-163/5, 5 pp.

(105) Liu, Y.; Cheng, Y.; Liaw, C.; Chen, C.; Guh, J.; Hwang, T.; Tsai, J.; Wang, W.; Shen, Y., Bioactive Diterpenes from Callicarpa longissima. J. Nat. Prod. 2012, 75, (4), 689-693.

(106) Vidar, W. S.; Macabeo, A. P. G.; Knorn, M.; Kohls, P.; Aguinaldo, A. M., Polymethoxylated flavones from Micromelum compressum. Biochem. Syst. Ecol. 2013, 50, 48-50.

(107) K., S. M.; C., K. C.; T., P. C., 5-Hydroxy-7, 8, 2', 4'-tetramethoxyflavone from Limnophila rugosa. Phytochemistry 1989, 28, (6), 1778-1779.

(108) Lee, S.; Morita, H.; Tezuka, Y., Preferentially Cytotoxic Constituents of Andrographis paniculata and their Preferential Cytotoxicity against Human Pancreatic Cancer Cell Lines. Nat. Prod. Commun. 2015, 10, (7), 1934578X1501000704.

(109) Zhang, H.; Tian, G.; Zhao, C.; Han, Y.; DiMarco-Crook, C.; Lu, C.; Bao, Y.; Li, C.; Xiao, H.; Zheng, J., Characterization of polymethoxyflavone demethylation during drying processes of citrus peels. Food Funct. 2019, 10, (9), 5707-5717. 
(110) Toppo, E.; Darvin, S. S.; Esakkimuthu, S.; Stalin, A.; Balakrishna, K.; Sivasankaran, K.; Pandikumar, P.; Ignacimuthu, S.; Al-Dhabi, N. A., Antihyperlipidemic and hepatoprotective effects of Gardenin A in cellular and high fat diet fed rodent models. Chem Biol Interact 2017, 269, 9-17.

(111) Maurya, P.; Singh, S.; Gupta, M. M.; Luqman, S., Characterization of bioactive constituents from the gum resin of Gardenia lucida and its pharmacological potential. Biomed. Pharmacother. 2017, 85, 444-456.

(112) Kulkarni, M. M.; Rojatkar, S. R.; Nagasampagi, B. A., Four 6-hydroxyflavonols from Blumea malcomii. Phytochemistry 1987, 26, (7), 2079-2083.

(113) Hussain, J.; Begum, N.; Hussain, H.; Khan, F. U.; Rehman, N. U.; Al-Harrasi, A.; Ali, L., Ajuganane: a new phenolic compound from Ajuga bracteosa. Nat. Prod. Commun. 2012, 7, (5), 615-6.

(114) Happi, E. N.; Mpondo, T. N., Two Polymethoxylated Flavones from Distemonanthus benthamianus. J. Nat. Prod. 1994, 57, (2), 291-293.

(115) Iwase, Y.; Takemura, Y.; Ju-ichi, M.; Ito, C.; Furukawa, H.; Kawaii, S.; Yano, M.; Mou, X. Y.; Takayasu, J.; Tokuda, H.; Nishino, H., Inhibitory effect of flavonoids from citrus plants on Epstein-Barr virus activation and two-stage carcinogenesis of skin tumors. Cancer Lett. 2000, 154, (1), 101-5.

(116) Alarif, W. M.; Abdel-Lateff, A.; Al-Abd, A. M.; Basaif, S. A.; Badria, F. A.; Shams, M.; Ayyad, S. E., Selective cytotoxic effects on human breast carcinoma of new methoxylated flavonoids from Euryops arabicus grown in Saudi Arabia. Eur. J. Med. Chem. 2013, 66, 204-10.

(117) Chen, J. J.; Lin, R. W.; Duh, C. Y.; Huang, H. Y.; Chen, I. S., Flavones and Cytotoxic Constituents from the Stem Bark of Muntingia Calabura. J. Chin. Chem. Soc.-Taip. 2013, 51, (3), 665-670.

(118) Cai, X.; Yang, F.; Zhu, L.; Xia, Y.; Wu, Q.; Xue, H.; Lu, Y., Rosmarinic Acid, the Main Effective Constituent of Orthosiphon stamineus, Inhibits Intestinal Epithelial Apoptosis Via Regulation of the Nrf2 Pathway in Mice. Molecules 2019, 24, (17).

(119) Lin, L.; Chiou, C.; Cheng, J., 5-Deoxyflavones with Cytotoxic Activity from Mimosa diplotricha. J. Nat. Prod. 2011, 74, (9), 2001-2004.

(120) Al-Dabbas, M. M.; Al-Ismail, K.; Abu-Taleb, R.; Hashimoto, F.; Rabah, I. O.; Kitahara, K.; Fujita, K.; Suganuma, T., Chemistry and antiproliferative activities of 3-methoxyflavones isolated fromVarthemia iphionoides. Chem. Nat. Compd.+ 2011, 47, (1), 17-21.

(121) Leovigildo, Q.; Federico, G.; Guillermina, G.; Tirso, R., Flavonoids from Ageratum corymbosum. Phytochemistry 1992, 31, (8), 2859 - 2862. 
(122) Iinuma, M.; Matsuura, S.; Kurogochi, K.; Tanaka, T., Studies on the constituents of useful plants.

V. Multisubstituted flavones in the fruit peel of Citrus reticulata and their examination by gas-liquid chromatography. Chem. Pharm. Bull. 1980, 28, (3), 717-22.

(123) Kuo, W. L.; Liao, H. R.; Chen, J. J., Biflavans, Flavonoids, and a Dihydrochalcone from the Stem Wood of Muntingia calabura and Their Inhibitory Activities on Neutrophil Pro-Inflammatory Responses. Molecules 2014, 19, (12), 20521-20535.

(124) Carvalho, A. C.; Lira, J.; Pereira, T. M.; Silva, S. C.; Simote-Silva, S. Y.; Oliveira, F.; King-Diaz, B.; Lotina-Hennsen, B.; Veiga, T., Natural products from Pluchea sagittalis act as inhibitors of photosynthesis in vitro. Nat. Prod. Res. 2019, 33, (4), 557-562.

(125) Song, Y.; Desta, K. T.; Kim, G. S.; Lee, S. J.; Lee, W. S.; Kim, Y. H.; Jin, J. S.; Abd, E. A.; Shin, H. C.; Shim, J. H.; Shin, S. C., Polyphenolic profile and antioxidant effects of various parts of Artemisia annua $\quad$ L. Biomed. Chromatogr. 2016, 30, (4), 588-95.

(126) Wang, X. L.; Hu, M.; Wang, S.; Cheng, Y. X., Phenolic Compounds and Steroids fromRumex patientia. Chem. Nat. Compd.+ 2014, 50, (2), 311-313.

(127) Chang, R.; Wang, C.; Zeng, Q.; Guan, B.; Zhang, W.; Jin, H., Chemical constituents of the stems of Celastrus rugosus. Arch. Pharm. Res. 2013, 36, (11), 1291-301.

(128) Cao, C.; Liu, B.; Zeng, C.; Lu, Y.; Chen, S.; Yang, L.; Li, B.; Li, Y.; Li, Y., A polymethoxyflavone from Laggera pterodonta induces apoptosis in imatinib-resistant K562R cells via activation of the intrinsic apoptosis pathway. Cancer Cell Int. 2014, 14, (1), 137.

(129) Sato, A.; Tamura, H., High antiallergic activity of 5,6,4'-trihydroxy-7,8,3'-trimethoxyflavone and 5,6-dihydroxy-7,8,3',4'-tetramethoxyflavone from eau de cologne mint (Menthaxpiperita citrata). Fitoterapia 2015, 102, 74-83.

(130) LOYOLA, L. A.; NARANJO, J.; MORALES, G., 5,7-DIHYDROXY-3,8,3',4'TETRAMETHOXYFLAVONE FROM PARASTREPHIA-QUADRANGULARIS. Phytochemistry 1985, 24, (8), 1871-1872.

(131) Chen, J.; Montanari, A. M., Isolation and identification of new polymethoxyflavonoids from Dancy tangerine leaves. J. Agr. Food Chem. 1998, 46, (4), 1235-1238.

(132) Xu, L.; Xu, J.; Zhong, K.; Shang, Z.; Wang, F.; Wang, R.; Zhang, L.; Zhang, J.; Liu, B., Analysis of non-volatile chemical constituents of menthae haplocalycis herba by ultra-high performance liquid chromatography-high resolution mass spectrometry. Molecules 2017, 22, (10), 1756/1-1756/17. 
(133) El Marsni, Z.; Torres, A.; Varela, R. M.; Molinillo, J. M. G.; Casas, L.; Mantell, C.; Martinez De La Ossa, E. J.; Macias, F. A., Isolation of bioactive compounds from sunflower leaves (Helianthus annuus L.) extracted with supercritical carbon dioxide. J. Agr. Food Chem. 2015, 63, (28), 6410-6421.

(134) Liu, S.; Liao, Z.; Liu, C.; Ji, L.; Sun, H., Two new sesquiterpenes from Artemisia sieversiana. Fitoterapia 2014, 97, 43-49.

(135) Kozhiparambil, K. P.; Ayyapath, S.; Ganesan, S.; Joseph, D. C., 5,7-dihydroxy-6, 2', 4', 5'tetramethoxyflavone from the leaves of Chukrasia Tabularis. Phytochemistry 1977, 16, (3), 398-399.

(136) Fu, M. Q.; Xiao, G. S.; Wu, J. J.; Chen, Y. L.; Zou, B.; An, K. J.; Xu, Y. J., Chemical Constituents from Pericarpium Citri Reticulatae. Chinese Herbal Medicines 2017, 9, (1), 86-91.

(137) Mai, L. H.; Chabot, G. G.; Grellier, P.; Quentin, L.; Dumontet, V.; Poulain, C.; Espindola, L. S.; Michel, S.; Vo, H. T.; Deguin, B.; Grougnet, R., Antivascular and anti-parasite activities of natural and hemisynthetic flavonoids from New Caledonian Gardenia species (Rubiaceae). Eur. J. Med. Chem. 2015, 93, 93-100.

(138) Cimanga, K.; Ying, L.; De Bruyne, T.; Apers, S.; Cos, P.; Hermans, N.; Bakana, P.; Tona, L.; Kambu, K.; Kalenda, D. T.; Pieters, L.; Vanden Berghe, D.; Vlietinck, A. J., Radical scavenging and xanthine oxidase inhibitory activity of phenolic compounds from Bridelia ferruginea stem bark. J. Pharm. Pharmacol. 2001, 53, (5), 757-761.

(139) Tuchinda, P.; Saiai, A.; Pohmakotr, M.; Yoosook, C.; Kasisit, J.; Napaswat, C.; Santisuk, T.; Reutrakul, V., Anti-HIV-1 cycloartanes from leaves and twigs of Gardenia thailandica. Planta Med. 2004, $70,(4), 366-70$.

(140) Juma, B. F.; Yenese, A.; Midiwo, J. O.; Waterman, P. G., Flavones and phenylpropenoids in the surface exudate of Psiadia punctulata. Phytochemistry 2001, 57, (4), 571-4.

(141) Deng, Y. R.; Song, A. X.; Wang, H. Q., Chemical components of Seriphidium santolium Poijak. J. Chin. Chem. Soc.-Taip. 2004, 51, (3), 629-636.

(142) Wollenweber, E.; Mann, K.; Arriaga-Giner, F. J.; Roitman, J. N.; West, J. G., Exudate flavonoids from two Australian Asteraceae, Bracteantha viscosa and Cassinia quinquefaria. Phytochemistry 1993, $33,(4), 871-3$.

(143) Long, H. L.; Xu, G. Y.; Deng, A. J.; Li, Z. H.; Ma, L.; Lu, Y.; Zhang, Z. H.; Wu, F.; Qin, H. L., Two new flavonoids from the roots of Scutellaria baicalensis. J. Asian Nat. Prod. Res. 2015, 17, (7), 75660. 
(144) Kongkum, N.; Tuchinda, P.; Pohmakotr, M.; Reutrakul, V.; Piyachaturawat, P.; Jariyawat, S.;

Suksen, K.; Yoosook, C.; Kasisit, J.; Napaswad, C., DNA topoisomerase IIalpha inhibitory and antiHIV-1 flavones from leaves and twigs of Gardenia carinata. Fitoterapia 2012, 83, (2), 368-72.

(145) Monteillier, A.; Cretton, S.; Ciclet, O.; Marcourt, L.; Ebrahimi, S. N.; Christen, P.; Cuendet, M., Cancer chemopreventive activity of compounds isolated from Waltheria indica. J. Ethnopharmacol. 2017, 203, 214-225.

(146) Nhoek, P.; Chae, H. S.; Masagalli, J. N.; Mailar, K.; Pel, P.; Kim, Y. M.; Choi, W. J.; Chin, Y. W., Discovery of Flavonoids from Scutellaria baicalensis with Inhibitory Activity Against PCSK 9 Expression: Isolation, Synthesis and Their Biological Evaluation. Molecules 2018, 23, (2).

(147) Kuo, P. C.; Liao, Y. R.; Hung, H. Y.; Chuang, C. W.; Hwang, T. L.; Huang, S. C.; Shiao, Y. J.; Kuo, D. H.; Wu, T. S., Anti-Inflammatory and Neuroprotective Constituents from the Peels of Citrus grandis. Molecules 2017, 22, (6).

(148) Ifeanacho, M. O.; Ogunwa, S. C.; Amadi, P. U., Phytochemical Composition of Vitex doniana. Analytical Chemistry Letters 2019, 9, (6), 863-875.

(149) Costa, R.; Ragusa, S.; Russo, M.; Certo, G.; Franchina, F. A.; Zanotto, A.; Grasso, E.; Mondello, L.; Germano, M. P., Phytochemical screening of Artemisia arborescens L. by means of advanced chromatographic techniques for identification of health-promoting compounds. J Pharm Biomed Anal 2016, 117, 499-509.

(150) Nianbai, F.; Mark, L.; Tom, J. M., Fifty-one flavonoids from Gutierrezia microcephala. Phytochemistry 1986, 25, (4), 927-934.

(151) Kinoshita, T.; Firman, K., Highly oxygenated flavonoids from Murraya paniculata. Phytochemistry 1996, 42, (4), 1207-1210.

(152) Abdallah, H. M.; Zakaria, E. M.; El-Halawany, A. M.; Mohamed, G. A.; Safo, M. K.; El-Bassossy, H. M., Psiadia punctulata major flavonoids alleviate exaggerated vasoconstriction produced by advanced glycation end products. PLoS One 2019, 14, (9), e0222101.

(153) Kontogianni, V. G.; Primikyri, A.; Sakka, M.; Gerothanassis, I. P., Simultaneous determination of artemisinin and its analogs and flavonoids in Artemisia annua crude extracts with the use of NMR spectroscopy. Magn. Reson. Chem. 2020, 58, (3), 232-244.

(154) Fang, S. T.; Kong, N. N.; Yan, B. F.; Yang, C. Y.; Wang, J. H.; Liu, S. J.; Jin, H. Z.; Xia, C. H., Chemical constituents and their bioactivities from the fruits of Vitex negundo var. cannabifolia. Nat. 
Prod. Res. 2016, 30, (24), 2856-2860.

(155) Lee, H. J.; Sim, M. O.; Woo, K. W.; Jeong, D.; Jung, H. K.; An, B.; Cho, H. W., Antioxidant and Antimelanogenic Activities of Compounds Isolated from the Aerial Parts of Achillea alpina L. Chem. Biodivers. 2019, 16, (7), n/a.

(156) Promchai, T.; Jaidee, A.; Cheenpracha, S.; Trisuwan, K.; Rattanajak, R.; Kamchonwongpaisan, S.; Laphookhieo, S.; Pyne, S. G.; Ritthiwigrom, T., Antimalarial Oxoprotoberberine Alkaloids from the Leaves of Miliusa cuneata. J. Nat. Prod. 2016, 79, (4), 978-983.

(157) Cheng, X.; Jie, R.; Wang, C.; Guan, B.; Jin, H.; Zhang, W., Chemical Constituents from Inula wissmanniana. Chem. Nat. Compd.+ 2013, 49, (5), 815-818.

(158) Yu, S.; Fang, N.; Mabry, T. J., Flavonoids from Gymnosperma glutinosum. Phytochemistry 1988, 27, (1), 171-177.

(159) Abbaszadeh, H.; Ebrahimi, S. A.; Akhavan, M. M., Antiangiogenic activity of xanthomicrol and calycopterin, two polymethoxylated hydroxyflavones in both in vitro and ex vivo models. Phytother. Res. 2014, 28, (11), 1661-70.

(160) WAGNER, H.; MAURER, G.; HORHAMMER, L.; FARKAS, L., STRUCTURE OF A FLAVONE FROM CITRUS-RETICULATA BLANCO - SYNTHESIS OF 5.4'-DIHYDROXY-6.7.8.3' TETRAMETHOXYFLAVONE. CHEMISCHE BERICHTE-RECUEIL 1971, 104, (11), 3357-+.

(161) Brentan, S. D.; Aschenbrenner, A. K.; Lopes, N. P.; Spring, O., Direct Analyses of Secondary Metabolites by Mass Spectrometry Imaging (MSI) from Sunflower (Helianthus annuus L.) Trichomes. Molecules 2017, 22, (5).

(162) SOUZA, M. F.; CUNHA, G.; FONTENELE, J. B.; VIANA, G.; RAO, V.; SILVEIRA, E. R., ANTITHROMBOTIC ACTIVITY OF TERNATIN, A TETRAMETHOXY FLAVONE FROM EGLETES VISCOSA LESS. Phytother. Res. 1994, 8, (8), 478-481.

(163) Kennedy, M. L.; Cortes, F.; Pinero, J. E.; Castanys, S.; Lopez-Arencibia, A.; Gamarro, F.; Bazzocchi, I. L.; Jimenez, I. A., Leishmanicidal and reversal multidrug resistance constituents from Aeonium lindleyi. Planta Med. 2011, 77, (1), 77-80.

(164) Kumari, G. N. K.; Rao, L. J. M.; Rao, N. S. P., Myricetin methyl ethers from Solanum pubescens. Phytochemistry (Elsevier) 1984, 23, (11), 2701-2.

(165) JAY, M.; FAVREBONVIN, J.; WOLLENWEBER， E., 4',5-DIHYDROXY-2',3,7,8TETRAMETHOXYFLAVONE AND 5-HYDROXY-2',3,4',7,8-PENTAMETHOXYFLAVONE， 2 
NEW NATURAL COMPOUNDS ISOLATED FROM NOTHOLAENA-AFFINIS (PTERIDOPHYTAE). CANADIAN JOURNAL OF CHEMISTRY-REVUE CANADIENNE DE CHIMIE 1979, 57, (14), 1901-1902.

(166) Nikonov, G. K.; Safronich, L. N.; Pimenov, M. G., Chrysosplin a new flavonoid from Chrysosplenium pseudo-faurieri. Khimiya Prirodnykh Soedinenii 1970, 6, (2), 268-9.

(167) Santi, M. D.; Paulino, Z. M.; Vera, B.; Bouzidi, C.; Dumontet, V.; Abin-Carriquiry, A.; Grougnet, R.; Ortega, M. G., Xanthine oxidase inhibitory activity of natural and hemisynthetic flavonoids from Gardenia oudiepe (Rubiaceae) in vitro and molecular docking studies. Eur. J. Med. Chem. 2018, 143, $577-582$.

(168) Arisawa, M.; Hayashi, T.; Shimizu, M.; Morita, N.; Bai, H.; Kuze, S.; Ito, Y., Isolation and cytotoxicity of two new flavonoids from Chrysosplenium grayanum and related flavonols. J. Nat. Prod. 1991, 54, (3), 898-901.

(169) Malan, E.; Swinny, E.; Ferreira, D.; Hall, A. J., 5,5'-Dihydroxy-3,7,2',4'-tetramethoxyflavone from Chlorophora excelsa. Phytochemistry 1990, 29, (8), 2741.

(170) Gunatilaka, A. A. L.; Sarath, R. S.; Subramaniam, S.; H., T. B. S., Flavonoids of Gardenia cramerii and G. fosbergii bud exudates. 1982, 21, (3), 805-806.

(171) Wang, H.; Hui, K. M.; Xu, S.; Chen, Y.; Wong, J. T.; Xue, H., Two flavones from Scutellaria baicalensis Georgi and their binding affinities to the benzodiazepine site of the GABAA receptor complex. Pharmazie 2002, 57, (12), 857-8.

(172) Balah, M. A. A., Allelopathic potential of Jasonia montana (wild plant) to control weeds of family Convolvulaceae. Allelopathy J. 2012, 29, (1), 93-106.

(173) Huang, Y.; Ding, Z. H.; Liu, J. K., A new highly oxygenated flavone from Veronia saligna. $Z$ Naturforsch C J Biosci 2003, 58, (5-6), 347-50.

(174) Lichius, J. J.; Thoison, O.; Montagnac, A.; Pais, M.; Gueritte-Voegelein, F.; Sevenet, T.; Cosson, J. P.; Hadi, A. H., Antimitotic and cytotoxic flavonols from Zieridium pseudobtusifolium and Acronychia porteri. J. Nat. Prod. 1994, 57, (7), 1012-6.

(175) Kinoshita, T., A new flavonol from Murraya paniculata var. omphalocarpa: 13C-NMR as a useful tool for structure elucidation of polyoxyflavones. Heterocycles 1999, 50, (1), 269-276.

(176) Habtemariam, S.; Gray, A. I.; Waterman, P. G., Flavonoids from three Ethiopian species of Premna. Zeitschrift fuer Naturforschung, B: Chemical Sciences 1992, 47, (1), 144-7. 
(177) Nianbai, F.; Mark, L.; T., J. M.; Iinuma, M., Six 2'-hydroxyflavonols from Gutierrezia microcephala. Phytochemistry 1985, 24, (12), 3029-3034.

(178) Akimanya, A.; Midiwo, J. O.; Matasyoh, J.; Okanga, F.; Masila, V. M.; Walker, L.; Tekwani, B. L.; Muhammad, I.; Omosa, L. K., Two polymethoxylated flavonoids with antioxidant activities and a rearranged clerodane diterpenoid from the leaf exudates of Microglossa pyrifolia. Phytochem. Lett. 2015, $11,183-187$.

(179) L., Q.; J., S. C.; F., G. G.; I., E. S.; T., R., Highly oxygenated flavonoids from Ageratum corymbosum. Phytochemistry 1980, 19, (11), 2439-2442.

(180) MUSCHIETTI, L.; MARTINO, V.; FERRARO, G.; COUSSIO, J., 5,7,5'-TRIHYDROXY3,6,2',4'-TETRAMETHOXYFLAVONE FROM EUPATORIUM-BUNIIFOLIUM. Phytochemistry 1994, 36, (4), 1085-1086.

(181) Collins, F. W.; De Luca, V.; Ibrahim, R. K.; Voirin, B.; Jay, M., Polymethylated flavonols of Chrysosplenium americanum. I. Identification and enzymic syntheses. Zeitschrift fuer Naturforschung, C: Journal of Biosciences 1981, 36C, (9-10), 730-6.

(182) Jay, M.; Favre-Bonvin, J.; Voirin, B.; Viricel, M. R.; Wollenweber, E., A new natural flavone with a tetrasubstituted B-ring from the fern Notholaena aschenborniana. Phytochemistry (Elsevier) 1981, $20,(9), 2307-8$.

(183) Seo, S.; Lee, Y.; Moon, H., Acetyl-cholinesterase Inhibitory Activity of Methoxyflavones Isolated from Kaempferia parviflora. Nat. Prod. Commun. 2017, 12, (1), 1934578X1701200107.

(184) Sichaem, J.; Tip-Pyang, S.; Siripong, P., Chemical Constituents from the Root Bark of Calophyllum inophyllum. Nat. Prod. Commun. 2018, 13, (6), $1934578 X 1801300618$.

(185) Bellete, B. S.; Ramin, L. Z.; Porto, D.; Ribeiro, A. I.; Forim, M. R.; Zuin, V. G.; Fernandes, J. B.; Silva, M. F. G. F., An environmentally friendly procedure to obtain flavonoids from Brazilian citrus waste. J. Brazil. Chem. Soc. 2018, 29, (5), 1123-1129.

(186) Imran, M.; Irfan, A.; Ibrahim, M.; Assiri, M. A.; Khalid, N.; Ullah, S.; Al-Sehemi, A. G., Carbonic anhydrase and cholinesterase inhibitory activities of isolated flavonoids from Oxalis corniculata L. and their first-principles investigations. Ind. Crop. Prod. 2020, 148, 112285.

(187) Pan, W.; Wei, L.; Wei, L.; Wu, Y., Chemical constituents of Tupistra chinensis rhizomes. Chem. Pharm. Bull. 2006, 54, (7), 954-958.

(188) Guo, C.; Shan, Y.; Yang, Z.; Zhang, L.; Ling, W.; Liang, Y.; Ouyang, Z.; Zhong, B.; Zhang, J., 
Chemical composition, antioxidant, antibacterial, and tyrosinase inhibition activity of extracts from Newhall navel orange (Citrus sinensis Osbeck cv. Newhall) peel. J. Sci. Food Agr. 2020, 100, (6), 26642674.

(189) Lu, Y.; Zhu, S.; He, Y.; Mo, C.; Wu, C.; Zhang, R.; Zheng, X.; Tang, Q., Systematic characterization of flavonoids from Siraitia grosvenorii leaf extract using an integrated strategy of highspeed counter-current chromatography combined with ultra high performance liquid chromatography and electrospray ionization quadrupole time-of-flight mass spectrometry. J. Sep. Sci. 2020, 43, (5), 852864.

(190) Mostafa, N. M.; Abd, E. E.; Hegazy, H. G.; Eldahshan, O. A., New Methoxyflavone from Casimiroa sapota and the Biological Activities of Its Leaves Extract against Lead Acetate Induced Hepatotoxicity in Rats. Chem. Biodivers. 2018, 15, (4), e1700528.

(191) Heinrich, M.; Heneka, B.; Ankli, A.; Rimpler, H.; Sticher, O.; Kostiza, T., Spasmolytic and antidiarrhoeal properties of the Yucatec Mayan medicinal plant Casimiroa tetrameria. J. Pharm. Pharmacol. 2005, 57, (9), 1081-1085.

(192) Dominguez, X. A.; Villegas, D.; Rodriguez, V. M.; Zamora, G., Structure of cerrosillin B $\left(5,6,3^{\prime}, 4^{\prime}, 5^{\prime}\right.$-pentamethoxyflavone) isolated from the leaves of chapote amarillo (Sargentia greggii). Revista Latinoamericana de Quimica 1976, 7, (1), 45-6.

(193) Itoh, T.; Ohguchi, K.; Iinuma, M.; Nozawa, Y.; Akao, Y., Inhibitory effects of polymethoxy flavones isolated from Citrus reticulate on degranulation in rat basophilic leukemia RBL-2H3: enhanced inhibition by their combination. Bioorg Med Chem 2008, 16, (16), 7592-8.

(194) Tomazela, D. M.; Pupo, M. T.; Passador, E. A.; Da, S. M.; Vieira, P. C.; Fernandes, J. B.; Fo, E. R.; Oliva, G.; Pirani, J. R., Pyrano chalcones and a flavone from Neoraputia magnifica and their Trypanosoma cruzi glycosomal glyceraldehyde-3-phosphate dehydrogenase-inhibitory activities. Phytochemistry 2000, 55, (6), 643-51.

(195) Liang, Q.; Yang, S. X.; Kuang, Y.; Liu, L., Chemical constituents from stems of Pleioblastus amarus. Chinese Traditional \& Herbal Drugs 2015, 46, (8), 1125-1128.

(196) Hari Kishore, P.; Vijaya Bhaskar Reddy, M.; Kesava Reddy, M.; Gunasekar, D.; Caux, C.; Bodo, B., Flavonoids from Andrographis lineata. Phytochemistry (Elsevier) 2003, 63, (4), 457-461.

(197) Deepa, V. S.; Kumar, P. S.; Vadivukkarasi, B.; Tilton, F., Molecular docking studies on selected phytocompounds from different Andrographis sp. against PPAR- $\gamma$ and C/EBP- $\alpha$ receptors for type- 
2-diabetes. Asian Journal of Pharmaceutical and Clinical Research 2013, 6, (Suppl. 2), 196-199.

(198) Kinoshita, T.; Firman, K., Myricetin 5,7,3',4',5'-pentamethyl ether and other methylated flavonoids from Murraya paniculata. Phytochemistry 1997, 45, (1), 179-181.

(199) Li, S.; Lo, C. Y.; Ho, C. T., Hydroxylated polymethoxyflavones and methylated flavonoids in sweet orange (Citrus sinensis) peel. J Agric Food Chem 2006, 54, (12), 4176-85.

(200) Ezzatzadeh, E.; Farjam, M. H.; Rustaiyan, A., Comparative evaluation of antioxidant and antimicrobial activity of crude extract and secondary metabolites isolated from Artemisia kulbadica. Asian Pacific Journal of Tropical Disease 2012, 2, (Suppl. 1), S431-S434.

(201) Rustaiyan, A.; Ezzatzadeh, E., Sesquiterpene lactones and penta methoxylated flavone from Artemisia kulbadica. Asian Journal of Chemistry 2011, 23, (4), 1774-1776.

(202) Tambewagh, U. U.; Javir, G. R.; Joshi, K. S.; Rojatkar, S. R., Two new polyoxygenated flavonoids from Blumea eriantha DC, methanol extract and their anti-proliferative activity. Nat. Prod. Res. 2019, Ahead of Print.

(203) Li, S.; Wang, Y.; Wang, Z.; Xiao, H.; Lo, C. Y.; Rawson, N.; Ho, C. T., Quantitative analysis of hydroxylated polymethoxyflavones by high-performance liquid chromatography. Biomed. Chromatogr. 2010, 24, (8), 838-45.

(204) Elkin, Y. N.; Kulesh, N. I.; Stepanova, A. Y.; Solovieva, A. I.; Kargin, V. M.; Manyakhin, A. Y., Methylated flavones of the hairy root culture Scutellaria baicalensis. J. Plant Physiol. 2018, 231, $277-$ 280.

(205) AHMAD, V. U.; ISMAIL, N., 5-HYDROXY-3,6,7,2',5'-PENTAMETHOXYFLAVONE FROM INULA-GRANTIOIDES. Phytochemistry 1991, 30, (3), 1040-1041.

(206) Luo, D.; Zhang, Y.; Huang, J.; Chen, L.; He, L.; Kuang, G.; Qin, J.; Li, Q.; Wang, G.; Li, Y., One New Sesquiterpene from the Leaves of Rhodomyrtus tomentosa. Chem. Lett. 2018, 48.

(207) Auzi, A. A.; Gray, A. I.; Hartley, T. G.; Waigh, R. D.; Waterman, P. G., A xanthine alkaloid from the leaves of Bosistoa floydii. Phytochemistry 1994, 36, (2), 535-6.

(208) Sichaem, J.; Nguyen, H.; Nguyen, V.; Mac, D.; Mai, D.; Nguyen, H.; Tran, T.; Pham, N.; Nguyen, H.; Niamnont, N.; Duong, T., A new labdane-type diterpenoid from the leaves of Vitex negundo L. Nat. Prod. Res. 2019, Ahead of Print.

(209) Sharaf, M.; Abu-Gabal, N. S.; El-Ansari, M. A., Exudate flavonoids from Ficus altissima. Biochem. Syst. Ecol. 2000, 28, (3), 291-293. 
(210) Malik, A., Capspinosin, a New Flavonoid from Capparis spinosa. Journal Chemical Society of Pakistan 2013, 35, (3).

(211) Sawadogo, W. R.; Cerella, C.; Al-Mourabit, A.; Moriou, C.; Teiten, M. H.; Guissou, I. P.; Dicato, M.; Diederich, M., Cytotoxic, Antiproliferative and Pro-Apoptotic Effects of 5-Hydroxyl-6,7,3',4',5'Pentamethoxyflavone Isolated from Lantana ukambensis. Nutrients 2015, 7, (12), 10388-97.

(212) Silva, F. L.; Moreno, P. R. H.; Braz-Filho, R.; Tavares, J. F.; Barbosa-Filho, J. M., Chemical constituents of Cardiospermum corindum L. and their distribution in Sapindaceae. Biochemical Systematics \& Ecology 2014, 57, 137-140.

(213) Li, S.; Lo, C.; Dushenkov, S.; Ho, C., Polymethoxyflavones: chemistry, biological activity, and occurrence in orange peel. ACS Symposium Series 2008, 987, (Dietary Supplements), 191-210.

(214) Chen, J.; Montanari, A. M.; Widmer, W. W., Two New Polymethoxylated Flavones, a Class of Compounds with Potential Anticancer Activity, Isolated from Cold Pressed Dancy Tangerine Peel Oil Solids. J. Agr. Food Chem. 1997, 45, (2), 364-368.

(215) Mimaki, Y.; Sashida, Y.; Furuya, S.; Sakagami, H., Polymethoxylated flavonoids from the peels of Citrus depressa. Natural Medicines 2000, 54, (6), 351.

(216) CL, Z.; SX, F.; Q, W.; P, W.; J, X.; T., C., Flavonoids and Phenolic Compounds fromSmilax scobinicaulis. Chem. Nat. Compd.+ 2014, 50, (2), 254-257.

(217) Lin, Z.; Wang, H.; Xu, Y.; Dong, J.; Hashi, Y.; Chen, S., Identification of antioxidants in Fructus aurantii and its quality evaluation using a new on-line combination of analytical techniques. Food Chem. 2012, 134, (2), 1181-1191.

(218) Johann, S.; Smania, A. J.; Pizzolatti, M. G.; Schripsema, J.; Braz-Filho, R.; Branco, A., Complete $1 \mathrm{H}$ and 13C NMR assignments and anti fungal activity of two 8-hydroxy flavonoids in mixture. An. Acad. Bras. Cienc. 2007, 79, (2), 215-22.

(219) Ashok, V. V.; Newand, B. M., Polyoxygenated flavones from Ageratum conyzoides. Phytochemistry 1986, 25, (11), 2625-2627.

(220) Faqueti, L. G.; Sandjo, L. P.; Biavatti, M. W., Simultaneous identification and quantification of polymethoxyflavones, coumarin and phenolic acids in Ageratum conyzoides by UPLC-ESI-QToF-MS and UPLC-PDA. J. Pharmaceut. Biomed. 2017, 145, 621-628.

(221) Glabasnia, A.; Dunkel, A.; Frank, O.; Hofmann, T., Decoding the Nonvolatile Sensometabolome of Orange Juice (Citrus sinensis). J Agric Food Chem 2018, 66, (10), 2354-2369. 
(222) Qi, S.; Guo, J.; Wu, D.; Luo, X., Study on the chemical constituents of Ostodes paniculata. Tianran Chanwu Yanjiu Yu Kaifa 2004, 16, (3), 210-212.

(223) Simonsen, H. T.; Larsen, M. D.; Nielsen, M. W.; Adsersen, A.; Olsen, C. E.; Strasberg, D.; Smitt, U. W.; Jaroszewski, J. W., Methylenedioxy- and methoxyflavones from Melicope coodeana syn. Euodia simplex. Phytochemistry 2002, 60, (8), 817-20.

(224) Quijano, L.; Calderon, J. S.; Garibay, F. G.; Escobar, E.; Rios, T., Flavonoids from Ageratum species. Part 5. Further polysubstituted flavones from Ageratum houstonianum. Phytochemistry 1987, $26,(7), 2075-8$.

(225) Phromnoi, K.; Prasad, S.; Gupta, S. C.; Kannappan, R.; Reuter, S.; Limtrakul, P.; Aggarwal, B. B., Dihydroxypentamethoxyflavone down-regulates constitutive and inducible signal transducers and activators of transcription-3 through the induction of tyrosine phosphatase SHP-1. Mol. Pharmacol. 2011, $80,(5), 889-99$.

(226) Afifi, M. S., Phytochemical and biological investigation of Cleome brachycarpa Vahl. Growing in Egypt. International Journal of Pharmaceutical Sciences and Research 2014, 5, (9), 3726-3732.

(227) Reichelt, K. V.; Hoffmann-Lücke, P.; Hartmann, B.; Weber, B.; Ley, J. P.; Krammer, G. E.; Swanepoel, K. M.; Engel, K. H., Phytochemical characterization of South African bush tea (Athrixia phylicoides DC.). S. Afr. J. Bot. 2012, 83, (Complete), 1-8.

(228) Filho, R. B.; Gottlieb, O. R., The flavones of Apuleia leiocarpa. Phytochemistry 1971, 10, (10), $2433-2450$.

(229) Lerotholi, L. J.; Chaudhary, S. K.; Chen, W.; Veale, C.; Combrinck, S.; Viljoen, A. M., Identification, Isolation and Determination of Biomarkers for Quality Control of Bush Tea (Athrixia phyllicoides). Planta Med. 2018, 84, (12-13), 902-912.

(230) Oufedjikh, H.; Mahrouz, M.; Amiot, M. J.; Lacroix, M., Effect of $\gamma$-Irradiation on Phenolic Compounds and Phenylalanine Ammonia-Lyase Activity during Storage in Relation to Peel Injury from Peel of Citrus clementina Hort. Ex. Tanaka. J. Agr. Food Chem. 2000, 48, (2), 559-565.

(231) Al-Dabbas, M. M.; Kitahara, K.; Suganuma, T.; Hashimoto, F.; Tadera, K., Antioxidant and a -amylase inhibitory compounds from aerial parts of Varthemia iphionoides Boiss. Bioscience, Biotechnology, and Biochemistry 2006, 70, (9), 2178-2184.

(232) Zhong, W.; Luo, Y.; Li, J.; Wu, Y.; Gao, Y.; Luo, H.; Yang, Y.; Jiang, L., Polymethoxylated flavonoids from Citrus reticulata Blanco. Biochem. Syst. Ecol. 2016, 68, 11-14. 
(233) Matsuda, H.; Morikawa, T.; Yoshikawa, M., Antidiabetogenic constituents from several natural medicines. Pure \& Applied Chemistry 2002, 74, (7), 1301---1308.

(234) Sukari, M. A.; Riyanto, S.; Ali, A. M.; Yusof, U. K.; Haron, M. J.; Ahmad, F. B. H., Isolation of flavonoids from Murraya paniculata L. Oriental Journal of Chemistry 2001, 17, (1), 27-30.

(235) Oyama, M.; Nakashima, K.; Kamiya, T.; Haba, M.; Ito, T.; Murata, H.; Tanaka, T.; Adachi, T.; Iinuma, M.; Kinoshita, T., Flavonoids isolated from the leaves of Melicope triphylla and their extracellular-superoxide dismutase-inducing activity. Phytochem. Lett. 2013, 6, (2), 215-218.

(236) Butler, M. S.; Healy, P. C.; Forster, P. I.; Guymer, G. P.; Quinn, R. J., 5,6,7,3',4',5'Hexamethoxyflavone from the Australian plant Eremophila debilis (Myoporaceae). Fitoterapia 2018, $126,90-92$.

(237) Nagai, H.; Tanaka, T.; Goto, T.; Kusudo, T.; Takahashi, N.; Kawada, T., Phenolic compounds from leaves of Casimiroa edulis showed adipogenesis activity. Biosci Biotechnol Biochem 2014, 78, (2), 296-300

(238) Wu, S.; Li, J.; Wang, Q.; Cao, J.; Yu, H.; Cao, H.; Xiao, J., Chemical composition, antioxidant and anti-tyrosinase activities of fractions from Stenoloma chusanum. Ind. Crop. Prod. 2017, 107, 539545.

(239) Ferracin, R. J.; Da Silva, M. F. D. G.; Fernandes, J. B.; Vieira, P. C., Flavonoids from the fruits of Murraya paniculata. Phytochemistry 1997, 47, (3), 393-396.

(240) Chkhikvishvili, I. D.; Kurkin, V. A.; Pervykh, L. N., Flavonoids of fruit peel of Citrus unshiu. Khimiya Prirodnykh Soedinenii 1994,, (6), 821-2.

(241) de Beer, D.; Joubert, E.; Malherbe, C. J.; Jacobus, B. D., Use of countercurrent chromatography during isolation of 6-hydroxyluteolin-7-O-beta-glucoside, a major antioxidant of Athrixia phylicoides. J. Chromatogr. A 2011, 1218, (36), 6179-86.

(242) DESILVA, L. B.; DESILVA, U.; MAHENDRAN, M.; JENNINGS, R. C., 4'-HYDROXY3,5,6,7,3',5'-HEXAMETHOXYFLAVONE FROM MURRAYA-PANICULATA. Phytochemistry 1980, $19,(12), 2794-2794$.

(243) Rane Zab, A. K. P.; Bhaskar, A., Determination of bioactive components from the ethanolic peel extract of Citrus reticulata by gas chromatography - mass spectrometry. International Journal of Drug Development \& Research 2012, 4, (4), 166-174.

(244) Kostadinovic, S.; Jovanov, D.; Mirhosseini, H., Comparative investigation of cold pressed 
essential oils from peel of different mandarin varieties. IIOAB Journal 2012, 3, (4), 7-13.

(245) Ohtsuki, T.; Koyano, T.; Kowithayakorn, T.; Yamaguchi, N.; Ishibashi, M., Isolation of austroinulin possessing cell cycle inhibition activity from Blumea glomerata and revision of its absolute configuration. Planta Med. 2004, 70, (12), 1170-1173.

(246) Shajib, M. S.; Rashid, R. B.; Ming, L. C.; Islam, S.; Sarker, M.; Nahar, L.; Sarker, S. D.; Datta, B. K.; Rashid, M. A., Polymethoxyflavones from Nicotiana plumbaginifolia (Solanaceae) Exert Antinociceptive and Neuropharmacological Effects in Mice. Front Pharmacol 2018, 9, 85.

(247) PATWARDHAN, S. A.; GUPTA, A. S., AN OCTAMETHOXYFLAVONE FROM POGOSTEMON-PURPURASCENS. Phytochemistry 1981, 20, (6), 1458-1459.

(248) Chung, L. Y.; Yap, K. F.; Goh, S. H.; Mustafa, M. R.; Imiyabir, Z., Muscarinic receptor binding activity of polyoxygenated flavones from Melicope subunifoliolata. Phytochemistry 2008, 69, (7), 154854.

(249) Trieu, Q. H.; Mai, H. D. T.; Litaudon, M.; Thi, D. P.; Tran, T. A.; Nguyen, V. H.; Chau, V. M.; Pham, V. C., Two new linear acetogenins from the fruits of Goniothalamus gracilipes. Nat. Prod. Res. 2018, 32, (3), 287-293.

(250) Facundo, V. A.; Da Silveira, A. S. P.; Morais, S. M., Constituents of Piper alatabaccum Trel \& Yuncker (Piperaceae). Biochem. Syst. Ecol. 2005, 33, (7), 753-756.

(251) Chen, C.; Huang, Y.; Ou, J., Constituents of the stem of Bauhinia championii. Chinese Pharmaceutical Journal (Taipei, Taiwan) 1994, 46, (6), 485-9.

(252) Li, H. J.; Zhou, D. L.; Xu, T. J.; Lam, C. K.; Lan, W. J., 5,6,7,5'-Tetramethoxy-3',4'methylenedioxyflavone monohydrate. 2012, 68, (Pt 5), o1390-o1390.

(253) Le-Van, N.; Pham, T. V. C., Two new flavones from Eupatorium coelestinum. Phytochemistry (Elsevier) 1979, 18, (11), 1859-61.

(254) Reddy, R. V. N.; Reddy, B. A. K.; Gunasekar, D., A new acylated flavone glycoside from Colebrookea oppositifolia. J. Asian Nat. Prod. Res. 2009, 11, (2), 183-186. 